\title{
Investigating the Role of Trichodesmium spp. in the Oceanic Nitrogen Cycle through Observations and Models \\ by \\ Elise Marie Black Olson
}

B.S. in Earth, Atmospheric, and Planetary Sciences, Massachusetts

Institute of Technology (2006)

M.S. in Climate Physics and Chemistry, Massachusetts Institute of

Technology (2006)

Submitted in partial fulfillment of the

requirements for the degree of

Doctor of Philosophy in Physical Oceanography

at the

MASSACHUSETTS INSTITUTE OF TECHNOLOGY

and the

WOODS HOLE OCEANOGRAPHIC INSTITUTION

June 2014

(C) Elise Marie Black Olson, 2014. All rights reserved.

The author hereby grants to MIT and to WHOI permission to reproduce and distribute publicly paper and electronic copies of this thesis document in whole or in part.

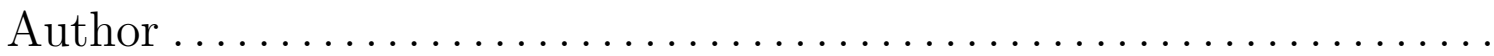

Joint Program in Physical Oceanography

Massachusetts Institute of Technology

Woods Hole Oceanographic Institution

May 1, 2014

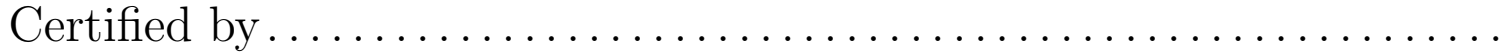

Dennis McGillicuddy

Senior Scientist

Thesis Supervisor

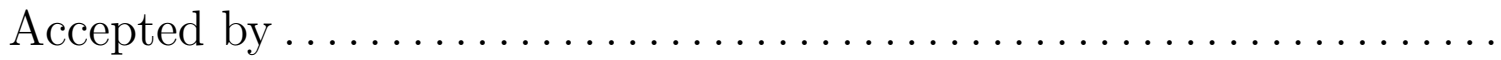

Glenn Flierl

Chair, Joint Committee for Physical Oceanography 


\title{
Investigating the Role of Trichodesmium spp. in the Oceanic Nitrogen Cycle through Observations and Models
}

by

\author{
Elise Marie Black Olson
}

\author{
Submitted to the Joint Program in Physical Oceanography - Massachusetts \\ Institute of Technology / Woods Hole Oceanographic Institution \\ on May 1, 2014, in partial fulfillment of the \\ requirements for the degree of \\ Doctor of Philosophy in Physical Oceanography
}

\begin{abstract}
This work concerns the nitrogen fixation and abundance of Trichodesmium colonies in the western subtropical-tropical North Atlantic and their connections with physical processes. Data were collected in fall 2010 and spring 2011, primarily using the Video Plankton Recorder (VPR). A data processing procedure for estimating the abundance of rare taxa was devised to take advantage of the accuracy of manual classification and the effort savings of automatic classification. The procedure entails selecting a subset of the original dataset, classifying it with automated software, and then manually correcting each classification. The method was validated through comparisons with fully classified VPR data and with abundance data based on microscopic enumeration on preserved samples.

Correlations of Trichodesmium colony abundance with the eddy field emerged in two subsets of the VPR observations. In fall 2010, local maxima in abundance were observed in a series of cyclones. We hypothesized Ekman transport convergence/divergence in cyclones/anticyclones as a driving mechanism. We investigated the process using idealized three-dimensional models of buoyant colonies in eddies. Elevated abundances in anticyclones in spring 2011 were correlated with anomalously fresh water, suggesting riverine input as a driver of the relationship.

Finally, we evaluated the hypothesis of Davis and McGillicuddy (2006) that Trichodesmium nitrogen fixation in the North Atlantic may be underestimated by conventional sampling methods, based on their VPR observations of higher than expected colony abundances at depth in the subtropical North Atlantic. A bio-optical model was developed based on carbon-normalized nitrogen fixation rates measured in fall 2010 and spring 2011 and used to estimate nitrogen fixation over the VPR transects. Estimates of abundance and nitrogen fixation were similar in magnitude and vertical and geographical distribution to estimates compiled in a database by Luo et al. (2012). In the mean, VPR-based estimates of volume-specific nitrogen fixation
\end{abstract}


rates at depth in the tropical North Atlantic were not inconsistent with estimates derived from conventional sampling methods. Based on this analysis, if Trichodesmium nitrogen fixation is underestimated, it is unlikely that it is attributable to underestimation of deep colony abundances due to mechanical disturbance during net-based sampling.

Thesis Supervisor: Dennis McGillicuddy Title: Senior Scientist 


\section{Acknowledgments}

First I would like to thank my advisor, Dr. Dennis McGillicuddy. His conscientious and ethical approach to all aspects of his work inspires confidence. I have benefitted from his insightful critiques and comments, generosity with his time, and high expectations.

My thesis committee, Dr. Cabell Davis, Dr. Sonya Dyhrman, Dr. Glenn Flierl, and Dr. John Waterbury, and defense chair, Dr Phoebe Lam, all enthusiastically provided very helpful advice and criticism which led to improvements in the quality of this work. Dr. Sallie (Penny) Chisholm also provided guidance in the early stages of this work. I value each of their insights and expertise.

This work would not have been possible without the dedicated efforts of the crew, officers, and science participants of the cruises OC469 and OC471 on the R/V Oceanus. Dennis McGillicuddy, Sonya Dyhrman, Cabell Davis, and John Waterbury were responsible for the planning and execution of the field work. Abby Heithoff carried out nitrogen fixation assays and participated in many other aspects of the data collection and subsequent analysis. Additional contributions were made at sea and on land by Dr. Valery Kosnyrev, Olga Kosnyreva, Dr. Larry Anderson, Keston Smith, Nancy Copley, Michael Oates, Dr. Mònica Rouco Molina, Louie Wurch, and Hannah Joy-Warren. Special thanks to Larry Anderson for teaching me to use POP, Valery Kosnyrev for the processing of sea level anomaly data, and Dr. Andy Solow for advice on statistics.

I would like to thank everyone who has worked in the Academic Programs Office at WHOI during my time here, especially Julia Westwater, Tricia Gebbie, Christine Charette, Marsha Gomes, Valerie Caron, and Lea Fraser, as well as Ronni Schwartz at MIT. They are an incredibly competent, knowledgeable, compassionate, and helpful group of people who make the Joint Program run smoothly. When a student in the Joint Program has a problem or question, they are often the first people we turn to.

Thank you to Dr. Mike Neubert, Dr. Hal Caswell, and the rest of the mathematical ecology group at WHOI for allowing me to participate early on in my time at WHOI in their meetings and retreat, which were the setting for many thoughtprovoking and interesting scientific discussions.

I feel incredibly lucky to have been a part of the WHOI community. Everyone I have interacted with here has demonstrated incredible skill and dedication to their jobs as well as commitment to creating a supportive work environment. To my classmates and officemates, especially Ping, Li Ling, Abby, Holly, Rebecca, Wilken, Sophie, Rachel, Alec, and Marilena, thank you for all the interesting discussions on work and life. I've learned a lot from all of you. Thank you to friends in Woods Hole and elsewhere for the support and happy memories, and especially to Li Ling, Alec, Liz, and Taylor for their support in the past few months as I've been finishing up this work.

Paty Matrai and Barney Balch, my bosses at the Bigelow Laboratory for Ocean Sciences before I started my PhD, have been excellent mentors since I first met them, 
serendipitously, at Spanish Speaking Night at the Second Congregational Church in Newcastle, Maine.

Finally, I would like to thank my family. My uncle, Chris Black, is always there for me. My parents, Jane Black and Greg Olson, have always been my greatest supporters. They cheer me on when things are going well and cheer me up when they aren't. My Dad inspired me to pursue a career in science and my Mom made me believe I could do it. Thank you both.

This work was supported through a NASA Earth and Space Science Fellowship (NASA NNX11AL59H Understanding the role of the nitrogen-fixing cyanobacterium Trichodesmium in the oceanic nitrogen and carbon cycles: in situ measurement, satellite observation, and biogeochemical modeling) as well as fellowship support from the Ocean Life Institute and Academic Programs Office at WHOI. Additional grant support was provided by NSF OCE-0925284 Quantification of Trichodesmium spp. Vertical and Horizontal Abundance Patterns and Nitrogen Fixation in the Western North Atlantic, NSF OCE-1048897 MOBY: Modeling Ocean Variability and Biogeochemical Cycles, NASA NNX13AE47G Physical and Biological Dynamics of Nonlinear Mesoscale Eddies: Satellite Observations, in situ Measurements, and Numerical Simulations on a Global Scale, and NASA NNX08AL71G Carbon cycling in the North Atlantic from regional to basin scales: satellite data, in situ observations, and numerical models. 


\section{Contents}

1 Introduction $\quad 11$

1.1 Biogeography and Abundance . . . . . . . . . . . . . . . 13

1.2 Trichodesmium Nitrogen Fixation and Its Fate . . . . . . . . . . . 15

1.3 Thesis Outline . . . . . . . . . . . . . . . 16

2 VPR Data Processing Methods and Verification 21

2.1 Abstract . . . . . . . . . . . . . . . . . 21

2.2 Introduction . . . . . . . . . . . . . . . . . 22

2.3 Methods . . . . . . . . . . . . . . . . . . . . 23

2.3.1 Data Collection .................. 23

2.3.2 VPR Data Processing . . . . . . . . . . . . . . 27

2.3.3 Comparison of Abundances from VPR and Microscopic Enumeration ...................... 37

2.4 Results and Discussion . . . . . . . . . . . . . . . . . 37

2.4.1 Comparison of Subsampled with Fully Classified Data . . . . 37

2.4.2 Comparison of Abundance Estimates: VPR vs. Microscopic

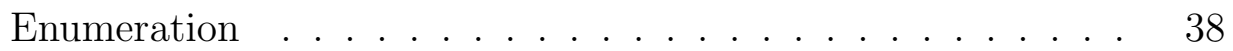

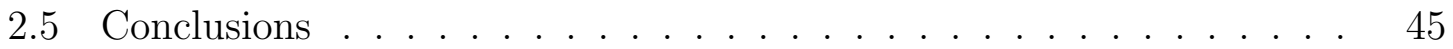

3 Mesoscale Eddies and Trichodesmium Abundance 49

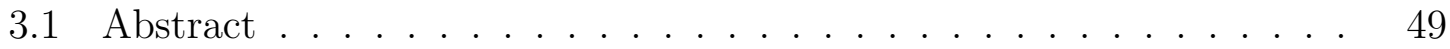

3.2 Introduction . . . . . . . . . . . . . . . . 50 
3.3 Methods . . . . . . . . . . . . . . . . . . . . . 54

3.3.1 Observational Methods . . . . . . . . . . . . . . . . . 54

$3.3 .2 \quad$ Model . . . . . . . . . . . . . . . . . . . . . . . . 58

3.4 Results . . . . . . . . . . . . . . . . . . . . . . . 61

3.4.1 Fall 2010: Enhanced Abundance of Trichodesmium Colonies in Cyclones . . . . . . . . . . . . . . . . . . . 62

3.4 .2 Conceptual Model . . . . . . . . . . . . . . . . . . 65

3.4.3 Numerical Model . . . . . . . . . . . . . . . . . . . . . . . . . 69

3.4.4 Spring 2011: Trichodesmium Colonies in Anticyclones . . . . . 76

3.5 Discussion . . . . . . . . . . . . . . . . . . . . . . . 83

3.5.1 Fall 2011: Trichodesmium Colonies in Cyclones . . . . . . . 83

3.5.2 Spring 2011: Trichodesmium Colonies in Anticyclones . . . . . 86

3.6 Conclusions . . . . . . . . . . . . . . . . . . . . . . . . . . . . . . 88

4 How much do deep populations contribute to nitrogen fixation by Trichodesmium spp. in the tropical-subtropical North Atlantic? 91

4.1 Abstract . . . . . . . . . . . . . . . . . . 92

4.2 Introduction . . . . . . . . . . . . . . . . . . . 92

4.3 Methods . . . . . . . . . . . . . . . . . . 94

4.3.1 Data Collection . . . . . . . . . . . . . . . . . 94

4.3.2 Data Analysis and Modeling . . . . . . . . . . . . . . . 101

4.4 Results and Discussion . . . . . . . . . . . . . . . . 107

4.4 .1 PAR Estimation . . . . . . . . . . . . . . 107

4.4.2 Relationship of Nitrogen Fixation with Temperature . . . . 110

4.4 .3 Nitrogen Fixation and Light . . . . . . . . . . . . . . . 111

4.4.4 Carbon Per Colony . . . . . . . . . . . . . . . . . . . 116

4.4 .5 Model Assessment . . . . . . . . . . . . . . . . . 117

4.4.6 Mesoscale to Sub-Basin-Scale Estimates of

Trichodesmium Biomass and Nitrogen Fixation . . . . . . 121 
4.4.7 Large-scale Spatial Variations in Nitrogen Fixation:

Comparisons with Luo et al. (2012) and Davis and McGillicuddy

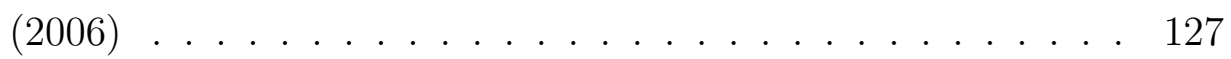

4.5 Conclusions . . . . . . . . . . . . . . . . . . 140

5 Conclusions $\quad 145$

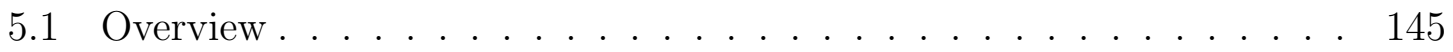

5.2 Future Directions . . . . . . . . . . . . . . . . . 147

5.3 Summary and Scientific Impact . . . . . . . . . . . . . . . . . . 149

A Nitrogen Fixation Method Comparison

153 


\section{Chapter 1}

\section{Introduction}

Trichodesmium is a genus of nitrogen-fixing nonheterocystous colonial cyanobacteria most abundant in warm tropical waters. Macroscopic colonies at times form surface aggregations visible from ships, referred to colloquially as "sea sawdust" (Capone et al., 1997). It exists in the forms of colonies and thread-like free filaments, or trichomes, themselves made up of many cells. Colonies are groupings of trichomes arranged in characteristic morphologies. The most common are rafts (or tufts), puffs, and bowties. Morphology has not been found to be a good indicator of species, with the same species exhibiting multiple morphologies. Two of the most common species are T. erythraeum and T. thiebautii. Colonies often contain many other microorganisms living in close association with Trichodesmium (Hewson et al., 2009).

Trichodesmium is unusual among nonheterocystous cyanobacteria in that it fixes nitrogen concurrently with photosynthesis. The nitrogenase enzyme responsible for nitrogen fixation is inactivated by oxygen, which is evolved during photosynthesis. Trichodesmium fixes nitrogen only in the presence of light (Wilson et al., 2012) with modulation over the course of the light period roughly consistent with variation in solar irradiance (Saino and Hattori, 1978). One way in which Trichodesmium is thought to mitigate the adverse effects of oxygen accumulation on the machinery of nitrogen fixation is through tight coupling between photosynthetic oxygen evolution 
and oxygen uptake by other processes such as the Mehler reaction (Berman-Frank et al., 2007). Other suggested mechanisms by which Trichodesmium may avoid oxygen damage to nitrogenase include temporal segregation during the photoperiod and spatial segregation within the cell (Berman-Frank et al., 2001).

The diel pattern in nitrogen fixation is at least in part controlled by a circadian rhythm (Chen et al., 1996). Although short-term changes in light conditions have been demonstrated to significantly impact nitrogenase activity, they have a comparatively small impact on transcription of genes associated with nitrogenase (Chen et al., 1999). On longer timescales, physiological adaptations to low irradiance have been observed in Trichodesmium in culture, including increased cell diameter for light absorption and changes in coupling of phycobilisomes with the photochemical reaction centers (Andresen et al., 2010).

Trichodesmium colonies are typically most abundant in surface or near-surface waters due to their buoyancy. Increased abundance is often associated with stratification (Post et al., 2002; Capone et al., 1997). Vertical distributions are thought to be determined largely through balance between buoyancy and wind-driven mixing (Capone et al., 1997). In the Red Sea, however, vertical organization by colony morphology, with rafts dominant near the surface and puffs in the lower half of the euphotic zone, was observed over a period of two months (Post et al., 2002). This was interpreted as suggesting that the coexisting colony morphologies may occupy different niches.

Walsby (1978) measured floating velocities in field-collected sampled and found that a majority were positively buoyant. In the same study, colonies were subjected to increasing pressure to the point of gas vesicle collapse, at which point they sank, indicating that the gas vacuoles play an important role in providing colony buoyancy. Subsequent studies indicate that variations in colony buoyancy are induced through changes in cellular carbohydrate:protein ratio, with carbohydrate levels increasing during the light period as photosynthesis occurs (Villareal and Carpenter, 2003). The potential of this type of buoyancy modulation to facilitate vertical migra- 
tion, allowing colonies to acquire phosphorus at depth and return to the surface for light, has been investigated through observations (Letelier and Karl, 1998) and mechanistic models (White et al., 2006). The dominant pattern simulated by the White et al. (2006) model involved trapping of colonies at the surface, while the next most common solution involved mining to the average depth of the phosphocline. Such migratory behavior could result in spatial decoupling of nitrogen and phosphorus cycling (Letelier and Karl, 1998).

\subsection{Biogeography and Abundance}

Typical doubling times for Trichodesmium are on the order of 1-5 days (Carpenter et al., 1993; Rodier and Borgne, 2010; Holl and Montoya, 2008). At these comparatively slow growth rates, Trichodesmium can outcompete other faster-growing organisms only when growth of those organisms is nutrient-limited. Thus, Trichodesmium thrives globally in nitrogen-poor oligotrophic tropical and subtropical waters. Its range is also temperature-dependent, with high concentrations, for instance, in the warm waters of the western Atlantic (Goebel et al., 2010). A prime environment for Trichodesmium growth, the oligotrophic waters of the subtropical-tropical North Atlantic also have the greatest data coverage with respect to measurements of Trichodesmium nitrogen fixation and abundance and diazotrophic activity in general (Luo et al., 2012).

With its ability to fix nitrogen, Trichodesmium growth is typically limited by the availability of iron and phosphorus. A strong competitor for phosphorus, Trichodesmium is able to use forms of dissolved organic phosphorus (DOP), such as phosphonate, that are unavailable to many other organisims (Dyhrman et al., 2006). Mulholland et al. (2002) found that alkaline phosphorus activity, likely an indicator of phosphorus scavenging, decreased in the presence of dissolved inorganic phosphorus (DIP) and increased in the presence of DOP. In culture, Trichodesmium grew equally well when supplied with DIP or DOP. In the Sargasso Sea, Orchard et al. 
(2010) showed that Trichodesmium is a better competitor for DOP than for dissolved inorganic phosphorus (DIP) based on uptake rates.

In the Atlantic, Trichodesmium is thought to be generally phosphorus limited. Iron is supplied to this region more regularly than to the Pacific due to Saharan dust deposition and riverine inputs (Mahowald et al., 2005). Trichodesmium abundances increase from the subtropical to tropical North Atlantic (Taboada et al., 2010). Typically Trichodesmium abundances are high in the tropical Atlantic from $5 \mathrm{~S}$ to $15 \mathrm{~N}$ but nearly absent from the South Atlantic gyre (Tyrrell et al., 2003; Fernández et al., 2010). Trichodesmium abundance has been connected with riverine nutrient inputs in regions influenced by both the Amazon and Congo rivers (Coles et al., 2004; Goebel et al., 2010; Subramaniam et al., 2008; Foster et al., 2009). Sañudo-Wilhelmy et al. (2001) observed in the central Atlantic that nitrogen fixation rates were independent of dissolved iron levels, whether within colonies or in the environment, instead correlating with cellular phosphorus levels. Comparisons have shown greater phosphorus stress in Trichodesmium populations in the North Atlantic than in the Pacific based on maximum phosphate uptake rates (Sohm et al., 2008) and endogenous alkaline phosphatase activity (Hynes et al., 2009).

In addition to evidence of phosphorus limitation, correspondence has been observed between iron deposition events and Trichodesmium growth in the North Atlantic. Trichodesmium nitrogen fixation varied with seasonal dust deposition at BATS (Orcutt et al., 2001). On the West Florida shelf, a 1999 Saharan dust event preceded a Trichodesmium bloom involving a 100-fold increase in biomass and drawdown of both DOP and DIP below the limit of detection (Lenes and Heil, 2010). Mahaffey et al. (2003) observed biogeochemical signatures of nitrogen fixation along with cyanobacteria pigments following a dust deposition event predicted by model in the eastern subtropical North Atlantic.

Ecosystem modeling studies suggest that the primary factors controlling the distribution of nitrogen fixers are nutrient concentrations. In a self-organizing phytoplankton community global model, nitrogen fixation was favored where iron and 
phosphorus were available but fixed nitrogen was limiting (Monteiro et al., 2011). Such trait-based models are able to reproduce observed large-scale diazotroph distributions, with Trichodesmium analogs the dominant diazotrophs in the North and tropical Atlantic and the Arabian Sea where phosphorus was limiting but unicellular diazotrophs dominant in the iron-limited South Atlantic, Pacific, and Indian Oceans (Monteiro et al., 2010). A modeling analysis of nitrogen, iron, and phosphorus resource competition suggested that iron played the dominant role in determining diazotroph distributions with secondary influence by phosphorus (Ward et al., 2013).

\section{$1.2 \quad$ Trichodesmium Nitrogen Fixation and Its Fate}

Estimates of Trichodesmium nitrogen fixation suggest that it is in some regions as large a source of nitrogen to the euphotic zone as diapycnal mixing (Capone et al., 2005). Based on colony abundance over a period of three years, one study estimated Trichodesmium nitrogen input at $5 \mathrm{mmol} \mathrm{N} \mathrm{m}^{-2}$ year $^{-1}$ at BATS (Orcutt et al., 2001). Another two-year study at BATS estimated Trichodesmium nitrogen fixation rates as high as $92 \mathrm{mmol} \mathrm{N} \mathrm{m}^{-2}$ year ${ }^{-1}$, nearly three times the contribution by unicellular cyanobacteria Groups A and B of $36 \mathrm{mmol} \mathrm{N} \mathrm{m}^{-2}$ year ${ }^{-1}$ (Goebel et al., 2007). Trichodesmium has been estimated to input a minimum of $1.6 \times 10 \times 10^{12}$ mol N year $^{-1}$ to the tropical North Atlantic (Capone et al., 2005). Nitrogenase activity has been observed to be higher in T. thiebautii than T. erythraeum (Carpenter et al., 1993), and in colonies compared to free filaments (Letelier and Karl, 1998).

In one study in the Gulf of Mexico, the ratio of net $\mathrm{N}_{2}$ uptake to gross $\mathrm{N}_{2}$ fixation ranged from $19 \%$ to $91 \%$, suggesting an excretion rate of fixed nitrogen from cells ranging from $9 \%$ to $81 \%$ with an average of $52 \%$ (Mulholland et al., 2006). Additional dissolved nitrogen may become available through remineralization as Trichodesmium blooms decline (Mulholland et al., 2006). Trichodesmium blooms may provide the bioavailable nitrogen required to fuel blooms of Karenia brevis (Mulholland et al., 2006; Lenes and Heil, 2010) and Gymnodinium breve (Lenes and Heil, 2010) in the 
Gulf of Mexico. Additionally, co-occurance of Trichodesmium with diatoms in the Kuroshio current has been interpreted as suggesting transfer of fixed nitrogen from Trichodesmium to diatoms, potentially associated with more efficient export of carbon and nitrogen (Chen et al., 2011). Letelier and Karl (1996) observed increased autotrophic carbon beyond what they attributed to Trichodesmium biomass during a bloom at station ALOHA and attributed it to release of fixed nitrogen by Trichodesmium .

Grazing on Trichodesmium by copepods and other zooplankton has been a topic of contention. Hawser et al. (1992) studied the effects of Trichodesmium on grazers and found that T. thiebautii exhibited greater toxicity than T. erythraeum to calanoid and cyclopoid copepods, whereas harpacticoid copepods were unaffected. In the Bahamas and Caribbean, shipboard experiments demonstrated incorporation of Trichodesmium carbon into copepod (Macrosetella gracilis) biomass (O'Neil, 1998). However, Eberl and Carpenter (2007) found no Trichodesmium in the gut contents of the harpacticoid copepod M. gracilis in the North Pacific gyre and concluded that while Trichodesmium may form a substrate for juvenile development it was not a food source. Other sources of Trichodesmium mortality include programmed cell death in response to iron and phosphorus starvation (Berman-Frank et al., 2004) and viral lysis (Hewson et al., 2004).

\subsection{Thesis Outline}

The balance (or imbalance) of the marine nitrogen cycle has been subject to debate. During the 1980s, disparities were identified between new production estimates based on oxygen utilization rates and lower estimates based on the combination of net

primary production measured by ${ }^{14} \mathrm{C}$ incubation with estimated recycling efficiencies within the euphotic zone in the Sargasso Sea (Jenkins and Goldman, 1985). Since new production is defined as primary production utilizing nitrogen newly supplied to the euphotic zone through upwelling, atmospheric deposition, and biological fixa- 
tion, this imbalance reflected uncertainty in the magnitude of fixed nitrogen supply to the region. More recently, assessments of the summed sources and sinks of fixed nitrogen to the global oceans have produced varying results, primarily attributable to differing estimates of water column and benthic denitrification rates (Gruber, 2008). These estimates range from near balance (Gruber, 2004, 2008) to modest (Galloway et al., 2004) or large, $200 \mathrm{Tg} \mathrm{Nyr}^{-1}$ (Codispoti et al., 2001), imbalance. As a point of reference, total global nitrogen fixation is estimated between 100 and $150 \mathrm{Tg} \mathrm{N} \mathrm{yr}-1$ (Gruber, 2008). A possibility discussed by Codispoti et al. (2001), Galloway et al. (2004), and Gruber (2008) is that some fraction of the apparent imbalance could be real and the result of anthropogenic perturbation; however, Deutsch et al. (2007) argue against such a true imbalance on the basis of tight coupling between fixation and denitrification. Other means of resolving the apparent imbalance are downward revision of denitrification estimates or upward revision of estimates of fixed nitrogen sources, such as nitrogen fixation. The uncertainty associated with each of these elements is large.

Here we focus on a single component of the marine nitrogen budget, nitrogen fixation by Trichodesmium. Since nitrogen often limits primary production, nitrogen made available through fixation by Trichodesmium contributes to primary production and the biological pump, and potentially to carbon export from the surface waters. Thus, in addition to further constraining the marine nitrogen budget, greater understanding of the dynamics of nitrogen fixation by Trichodesmium will contribute to quantification of the global carbon cycle.

Davis and McGillicuddy (2006) reported two intriguing findings based on a video plankton recorder (VPR) survey across the subtropical North Atlantic. The first finding was unexpectedly uniform distributions of Trichodesmium colonies with depth over much of the survey region in contrast to the canonical Trichodesmium colony profile in which abundance decays rapidly with depth. Potentially, this canonical profile could be skewed by the use of invasive sampling techniques, such as net tows, due to the fragility of Trichodesmium colonies. Such methods might preferentially de- 
stroy deep colonies having longer transit durations between the sampling depth and the surface. The second finding was a correlation between Trichodesmium colony abundance and anticyclonic eddies. Improved understanding of the drivers of spatial and temporal variability of patchy Trichodesmium colony distributions could be leveraged to further constrain estimates of the contribution of Trichodesmium to nitrogen fixation. However, the Davis and McGillicuddy (2006) study was conducted in a region of relatively low Trichodesmium abundance. Would VPR sampling reveal these same patterns in the high-abundance tropical North Atlantic?

In this study, we address this question in the context of the overarching question, what is the contribution of nitrogen fixation by Trichodesmium to the global nitrogen cycle? This problem is approached through exploration of a series of more specific and tractable questions:

- Is Trichodesmium more abundant at depth than previously believed in the southwestern North Atlantic?

- Where deep Trichodesmium populations are present, how much nitrogen do they fix?

- What environmental factors influence spatial patterns in Trichodesmium abundance?

- Is Trichodesmium abundance higher in anticyclonic eddies in the southwestern North Atlantic?

These questions are addressed through analysis of data from a series of VPR transects and targeted station work including nitrogen fixation assays carried out in the western tropical North Atlantic in October 2010 and April-May 2011 aboard the $\mathrm{R} / \mathrm{V}$ Oceanus. In Chapter 2 we discuss novel methods employed in the extraction of Trichodesmium colony abundance from the VPR dataset. In Chapter 3, we elucidate some of the environmental factors influencing the distribution of Trichodesmium colonies at the mesoscale. In Chapter 4, we develop a bio-optical model and use it 
to estimate nitrogen fixation on the VPR transects. We compare the magnitude and depth-distribution of Trichodesmium abundance and modeled nitrogen fixation rates with measurements recently compiled in a global database. Finally, in Chapter 5, we synthesize all findings and discuss their implications for the role of Trichodesmium in the nitrogen cycle in the tropical-subtropical North Atlantic. 


\section{Chapter 2}

\section{VPR Data Processing Methods and Verification}

\subsection{Abstract}

At currently achievable levels of accuracy, automatic classification of rare taxa (10\% or less of total population) can produce abundance estimates with levels of error comparable to true abundance. One way to achieve greater accuracy is through manual correction of classified images. However, this is impractical for very large datasets. A method has been devised to extract abundance of rare taxa from large VPR datasets with reduced manual classification effort. Rather than classifying the full dataset, a subset of saved images are selected for classification. Abundances are calculated based on this subset. The sample volume and number of identifications are estimated on a grid, with the sample volume in each cell adjusted to account for the fraction of total images included in the subset. By reducing the size of the sample used to estimate abundance, some accuracy is lost through the introduction of random error. However, this tendency is partly counteracted through increased grid size, resulting in a larger number of images per grid cell. Abundances estimated based on full and subsample datasets are compared at multiple grid resolutions. At grid cell dimensions of $33 \mathrm{~km}$ horizontally by $5 \mathrm{~m}$ vertically, the variation coefficients of 
the root mean square error were $1.01,1.31$, and 1.36 for the three rare taxa analyzed. For a single bin covering the same $165 \mathrm{~km}$ by $150 \mathrm{~m}$ area, the variation coefficients of the RMSE decreased to 0.003, 0.093, and 0.004. Additionally, VPR abundance estimates based on full and subsample datasets are compared to nearby abundance estimates obtained through microscopic counts of filtered preserved samples. Results are consistent within anticipated levels of sample variability at low abundance levels but VPR-based estimates are biased low at high abundance levels.

\section{$2.2 \quad$ Introduction}

The VPR is a towed underwater digital microscope. It consists of a towed body, containing a synchronized video camera and xenon strobe as well as a CTD (Davis et al., 2005). Data are sent in real-time through a fiber-optic cable to a shipboard computer system. While the ship is underway, the VPR undulates between the surface (5-10 m) and 120-150 m depth, completing an up-down cycle approximately every six minutes. Since it is towed at 10 knots $(5.1 \mathrm{~m} / \mathrm{s})$, this corresponds to one vertical cycle every $1.8 \mathrm{~km}$. Navigation to starboard of the ship's wake near the surface, in addition to strategic camera and strobe placement and towfish design, minimize disturbance of the regions sampled. At a frame rate of $30 \mathrm{~Hz}$, each 984 x 1009 pixel video frame is passed through object-identification software to pick out "regions of interest" and save them, using a time-stamp naming convention, to a hard disk. In addition to imaging, the VPR records pressure, temperature, salinity, photosynthetically active radiation (PAR), oxygen, and fluorescence.

Automated classification software exists for the identification of VPR images. However, at currently achievable levels of accuracy, false positive identifications can obscure true abundance signals for rare taxa that make up a small fraction of the total number of images collected. Manual correction of automated output greatly improves accuracy but can be prohibitively time-consuming for large dataset. Here we develop a data processing procedure to decrease manual classification effort but 
increase accuracy. A subset of the original dataset is selected for classification with manual correction. Abundances are estimated based on this subset at coarser resolution than typical VPR abundance estimates to compensate for the decrease in sample size.

In the following sections, we discuss the application of this method to Trichodesmium colony abundance data collected in the western tropical-subtropical North Atlantic. Images were classified using a combination of image recognition software with human-mediated correction. An entire dataset from fall 2010 was classified and manually corrected. A subset from spring 2011 was processed as described above. An isolated segment of the spring 2011 data was fully classified to allow for comparison between the subsampled and fully sampled abundance estimates. Both the fully classified fall 2010 data and the subsampled spring 2011 data were compared with abundance estimates made by microscopic enumeration at nearby sampling stations in order to validate the accuracy of the two methodss.

\subsection{Methods}

\subsubsection{Data Collection}

Sampling occurred on two cruises on the $\mathrm{R} / \mathrm{V}$ Oceanus, the first from October 1-22, 2010, and the second from April 23-May 13, 2011. The planned cruise track consisted of a meridional section from Bermuda to Puerto Rico, followed by a southeastward leg to a longitude of approximately $40^{\circ} \mathrm{W}$ and a southwestward leg ending just east of Barbados. Precise sampling locations (Figures 2-1 and 2-2; Table 4.1) were chosen during the cruises as informed by satellite observations of sea surface height, real-time analysis of VPR Trichodesmium abundance observations, and ocean-colorbased estimates of Trichodesmium bloom probability (Westberry and Siegel, 2006; Westberry et al., 2005) provided by Toby Westberry. This adaptive strategy allowed sampling to be directed toward interesting physical and biological features such as eddies and areas of elevated Trichodesmium abundance. The VPR was towed between 


\begin{tabular}{|c|c|c|c|c|c|c|c|}
\hline \multirow[b]{2}{*}{ St. } & \multicolumn{3}{|c|}{$O C_{4} 69$} & \multicolumn{4}{|c|}{$O C_{4} 71$} \\
\hline & Lat. & Lon. & Date & St. & Lat. & Lon. & Date \\
\hline 2 & 31.687 & 64.105 & 05 Oct. 2010 & 1 & 31.668 & 64.166 & 26 Apr. 2011 \\
\hline 3 & 28.587 & 65.058 & 06 Oct. 2010 & 2 & 25.500 & 66.233 & 28 Apr. 2011 \\
\hline 4 & 28.969 & 65.988 & 07 Oct. 2010 & 3 & 22.181 & 65.939 & 29 Apr. 2011 \\
\hline 5 & 29.082 & 66.109 & 08 Oct. 2010 & 4 & 20.010 & 63.290 & 30 Apr. 2011 \\
\hline 6 & 25.512 & 67.253 & 10 Oct. 2010 & 5 & 17.928 & 60.953 & 01 May 2011 \\
\hline 7 & 22.491 & 67.303 & 11 Oct. 2010 & 6 & 18.226 & 61.297 & 02 May 2011 \\
\hline 8 & 21.266 & 64.003 & 12 Oct. 2010 & 7 & 15.529 & 59.783 & 03 May 2011 \\
\hline 9 & 17.254 & 58.505 & 14 Oct. 2010 & 8 & 14.945 & 57.869 & 04 May 2011 \\
\hline 10 & 15.227 & 54.659 & 15 Oct. 2010 & 9 & 15.146 & 57.492 & 05 May 2011 \\
\hline 11 & 13.491 & 51.309 & 16 Oct. 2010 & 10 & 12.353 & 56.341 & 06 May 2011 \\
\hline 12 & 11.915 & 48.459 & 17 Oct. 2010 & 11 & 13.940 & 54.550 & 07 May 2011 \\
\hline 13 & 11.537 & 51.357 & 18 Oct. 2010 & 12 & 15.777 & 52.754 & 08 May 2011 \\
\hline 14 & 12.209 & 54.520 & 19 Oct. 2010 & 13 & 15.626 & 53.032 & 09 May 2011 \\
\hline 15 & 12.896 & 58.594 & 20 Oct. 2010 & 14 & 15.587 & 53.010 & 10 May 2011 \\
\hline 15.1 & 12.563 & 56.663 & 21 Oct. 2010 & 15 & 16.463 & 55.440 & 11 May 2011 \\
\hline 16 & 12.547 & 56.340 & 21 Oct. 2010 & 16 & 14.741 & 57.455 & 12 May 2011 \\
\hline 17 & 12.565 & 56.622 & 21 Oct. 2010 & & & & \\
\hline
\end{tabular}

Table 2.1: Station locations and dates for both cruises. Latitudes are in degrees North and longitudes in degrees West.

stations (Figures 2-1 and 2-2). A series of cyclonic and anticyclonic eddy features were transected during each cruise.

\section{Discrete Water Sampling}

In addition to underway VPR tows, the sampling program included daily stations with associated nitrogen fixation experiments beginning at approximately 10:00 a.m. local time. At each station, water samples were collected with a rosette system in 10 L Niskin bottles at depths of 700, 500, 300, 200, 100, 80, 60, 40, $20 \mathrm{~m}$, and the surface. In the upper $80 \mathrm{~m}$, water samples were taken for the microscopic enumeration of Trichodesmium colonies and free trichomes by methods described by Carpenter et al. (2004). In brief, the samples were gravity filtered onto $10 \mu \mathrm{m} 47 \mathrm{~mm}$ filters through in-line filter holders attached to the spigot of the Niskin bottles. After filtration the filters were fixed for 30 minutes in the filter holders with $4 \%$ paraformaldehyde in 


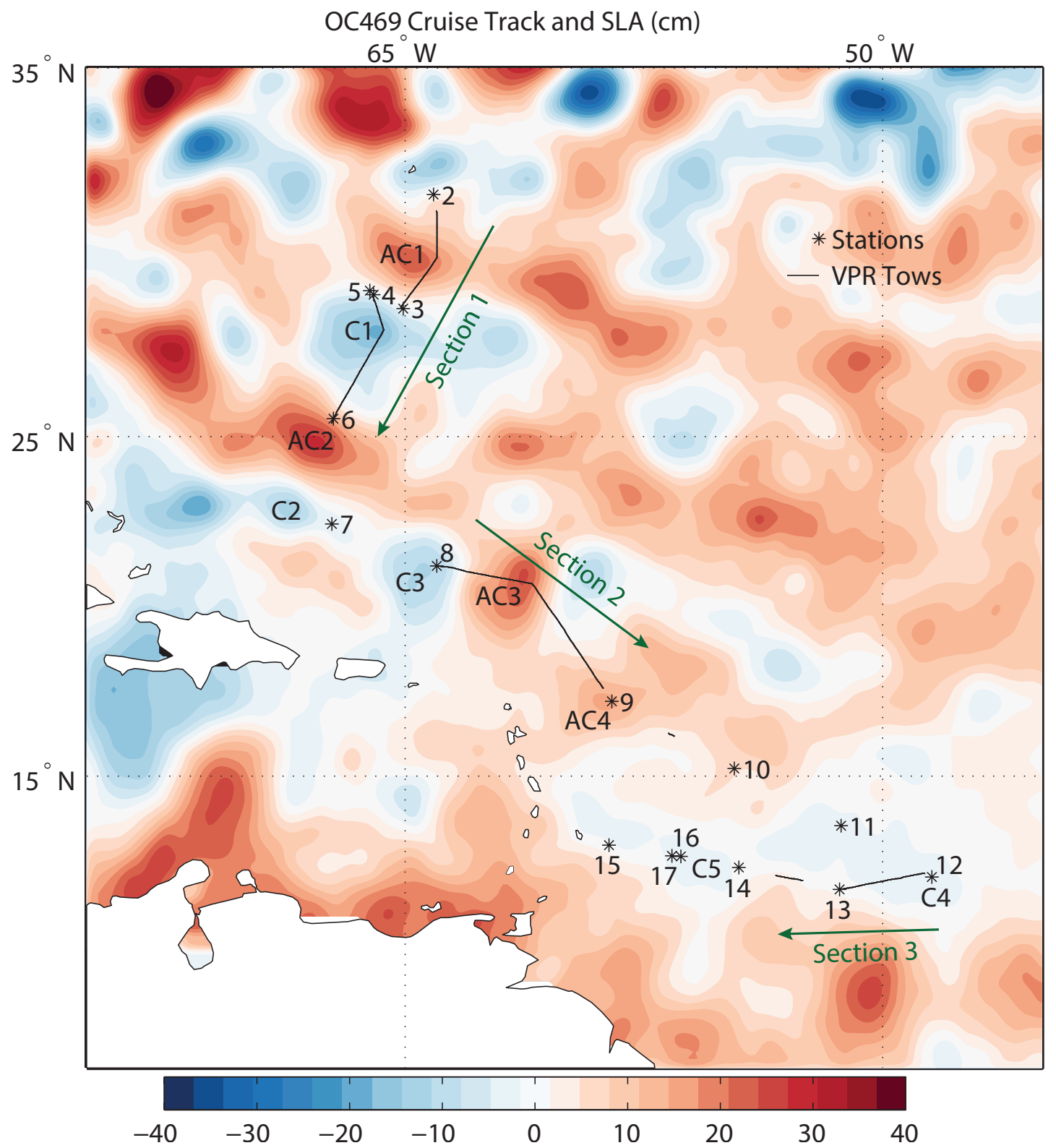

Figure 2-1: Locations of stations and VPR tows for cruise OC469 with sea level anomaly. Black lines indicate VPR tows. Stars indicate station locations. Targeted cyclones and anticyclones are labeled with prefixes "C" and "AC", respectively. Labeled sections are referred to in subsequent analysis. 


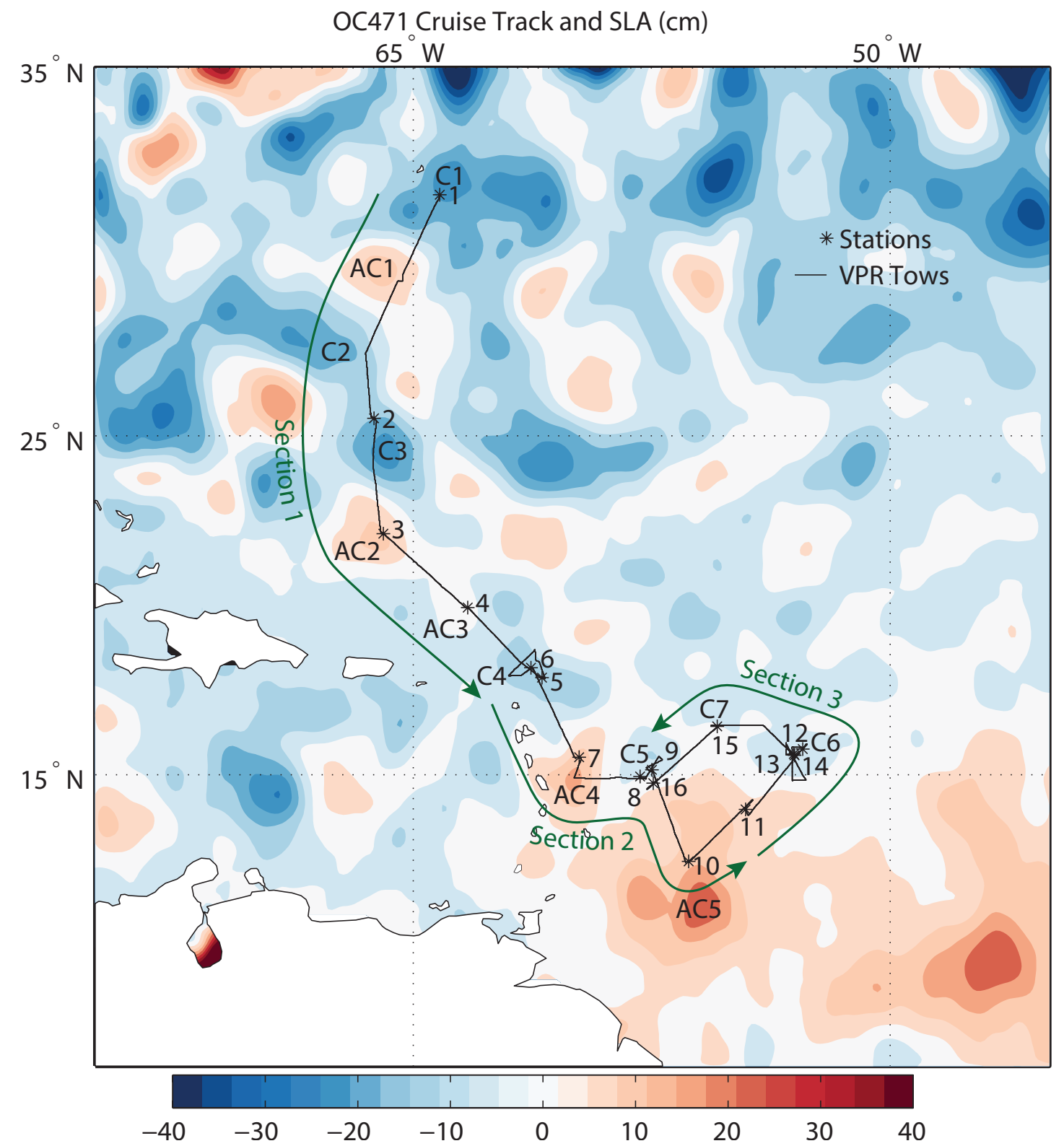

Figure 2-2: Locations of stations and VPR tows for cruise OC471 with sea level anomaly. Black lines indicate VPR tows. Stars indicate station locations. Targeted cyclones and anticyclones are labeled with prefixes "C" and "AC", respectively. Labeled sections are referred to in subsequent analysis. 
$0.2 \mu \mathrm{m}$ filtered sea water. Paraformaldehyde was removed and the filters were then mounted in immersion oil on slides with coverslips. The fixed preparations were stored frozen prior to enumeration. Trichodesmium spp. colonies and filaments (trichomes) were identified and counted with epifluorescence microscopy of phycoerythrin autofluorescence by imaging the entire filter. Colonies were defined as associations of 8 or more filaments. Colonies and filaments were enumerated in 70 samples from 14 stations from OC469. From OC471, colonies were enumerated in 43 samples from 9 stations, and filaments were enumerated in 39 samples from the same 9 stations.

\subsubsection{VPR Data Processing}

Once individual "regions of interest" (ROIs) containing separate objects are isolated from VPR frames and saved, they must be classified in order to extract abundance data. Automated software for the VPR system exists for this purpose $(\mathrm{Hu}$ and Davis, 2006). The most sophisticated version is a dual classification system (Hu and Davis, 2006) combining a learning vector quantization neural network (LVQ-NN) classifier using shape-based features and a support vector machine (SVM) classifier using texture-based features. Both classifiers must be trained by manually sorting an initial set of images and then extracting classification feature data from the sorted images. The LVQ-NN classifier fits a set of "neurons", or points in feature space, to the training data, and classifies images by associating them with the nearest neuron. The SVM classifier maps features to a higher-dimensional space that is separated into distinct regions associated with taxonomic groups. In the dual classification system, unless both classifiers agree on a given image, the classification is rejected, reducing the occurrence of false positives. A constant correction factor is applied to each category to correct for constant, systematic classifier error. The dual classification method has the advantage of reduced error compared to a single classifier while avoiding human mediation of the classification process, facilitating processing of large numbers of images.

Classifiers were trained for both cruises including the categories of interest for 


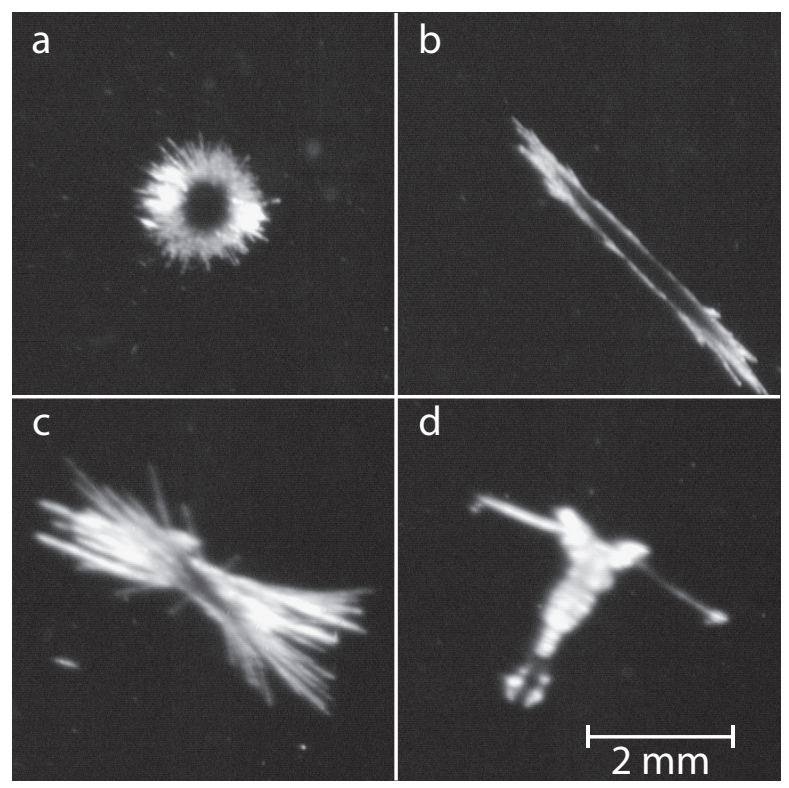

Figure 2-3: Sample VPR images from classification categories. a) Puff Trichodesmium colony morphology. b) Raft Trichodesmium colony morphology. c) Bowtie Trichodesmium colony morphology. d) Copepod. Scale shown is for the center of field of focus; however, the actual scale depends on the depth of each object within the field of view. 
this work - puff, raft, and bowtie Trichodesmium colony morphologies - as well as copepods and several other of the most common types. Samples of VPR images from Trichodesmium colony and copepod categories are shown in Figure 2-3.

In this data set, the target organism, Trichodesmium, constituted a small fraction of the total number of images. Most of the time, even in areas of relatively high Trichodesmium abundance, less than ten percent of all images were Trichodesmium. Given the low relative abundance of Trichodesmium colonies and typical accuracy levels, false positive identifications of other organisms can obscure the true Trichodesmium colony abundance patterns.

Consider the following situation. The classifier described by Hu and Davis (2006) accurately classified approximately $62 \%$ of images, overall. Assuming an accuracy level only slightly lower, at $60 \%$, a Trichodesmium fraction of $10 \%$ of all images, and that errors are evenly distributed across the categories, the expected number of true positive Trichodesmium identifications would be

$$
T P=0.6 \times 0.1 \times P=0.06 P,
$$

where $P$ is the total number of images. The expected number of false positive Trichodesmium identifications (images identified by the software as Trichodesmium that were not actually Trichodesmium ) for a seven-category classifier would be equal to the fraction of the non-Trichodesmium population that will be incorrectly classified, divided evenly over the number of categories available for misclassification:

$$
F P=\frac{(1-0.6) \times(1-0.1) P}{7-1}=0.06 P .
$$

Thus, the number of false positive identifications could easily be comparable to the number of true positive identifications.

Hu and Davis (2006) developed a correction method to account for this type of systematic misclassification. Calculated abundances are corrected by scaling by the ratio of the probability of detection for a given taxa (correct identifications/actual 
number in sample) to the specificity, or the probability that an identification of that taxa is correct (true identifications/(true + false identifications)).

However, Hu and Davis (2006) warned that the correction method is dependent on the consistency of the classification statistics, specifically the probability of detection and specificity for each taxa, over the study area. In particular, they note that the specificity for a given taxon covaries with its relative abundance. Here, we have set out to investigate patterns of Trichodesmium abundance across a broad array of physical and ecological conditions. It is therefore expected that the relative abundance, and hence specificity, of taxa will vary over the course of the cruise.

In fact, the level of accuracy achieved through automatic classification proved insufficient. The most serious barrier to automated classification was the fact that the two most abundant Trichodesmium colony morphologies, puffs and rafts, were not easily distinguished by available classification methods from other common image types. In particular, puff images were often confused with images of other circular shapes, such as unfocused images, some marine snow aggregations, Acantharia, and starfish larvae. Rafts were confused with many rod-shaped objects, including diatom chains. Indeed, manual classification of a one-hour segment of data, taken from a region of relatively high Trichodesmium abundance from the fall cruise, revealed that of 729 total Trichodesmium colony identifications by a computer classifier, 442 were true and 287 were false. Classification of an hour's data taken from a low Trichodesmiumabundance region by the same classifier yielded only 31 true positive identifications out of a total of 540 positive identifications. At these accuracy levels, variations in abundance of other image types could mask the Trichodesmium abundance signal.

In order to improve the accuracy of the VPR abundance data, manual intervention in the classification processes was deemed necessary. For the fall 2010 data, the entire data set was classified using the SVM classifier (although the dual classifier could have served the same purpose). Then, each image in each category was individually inspected and re-classified as necessary so that the raft, puff, and bowtie Trichodesmium colony morphology classes were complete and correct as determined 
by human classification. The initial first-pass automated classification phase was useful because it reduced the labor involved in human classification since many images were classified correctly. Removing mistakes from a classification category is a simpler task than choosing a category for each image from scratch and is therefore less time-consuming and likely less prone to error.

Some images remained unsorted by the software, and these were manually classified. Additionally, there were cases in which multiple overlapping images were saved from a given frame. These were identified by their time-stamps and inspected to ensure that the same colony was not counted as Trichodesmium multiple times.

Data from the spring cruise were processed following a similar procedure. However, the image collection rate in the spring was much higher due to greater abundance of observable objects in the water as well as adjustments to the VPR field of view resulting in a larger sample volume. The number of images saved per hour reached as high as 17,000. In addition, VPR sampling was more extensive during the spring cruise. The greater number of images saved meant that human intervention for the entire data set would be prohibitively time-consuming.

Due to the large number of images and the necessity of time-consuming human intervention in the classification procedure, only a subset of the sampled images were classified. First, the relationship between sampling rate and error was investigated. All images from a single sampling hour were classified. Subsets of varying size were constructed, and the percent deviation of the resulting mean abundance from that of the full dataset was assessed for each Trichodesmium colony class as well as for copepods (Figure 2-4). A sampling rate of 1000 images per hour was chosen as a compromise between processing time and accuracy; at a subset size of 1000 images the deviation of abundances of puffs, rafts, and bowties dropped below 20\%; errors in copepod abundances from the subsampled data set were larger, but this taxa will not be analyzed.

One thousand images were selected from each hour by ordering the images chronologically and choosing every $n^{\text {th }}$ image, where $n$ is equal to the total number of images 


\section{Percent Deviation in Mean Abundance Estimate from Full Sample vs. Subsample Size, by Taxa}

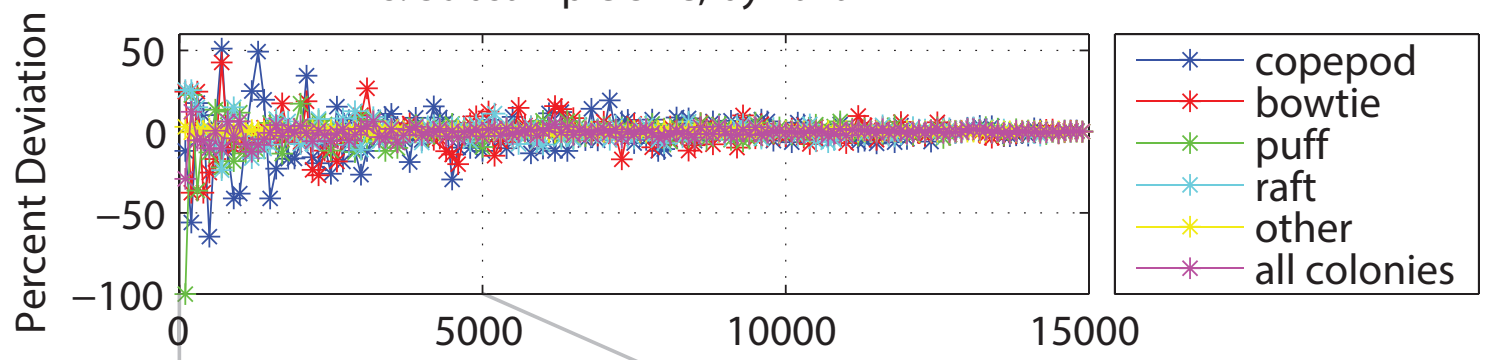

Number of Images Included in Abundance Estimate

Percent Deviation in Mean Abundance Estimate from Full Sample

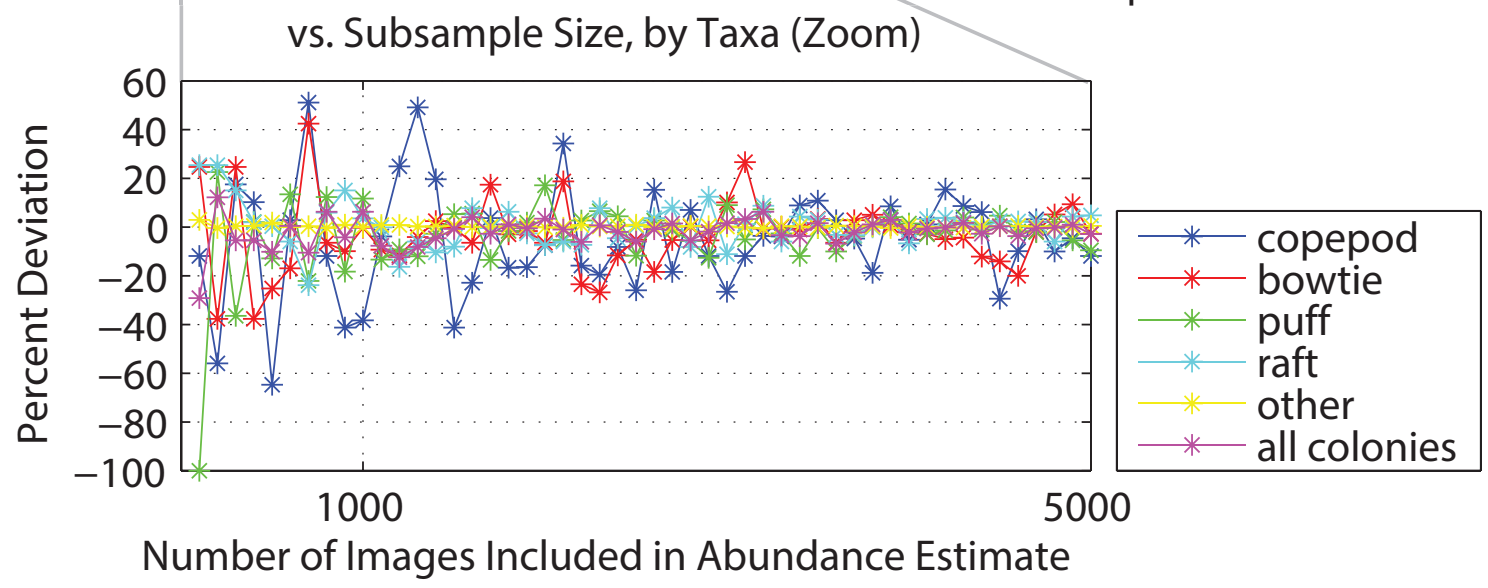

Figure 2-4: Percent deviation of mean abundance from full population estimates in data from a single hour of sampling as a function of subsample size. All 14,892 images collected in one hour by the VPR were sorted into Trichodesmium morphology, copepod or other categories. $\mathrm{N}$ evenly spaced images were then sampled, where $\mathrm{N}$ ranged from 100 to 14892, in increments of 100, and the resulting abundance estimates calculated.

in the hour divided by 1000 . This means that within each hour, areas with a higher density of images were sampled with higher frequency. This improved resolution of the high-abundance areas that are often of greater interest, but at the same time introduced a bias toward reduced sampling of relatively unpopulated regions in the same hour as other highly populated regions. This must be kept in mind when interpreting the resulting data set. For instance, in one region with a low-salinity freshwater lens, almost all objects identified were from the freshwater lens rather than the water below. Thus, the region below the lens is sampled much more sparsely than a 
similarly populated region in another hour with consistently low image abundance. The probability of observing rare species is reduced as the sampling rate is reduced, and therefore zero abundance sampled in this region is not necessarily equivalent to zero abundance sampled elsewhere. The effective limit of detection varies with sampling frequency. Similarly, random noise is expected to increase at lower sampling frequency. Caution must therefore be exercised in interpreting the resulting spring abundance data. However, variation in sampling frequency was preferable to global under-sampling of low-abundance regions, and sampling at a higher constant rate was impractical.

Because multiple images were selected per VPR vertical towing cycle, and because the selection frequency varies due to its dependence on the variable number of images per frame, spatial aliasing is unlikely. Furthermore, periodic sampling of a random distribution will still result in a random distribution, and it is assumed that the distributions of Trichodesmium colonies and other objects are random over the spatial and temporal scales over which subsampling occurs.

The SVM classifier, trained with spring 2011 data, was used for the initial processing of the subset of spring 2011 images. Then, classifications were manually corrected, unclassified images belonging to the subset added, and duplicate images removed, as was done for the full set of images from fall 2010.

Following classification, abundances were estimated from the frequency of observation of each taxa. Typically, this is done over 1-second intervals using the standard VPR software. Within each 1-s interval, the number of observations is divided by the sample volume per second. This sample volume is normally estimated by multiplying the frame rate (frames $\mathrm{s}^{-1}$ ) by the image volume $\left(\mathrm{L}\right.$ frame $\left.\mathrm{e}^{-1}\right)$. The volume of the field of view of was calibrated before each cruise by the tethered copepod method described by Davis et al. (2005) and was $6.929 \mathrm{~mL}$ and $15.168 \mathrm{~mL}$ for OC469 and OC471, respectively. The corresponding vertical and horizontal dimensions of the field of view were $13 \mathrm{~mm}$ x $13 \mathrm{~mm}$ and $12 \mathrm{~mm}$ x $12 \mathrm{~mm}$; the camera and strobe were oriented to produce a deeper and narrower field of view for OC471. 
In order to estimate abundances based on the classified subsample from spring 2011, we adjusted the sample volume by multiplying by the fraction of images classified within each period. However, one-second bins were too small to appropriately resolve abundances based on the subsample of data. Too few classified images were present in each bin to apply the volume correction at that scale. After subsampling, most of the one-second intervals had only 0,1 , or 2 images in them.

To resolve this problem, the data were binned to a coarser grid. Binning the data to a coarser grid has the additional advantage of aiding in visualization of patterns in Trichodesmium colony abundance (Figure 2-5). Therefore, the method was applied to the fully classified fall 2010 data as well.

To construct coarse-resolution abundance fields for the fall 2010 data, bins were constructed to include data from multiple vertical cycles of the VPR (Figure 2-5). A bin size of $5 \mathrm{~m}$ in the vertical by $11 \mathrm{~km}$ in the horizontal was chosen for the fall data to include approximately three vertical cycles of the VPR per horizontal bin length. Larger bins of $5 \mathrm{~m}$ in the vertical by $33 \mathrm{~km}$ in the horizontal were chosen for the spring data to compensate for the reduced observation frequency of the subsampled data.

The binning procedure for the fully-classified fall data was as follows. First, the images were binned in one-second intervals so that they could be associated with a latitude, longitude, and depth from the one-second VPR CTD data. An along-track distance was calculated as a running sum of the distance in kilometers between each consecutive latitude-longitude pair. Spatial bins were defined by intervals in vertical space and along-track distance, so that many temporal bins $i$ fell in each spatial bin $m$ (as in the right panel of Figure 2-5).

Within each bin, abundances were estimated under the assumption of a Poisson distribution, based on the total water volume imaged and the number of positive 


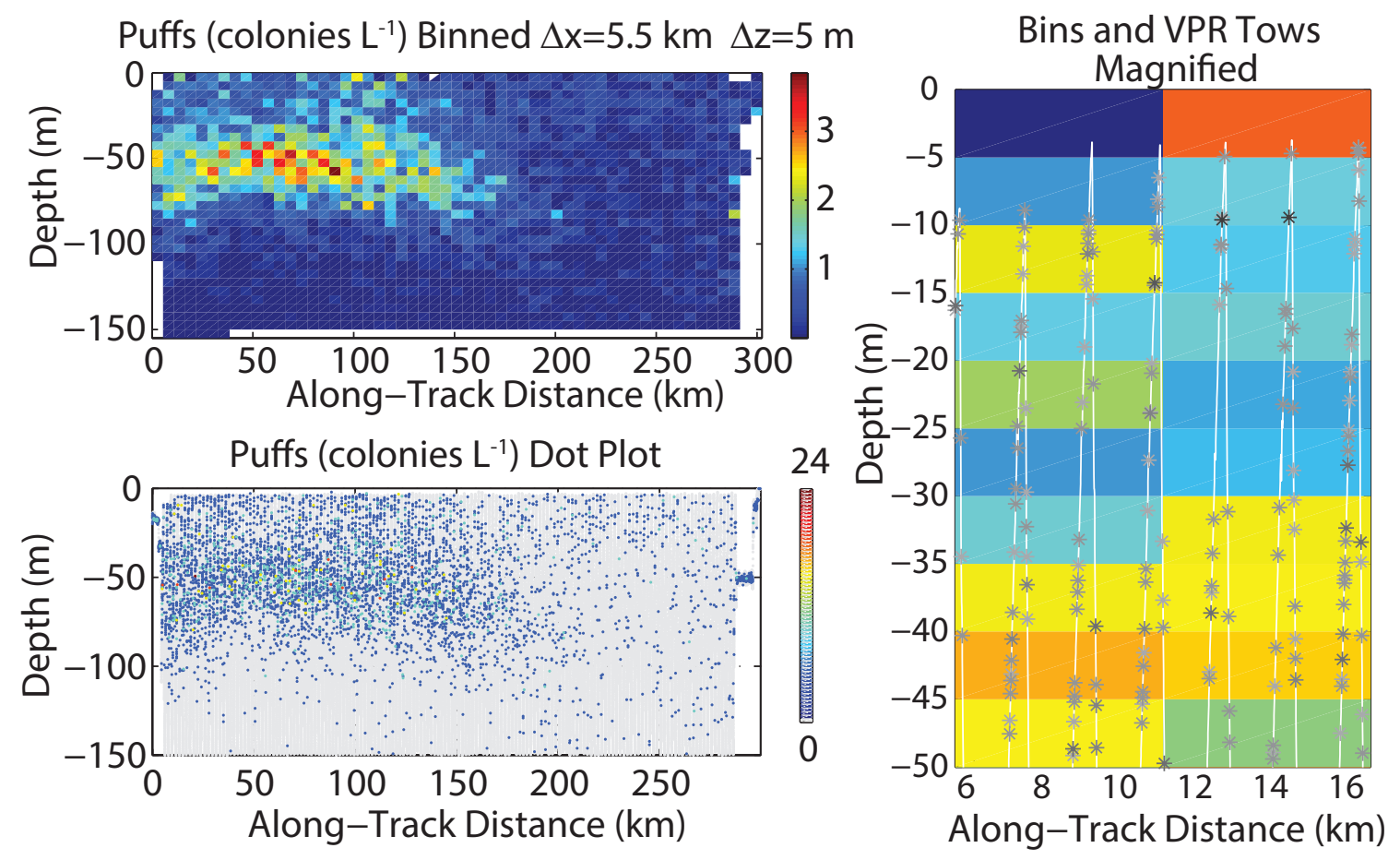

Figure 2-5: Illustration of binning method. Bottom left panel: VPR data in 1-s bins. Because the bin size is so small, each bin contains zero, one or two colonies. Upper left panel: coarsely binned VPR data, gridded to $5.5 \mathrm{~km} \times 5 \mathrm{~m}$ bins, facilitating perception of gradients by eye. Right panel: magnified view of VPR tracks with 1-s binned data overlaid on coarsely binned data. 
identifications. For each spatial bin $m$, an index $f_{m i}$ was defined so that

$$
f_{m i}= \begin{cases}1 & \text { if } 1 \text {-s interval } i \text { falls in the spatial bin } m \\ 0 & \text { otherwise }\end{cases}
$$

Then, the abundance in each spatial bin $m$ was calculated by the formula

$$
C_{m}=\frac{\sum_{i=1}^{N} f_{m i} n_{i}}{\sum_{i=1}^{N} f_{m i} V_{i}}
$$

where $N$ is the total number of temporal bins $i, n_{i}$ is the number of images of the category of interest in bin $i$. For the fall 2010 data, $V_{i}$ was a constant equal to $V$, the volume sampled in each interval $i$, determined as before from the image volume and number of frames per second. The variance of the abundance in each bin is equal to the abundance estimate divided by the sample volume:

$$
\operatorname{var}\left(C_{m}\right)=\frac{\sum_{i=1}^{N} f_{m i} n_{i}}{\left(\sum_{i=1}^{N} f_{m i} V_{i}\right)^{2}}
$$

The binning is carried out the same way for the subsampled data from spring 2011 except that for each bin $i$ an individual volume estimate is made based on the fraction of the total number of images in the interval that were subsampled. Thus, for spring 2011,

$$
V_{i}=\frac{s_{i}}{a_{i}} V
$$

where $V$ is defined as before but $s_{i}$ is the total number of subsampled images in interval $i$ and $a_{i}$ is the total number of images in interval $i$ prior to subsampling.

For a 165-km long segment of the spring 2011 dataset, all images were classified. Abundances resulting from the fully classified data were compared to those based on 
the 1000 image per hour subset at varying grid resolutions.

\subsubsection{Comparison of Abundances from VPR and Microscopic Enumeration}

Microscopic colony counts were compared to VPR colony abundances to confirm the calibration of the VPR sampling volume on both cruises and the image subsam-

pling method applied to the Spring 2011 cruise as well as the overall consistency of VPR with traditional methods. The VPR was towed between stations but had to be deployed and recovered while the ship was underway, before and after stations. Therefore, VPR sampling did not directly overlap in space and time with sampling of water at stations for microscopic enumeration of colonies. In order to carry out a comparison of the two methods, we have paired each water sample with the binned average of nearby VPR data. The horizontal extent of each bin was chosen to encompass the nearest $33 \mathrm{~km}$ (Spring 2011) or $22 \mathrm{~km}$ (Fall 2010) of VPR track to each station location. Vertically, the bins extended $5 \mathrm{~m}$ above and below each bottle sample depth. All bottle samples for which $33 \mathrm{~km}$ of VPR data were not available within a $30 \mathrm{~km}$ radius were excluded. Due to the spatial and temporal separation of available VPR data from the locations and times of water sample collection, we do not expect exact correspondence between the two data types, even if both measured colony abundance with 100\% accuracy. The comparison instead provides an opportunity to look for large systematic variation between the two methods.

\subsection{Results and Discussion}

\subsubsection{Comparison of Subsampled with Fully Classified Data}

Abundance estimates based on the subsampled and full datasets were compared over a 165-km segment of the spring 2011 VPR track. Reduced sampling rate through subsampling leads to increased noise in the data. However, this tendency is counter- 
acted to some extent by increasing the bin size over which abundance is estimated so that a greater number of samples are included per bin. The random error introduced in the estimate decreases with increased bin size (Figure 2-6).

Despite additional noise at lower sampling frequency, broad spatial patterns on the scale of several bins are consistent between fully-classified and subsampled data sets (Figure 2-7). A linear relationship between abundance in each grid cell based on the subsampled and fully classified datasets exhibits a nearly 1:1 slope but with significant added noise in the estimates based on the subsample (Figure 2-8, left panel). This random noise tends to cancel in operations involving addition of abundance estimates from multiple grid cells. For instance, there is much less noise in the relationship between depth-integrated abundance from the two methods (Figure 2-8, right panel). Additionally, average vertical distributions derived from the subsampled data are consistent with, and only slightly noisier than, the corresponding patterns calculated from the fully-classified data (Figure 2-9). Thus, the subsampled data is sufficient for assessing the mean vertical colony distributions that are of primary interest in this study.

The relative error between subsampled and fully-classified data for the three Trichodesmium colony morphologies may be as high as 136\% for a given bin (Table 2.2). Thus, subsampled data should be interpreted with care. Broad patterns can be identified, but specific values at individual points are less reliable.

\subsubsection{Comparison of Abundance Estimates: VPR vs. Micro- scopic Enumeration}

We compare $11 \mathrm{~km}$ (Fall 2010) or $33 \mathrm{~km}$ (Spring 2011) × $20 \mathrm{~m}$ binned VPR data with microscopic counts for nearest data pairs. If a full bin is not available within $30 \mathrm{~km}$ of a microscopic count location, that station is excluded from analysis. A two-sample Kolmogorov-Smirnov test does not at the 5\% significance level reject the hypothesis that the VPR and microscopically enumerated abundance data come 


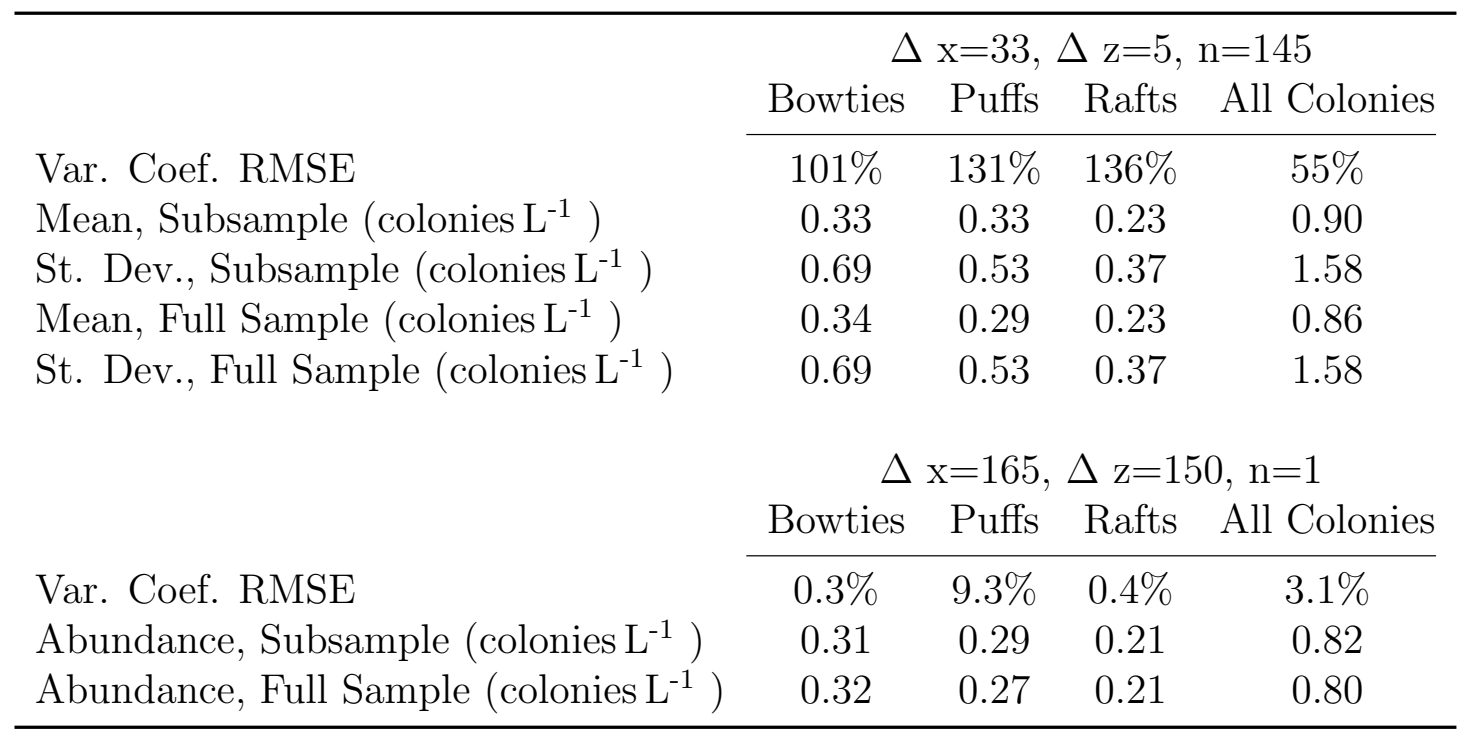

Table 2.2: Comparison of binned abundance estimates based on subsample and fully classified data sets. Statistics are calculated for $33 \mathrm{~km} \times 5 \mathrm{~m}$ bins and for a single bin encompassing the entire $165 \mathrm{~km}$ region for which full classification was completed (33 $\mathrm{km} \times 5 \mathrm{~m}$ binned data displayed in Fig. 2-7). Variation coefficient of the root mean square error is calculated as $\sqrt{\frac{\sum^{n}\left(A_{\text {sub }}-A_{\text {full }}\right)^{2}}{n}} / \operatorname{mean}\left(A_{\text {full }}\right)$, where $A$ denotes abundance. Also shown are the mean and standard deviation of the binned abundance, or the actual abundance in the case of the single large bin. 


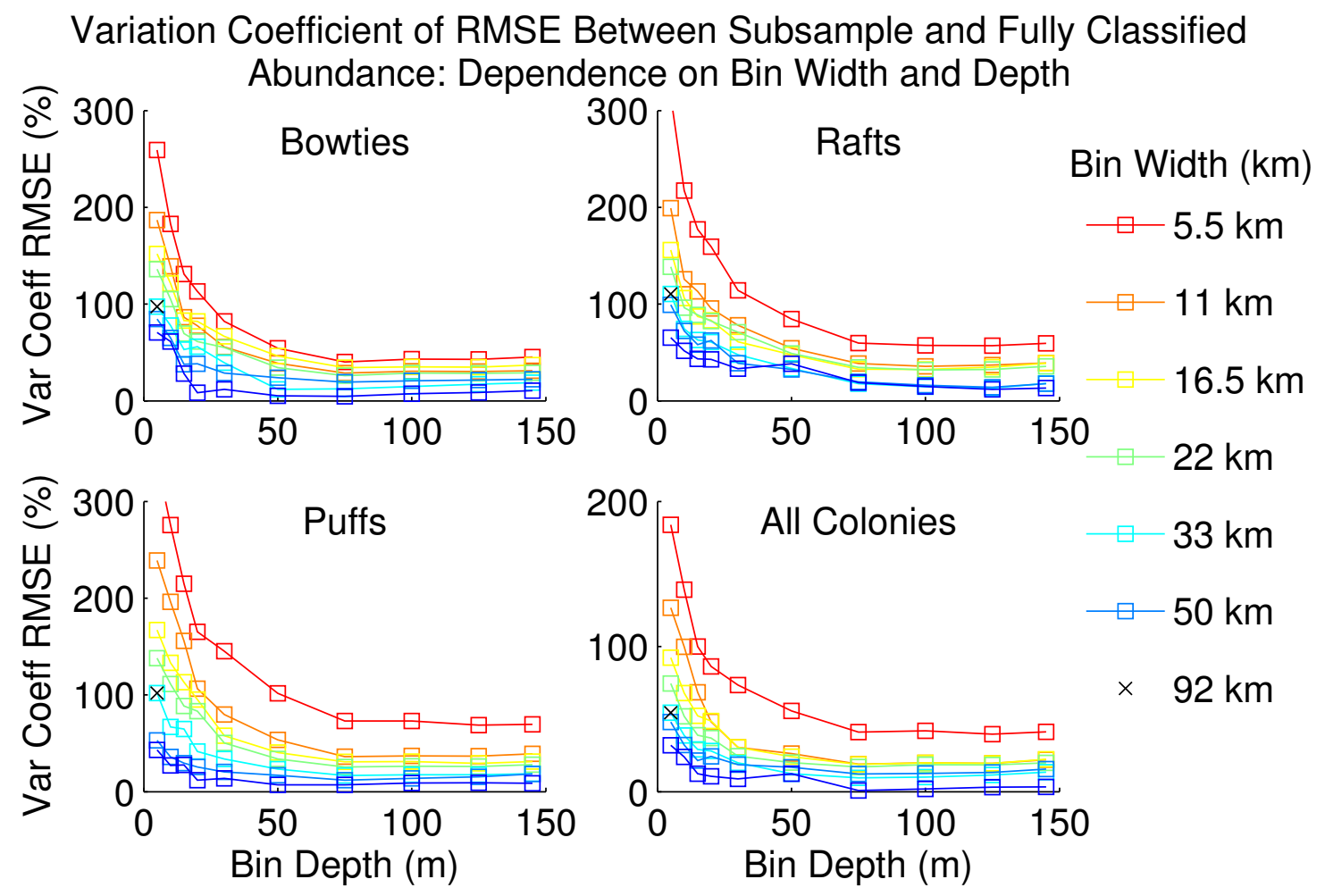

Figure 2-6: Variation coefficient of root mean square error of abundance based on subsample compared to fully classified data $\left(\sqrt{\left(A_{s}-A_{0}\right)^{2} / N} / \operatorname{mean}\left(A_{0}\right)\right)$ for various horizontal and vertical bin dimensions. Selected bin size of $5 \mathrm{~m} \times 33 \mathrm{~km}$ is marked with an ' $x$ ' in each panel. 


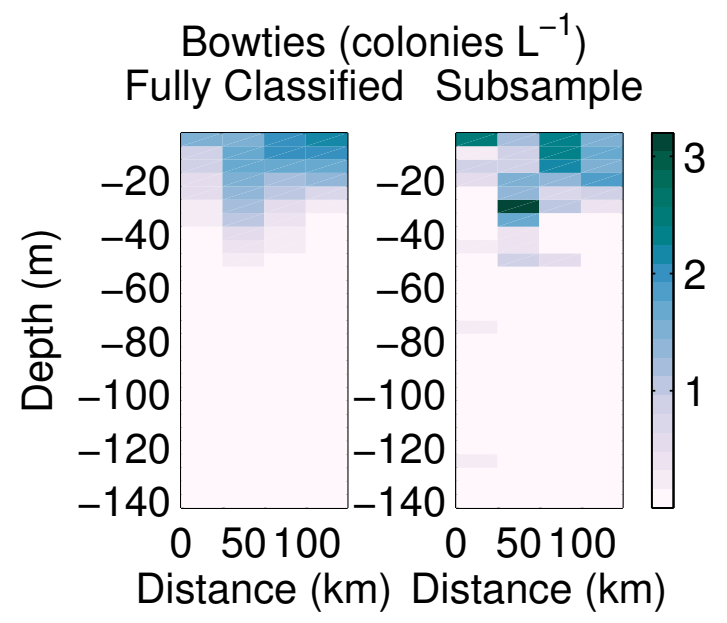

Puffs (colonies $\mathrm{L}^{-1}$ )

Fully Classified Subsample

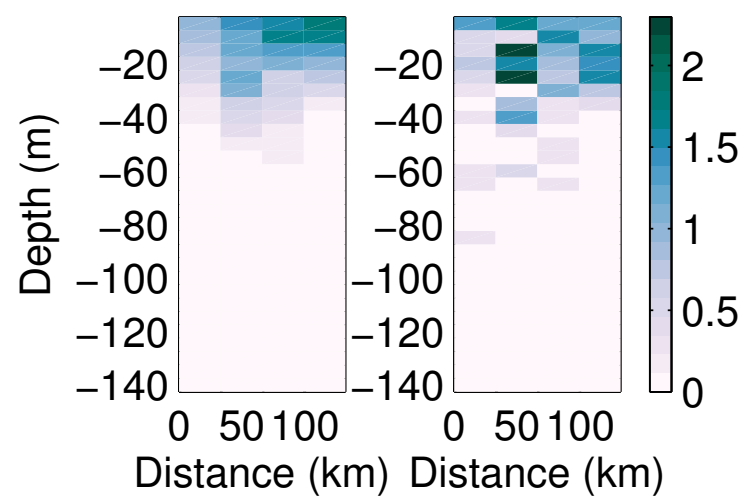

Rafts (colonies $\mathrm{L}^{-1}$ ) Fully Classified Subsample

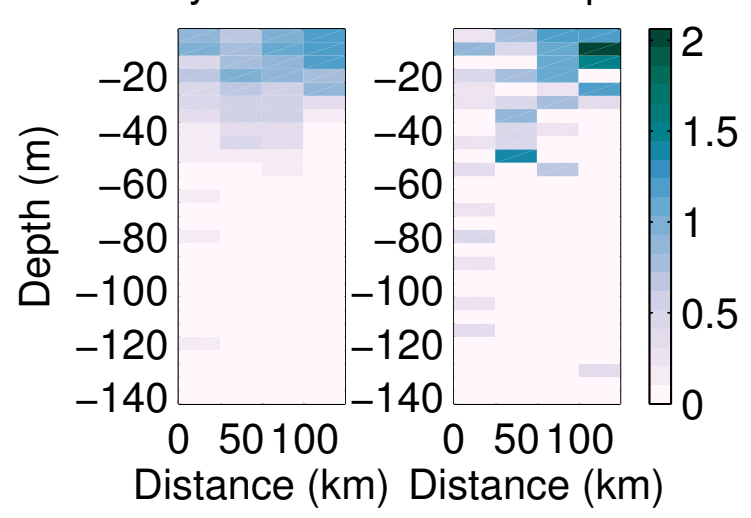

All Colonies (colonies $\mathrm{L}^{-1}$ ) Fully Classified Subsample

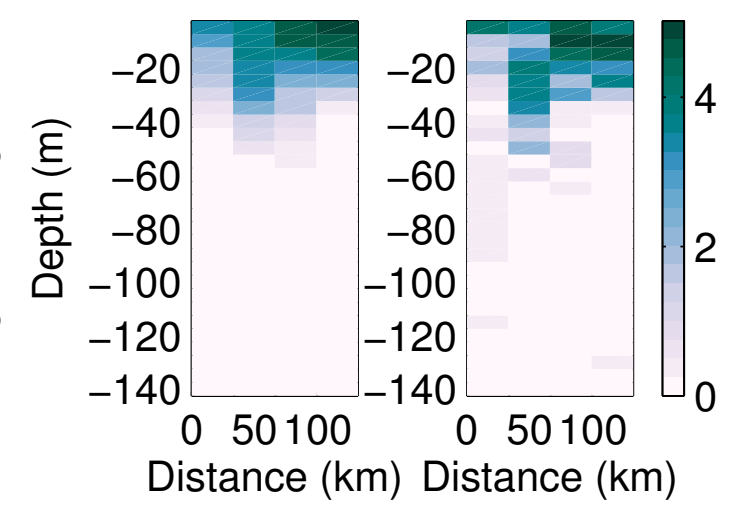

Figure 2-7: Comparison between fully-classified and subsampled binned Trichodesmium colony populations from OC471 by morphology. 

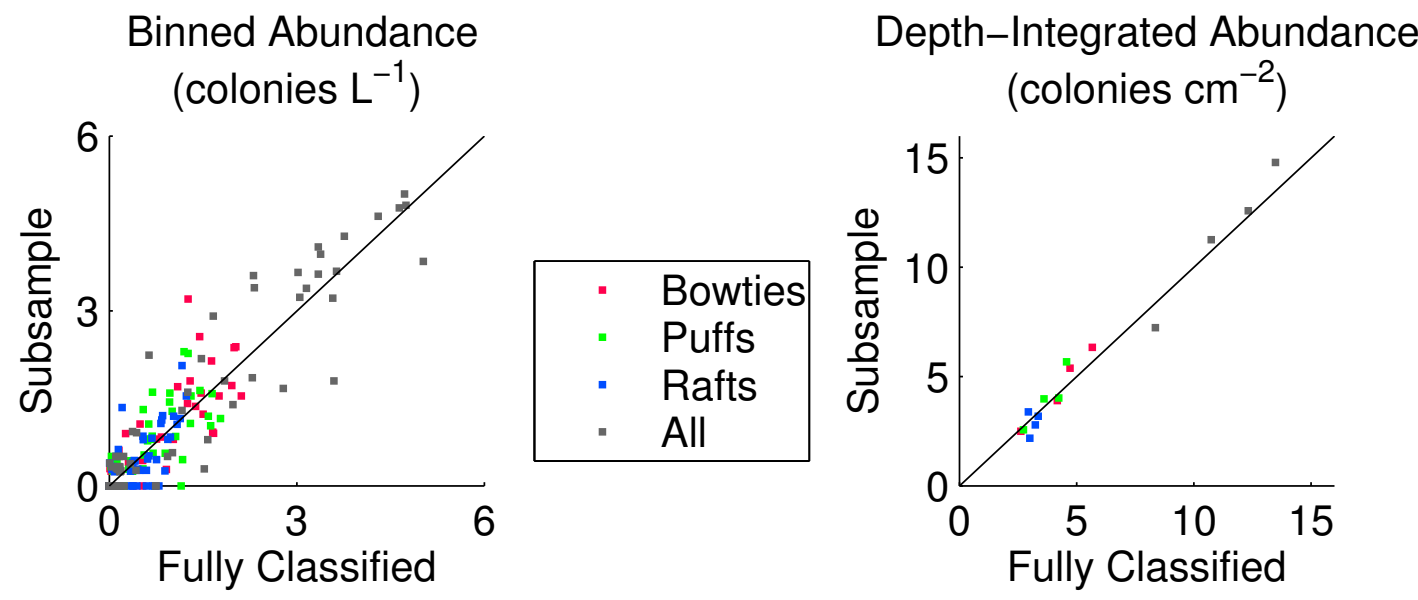

Figure 2-8: Comparison between fully-classified and subsampled binned Trichodesmium colony abundances from OC471 by morphology. Left panel shows abundance estimates from each $5 \mathrm{~m} \times 33 \mathrm{~km}$ bin. Right panel shows integrated abundance estimates calculated from the $5 \mathrm{~m} \times 33 \mathrm{~km}$ binned data. 

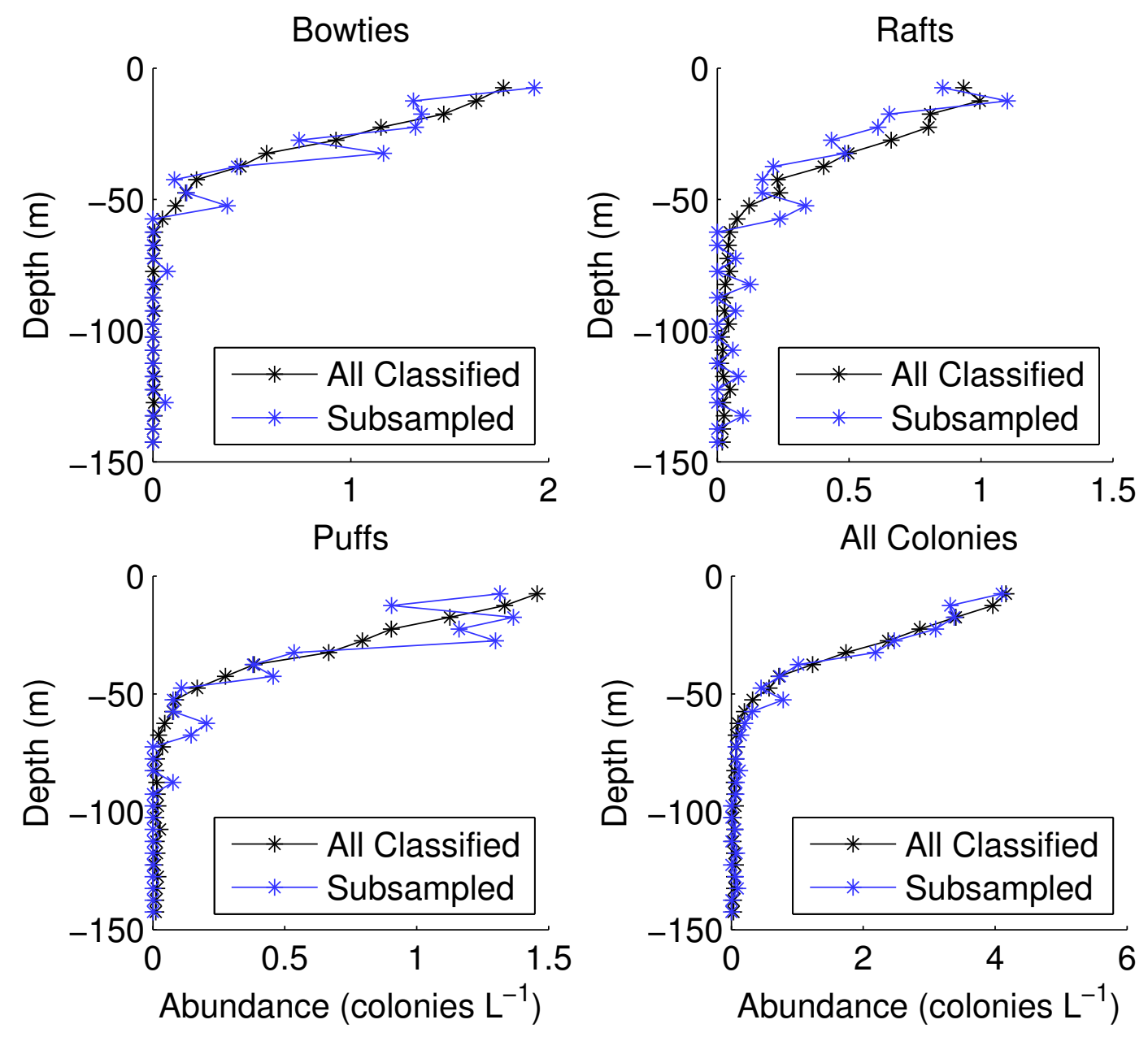

Figure 2-9: Comparison of depth profiles based on fully-classified and subsampled binned raft populations for the data section displayed in Fig. 2-7. 
from the same continuous distribution for either cruise or for the cruises combined. Histograms displaying the distribution of abundance estimates reveal only small differences in abundance distribution between the two methods (Figure 2-10). However, in the Spring 2011 data, which reaches higher abundance levels than the Fall 2010 data, the data obtained by microscopic enumeration have more instances at higher abundance than were found using the VPR. This pattern is even more evident when VPR-based abundance is plotted versus abundance from microscopic enumeration (Figure 2-11). Agreement between the two methods shows variability on either side of the 1:1 line for small abundance (below about 2 colonies $\mathrm{L}^{-1}$ ). At higher abundances, as assessed by microscopic enumeration, nearby VPR abundance estimates are consistently lower.

One possible explanation for these low VPR estimates in regions of high abundance is lost images. When the number of images per frame becomes too large, the system is unable to keep up with the rate of image creation, and some images are not saved. This occurred more frequently in Spring 2011 cruise than Fall 2010 cruise. The total number of images detected per frame is unknown since there is no record of the lost images. Instead, we compare the bias in VPR-based abundance (relative to microscopic enumeration) with the number of images saved per second in the Spring 2011 data. As the number of images recorded per second increased past about 25 images $\mathrm{s}^{-1}$, the bias in the VPR abundance estimate increased dramatically (Figure 2-12). However, this does not necessarily indicate a causal link between from frame rate to bias; bias increases at higher Trichodesmium abundance, and higher Trichodesmium abundance often coincides with a higher total number of images.

Another factor that may contribute to the reduced frequency of observation of the highest abundance levels in the VPR dataset is the fact that each VPR estimate corresponds to an average of samples taken at several distinct locations, whereas the microscopic enumeration samples come from a single location. Given the patchy nature of Trichodesmium colony distributions at small scales, the probability of capturing a single high abundance patch in a water sample from one location is higher 
than the probability of capturing multiple abundance patches within a binned region of the VPR sample.

Histograms of Abundance Measured by VPR and Microscopic Enumeration Fall 2010
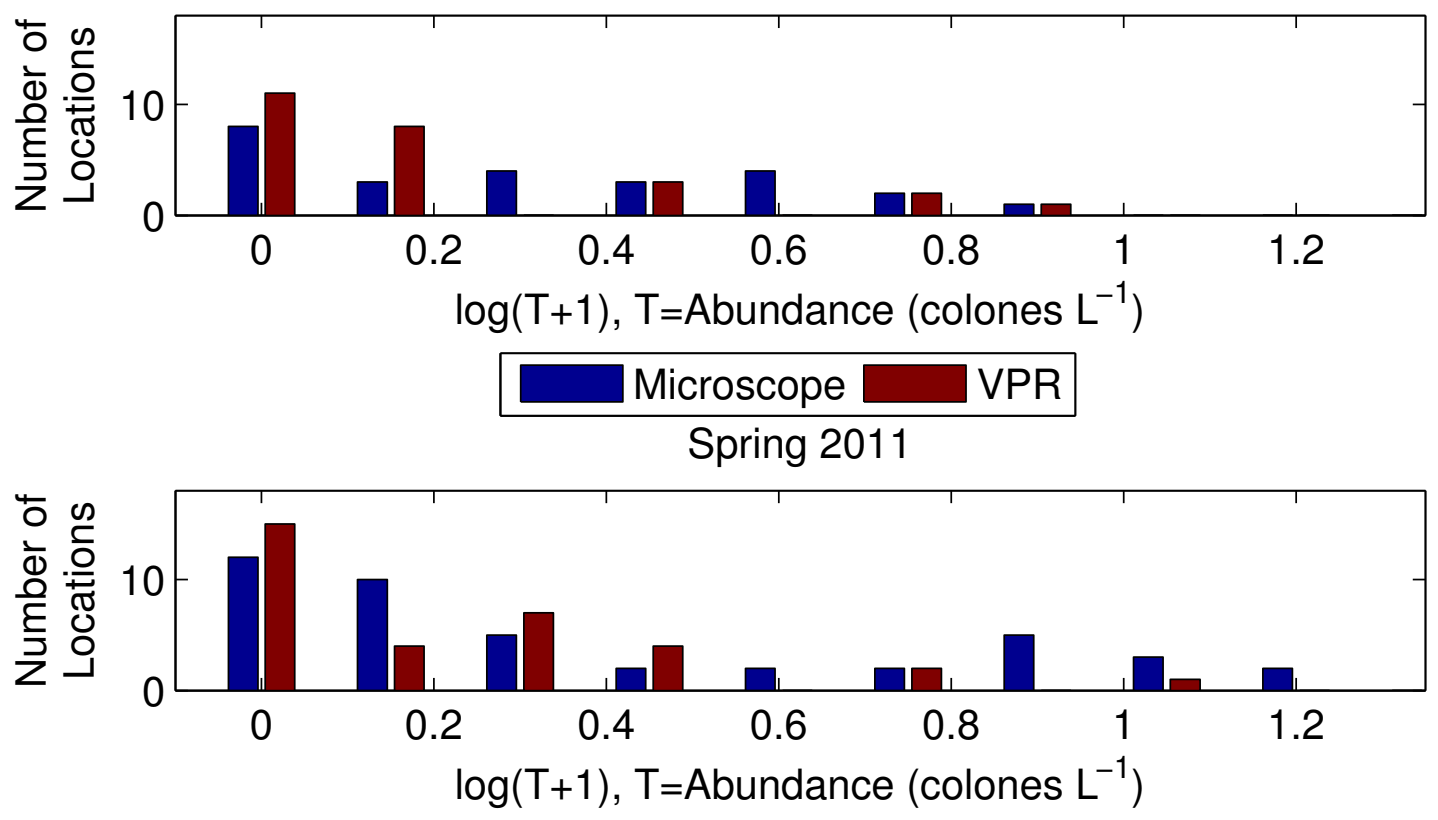

Figure 2-10: Histograms showing the distribution of abundance measurements as assessed by the VPR and by microscopic enumeration at paired locations. Samples used for microscopic enumeration at stations are paired with binned means of nearby VPR data.

\subsection{Conclusions}

A reduced-labor method was developed for the extraction of abundance of rare taxa from high volume VPR datasets. Comparisons between regional abundance estimates based on the full set of images and the subset demonstrate consistency in the mean but added noise in the subsample-derived fields at the bin level. The additional noise due to subsampling is random, whereas error due to false positive identifications by automated classification error can be systematic, reflecting patterns in abundance of other taxa. At a bin size of $5 \mathrm{~m}$ by $33 \mathrm{~km}$, the noise is comparable to 


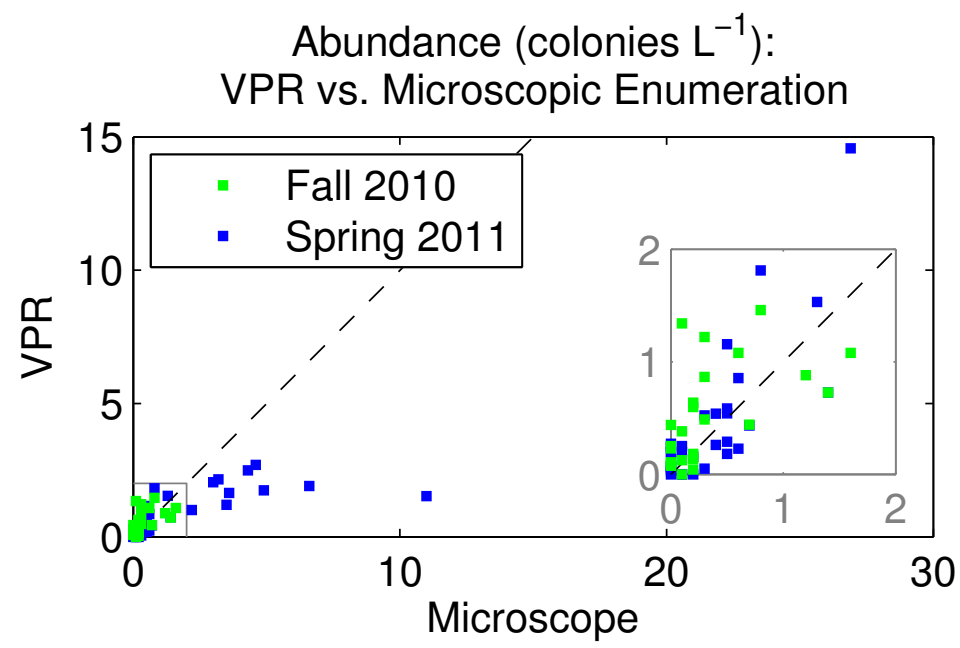

Figure 2-11: Comparison between estimates of Trichodesmium colony abundance based on the VPR and microscopic enumeration for nearby measurements. Samples used for microscopic enumeration at stations are paired with binned means of nearby VPR data. Inset shows enlargement of the lower right corner of the plot, showing abundances less than 2 colonies $\mathrm{L}^{-1}$.

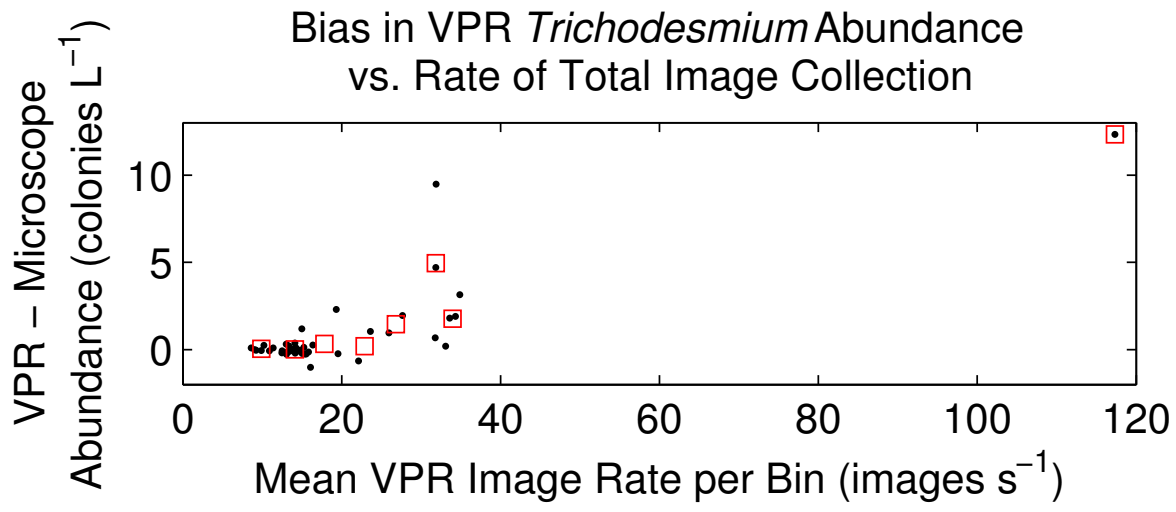

Figure 2-12: Bias in VPR-estimated abundance assessed by comparison with abundances from microscopic enumeration, plotted versus VPR rate of image acquisition. Red squares represent binned mean values at 10 image $\mathrm{s}^{-1}$ intervals. 
the magnitude of the abundance for each of the three colony morphologies. However, the same broad patterns in abundance are identifiable in both the fully classified and subsampled estimate. Additionally, in operations involving summing abundances over multiple grid cells, the noise tends to cancel so that the resulting quantities are roughly consistent with those calculated from the fully classified set. The noise introduced by the subsampling method does not introduce significant bias as evidenced by the consistency in mean and integrated abundances between the two methods.

Based on comparison with the traditional method of microscopic enumeration, VPR estimates of Trichodesmium colony abundance are consistent at low abundance $\left(<2\right.$ colonies $\left.\mathrm{L}^{-1}\right)$. At higher abundance levels, which are only present in the subsampled spring 2011 data, the VPR estimates are skewed low but still tend to increase with increasing abundance. At high abundance, VPR-based estimates are likely underestimates of the true abundance. However, the VPR method is able to capture large signals in abundance even in higher abundance regions. Although the sensitivity to changing abundance appears to be somewhat reduced and noise somewhat increased at high abundance levels, there is still a broad tendency for VPR estimates to increase with increasing microscopic abundance. Part of the difference between the two methods may be attributed to the broader sampling area included in each VPR estimate, which makes the method less likely to capture small local highs in abundance associated with small-scale patchiness in Trichodesmium colony distributions. Comparisons at consistent spatial scales could resolve the question of possible inconsistency at high abundance but cannot be carried out with available data. 


\section{Chapter 3}

\section{Mesoscale Eddies and}

\section{Trichodesmium Abundance}

\subsection{Abstract}

Correlations of Trichodesmium colony abundance with the eddy field emerged in two segments of Video Plankton Recorder observations made in the southwestern North Atlantic during fall 2010 and spring 2011. In fall of 2010, local maxima in abundance were observed in cyclones. We hypothesized Ekman transport convergence as a mechanism for trapping buoyant colonies in cyclones. Conversely, Ekman transport divergence could tend to dilute colony concentrations in anticyclones. Idealized models supported the potential of this process to influence the distribution of buoyant colonies. However, while the process may have been a factor in the development of patterns observed in fall 2010, idealized simulations did not reproduce the full magnitude of the observed pattern. Elevated abundances in anticyclones in the spring were correlated with anomalously fresh water, suggesting riverine input as a driver of the relationship. 


\subsection{Introduction}

Trichodesmium is a genus of nitrogen-fixing cyanobacteria found throughout the tropical and subtropical oceans. Converting atmospheric dinitrogen into biologically available forms, it represents an important source of new nitrogen to oligotrophic regions and contributes to new production. Since nitrogen often limits primary production, nitrogen made available through fixation by Trichodesmium contributes to primary production and potentially to carbon export from the surface waters. Thus, untangling the controls on Trichodesmium abundance and distribution is a step toward quantifying the contribution of Trichodesmium to oceanic nitrogen and carbon cycling.

On a video plankton recorder (VPR) survey across the North Atlantic subtropical gyre, Davis and McGillicuddy (2006) identified a correlation between Trichodesmium colony abundance and sea level anomaly. Greater abundances were observed in anticyclones. Other observations of elevated Trichodesmium abundance in anticyclones have been made in the Pacific (Fong et al., 2008) and eastern North Atlantic (Taboada et al., 2010). To investigate the spatial and temporal generality of this pattern, we analyzed relationships between sea level anomaly and abundance based on VPR data from two cruises, in Fall 2010 and Spring 2011 in the high abundance southwestern North Atlantic. There was no single ubiquitous relationship between mesoscale dynamics and colony abundance. However, patterns emerged within localized regions on the two cruises, with elevated abundances in anticyclonic eddies on the spring cruise and cyclonic eddies on the fall cruise. Here we attempt to explain these patterns through analysis of the physical processes underlying each. We suggest that the association with anticyclones may be driven by nutrient supply through riverine freshwater input. We develop a hypothesis of wind-driven physical aggregation cyclones and dilution in anticyclones to explain the association of colony abundance with cyclones, and we evaluate the effects of the proposed dynamic using idealized eddy models. These two mechanisms of creating mesoscale variability in Trichodesmium 
populations are distinct from that described in Davis and McGillicuddy (2006).

We completed several numerical experiments in order to test the hypothesis that buoyant particles, representing Trichodesmium colonies, could be concentrated in cyclones and diluted in anticyclones by convergence and divergence, respectively, of Ekman transport due to wind stress curl associated with eddy surface currents (Figure 3-1). Well-developed theory predicts surface convergence in cyclones (Dewar and Flierl, 1987; Martin and Richards, 2001). Wind-driven surface currents converge at the center of cyclones due to variations in wind stress across the breadth of an eddy. These wind stress variations result from the difference in the surface currents on opposite sides of the eddy. Since wind stress depends on the relative velocity between air and ocean, the stress will be greater on the side where the currents flow opposite to the wind. As a result, in the boundary layer of a cyclone, more water will flow into the cyclone on the side where currents oppose the wind than will flow out on the side where currents flow with the wind. This results in surface convergence, which is balanced by downward motions in the eddy interior. Conversely in anticyclones, divergence produces upwelling in the eddy interior. Vertical velocities due to this type of spatial variation in Ekman transport have been identified previously (McGillicuddy Jr. et al., 2007; Ledwell et al., 2008) as a mechanism for nutrient supply in anticyclonic and mode-water eddies, and the physical mechanism has been well described (Dewar and Flierl, 1987; Martin and Richards, 2001).

Buoyant particles, such as Trichodesmium colonies, will tend to aggregate in regions of surface convergence; they are advected laterally but float rather than following water trajectories leaving the surface (Figure 3-1). Similarly, buoyant particles will become spread out in regions of surface divergence. This process has previously been investigated in the context of frontal dynamics (Franks, 1992). Additionally, submesoscale modeling of effects of surface convergence and divergence on buoyant particle fields has demonstrated expulsion of particles from anticyclones and aggregation in regions of convergence (Zhong et al., 2012). Here, we expect the convergence/divergence of Ekman transport to lead to concentration/dilution in cyclones/anticyclones (Figure 
$3-1)$. Thus, the effect could lead to higher concentrations of buoyant colonies in cyclones than anticyclones, consistent with the observed distribution of Trichodesmium colonies in relation to the mesoscale eddy field in fall 2010. 

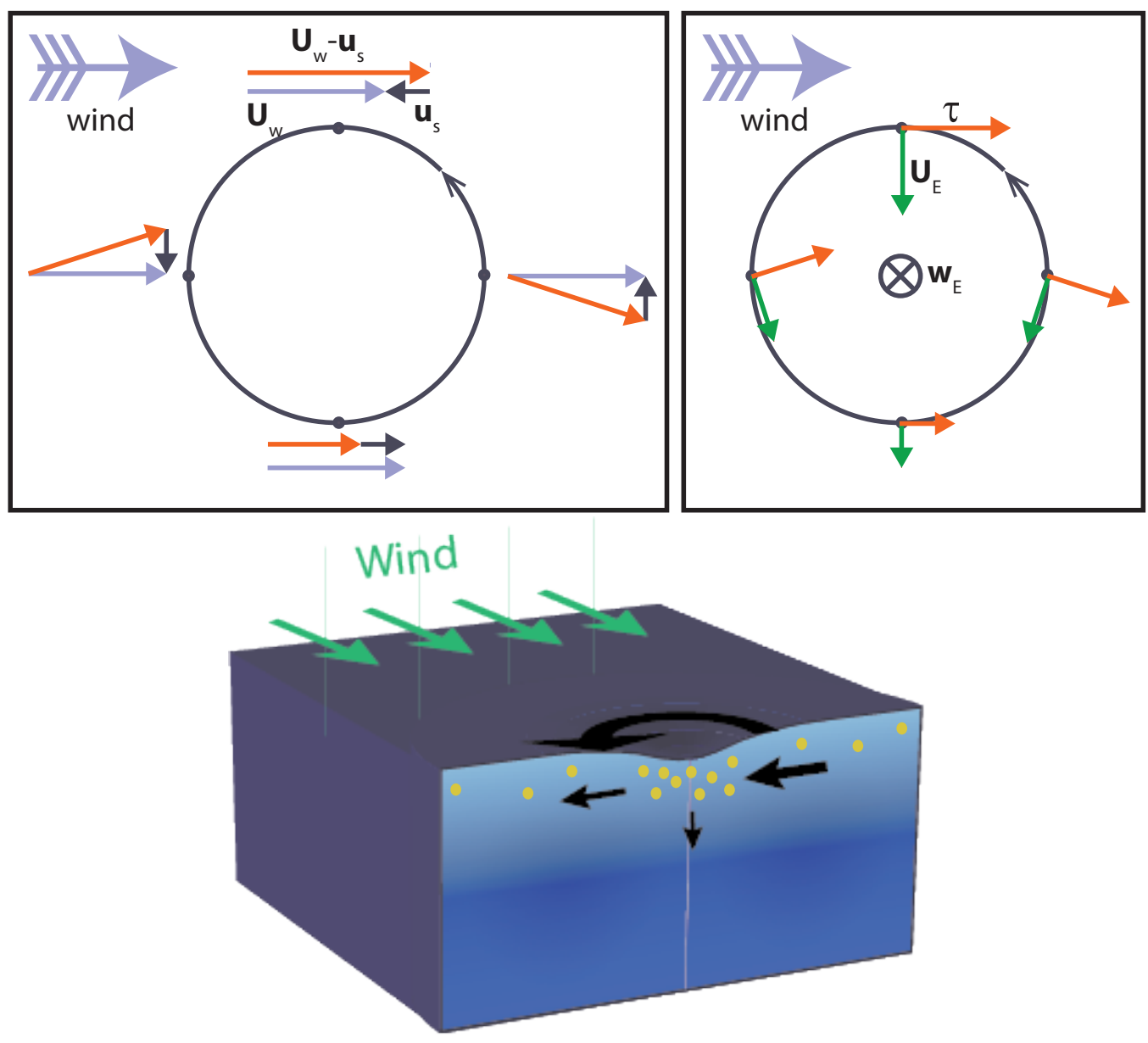

Figure 3-1: Schematic diagram of Ekman convergence concentration of buoyant colonies in a cyclone with wind stress a function of the difference between air and sea surface velocity $\left(\tau \propto\left|U_{w}-u_{s}\right|\left(U_{w}-u_{s}\right)\right)$. The upper left panel shows a plan view of a cyclonic eddy with the difference between wind velocity, $U_{w}$, and sea surface velocity, $u_{s}$, at four points. The upper right panel shows the direction of the resulting wind stress, $\tau$ and Ekman transport, $U_{E}$, at the same locations. Convergence of Ekman transport near the center of the eddy leads to downwelling, $w_{E}$. The bottom panel shows how buoyant colonies could become concentrated in a region of surface convergence and downwelling. 


\subsection{Methods}

\subsubsection{Observational Methods}

Sampling occurred on two cruises on the R/V Oceanus, OC469 from October 122, 2010, and OC471 from April 23-May 13, 2011. The planned cruise track consisted of a meridional section from Bermuda to Puerto Rico, followed by a southeastward leg to a longitude of approximately $40^{\circ} \mathrm{W}$ and a southwestward leg ending just east of Barbados. Precise sampling locations (Figures 3-2 and 3-3) were chosen during the cruises as informed by satellite observations of sea surface height, real-time analysis of VPR Trichodesmium abundance observations, and ocean-color-based estimates of Trichodesmium bloom probability (Westberry and Siegel, 2006; Westberry et al., 2005) provided by Toby Westberry. This adaptive strategy allowed sampling to be directed toward interesting physical and biological features such as eddies and areas of elevated Trichodesmium abundance.

Trichodesmium colony abundance was sampled by the VPR, towed between stations (Figures 3-2 and 3-3). The VPR consists of a towed body, containing a CTD and a synchronized video camera and xenon strobe (Davis et al., 2005). Data are sent in real-time through a fiber-optic cable to a shipboard computer system. The VPR undulates between the surface (5-10 m) and approximately $120 \mathrm{~m}$ depth, completing an up-down cycle approximately every six minutes. Since it is towed at 10 knots (5.1

$\mathrm{m} \mathrm{s}^{-1}$ ), this corresponds to one vertical cycle every $1.8 \mathrm{~km}$. Navigation to starboard of the ship's wake near the surface, in addition to strategic camera and strobe placement and towfish design, minimize disturbance of the regions sampled. At a frame rate of $30 \mathrm{~Hz}$, each 984 x 1009 pixel video frame is passed through object-identification software to pick out "regions of interest", which are saved, using a time-stamp naming convention, to a hard disk.

Images were initially sorted using image recognition software $(\mathrm{Hu}$ and Davis, 2006). For the spring cruise, due to the large number of images collected, the sample size was reduced to 1000 images per sampling hour, evenly spaced among the 


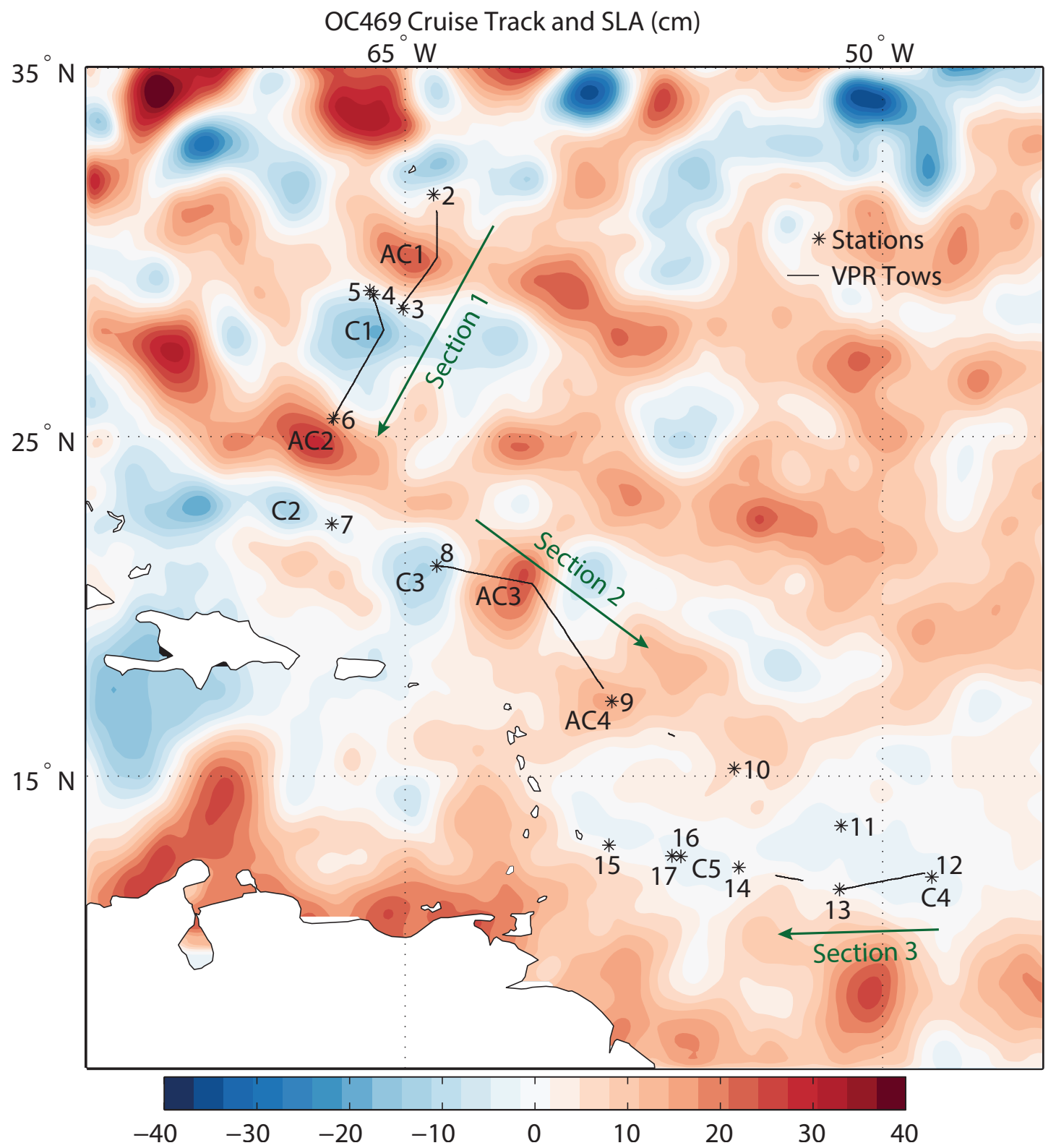

Figure 3-2: Locations of stations and VPR tows for cruise OC469 with sea level anomaly. Black lines indicate VPR tows. Stars indicate station locations. Targeted cyclones and anticyclones are labeled with prefixes "C" and "AC", respectively. Labeled sections are referred to in subsequent analysis. 


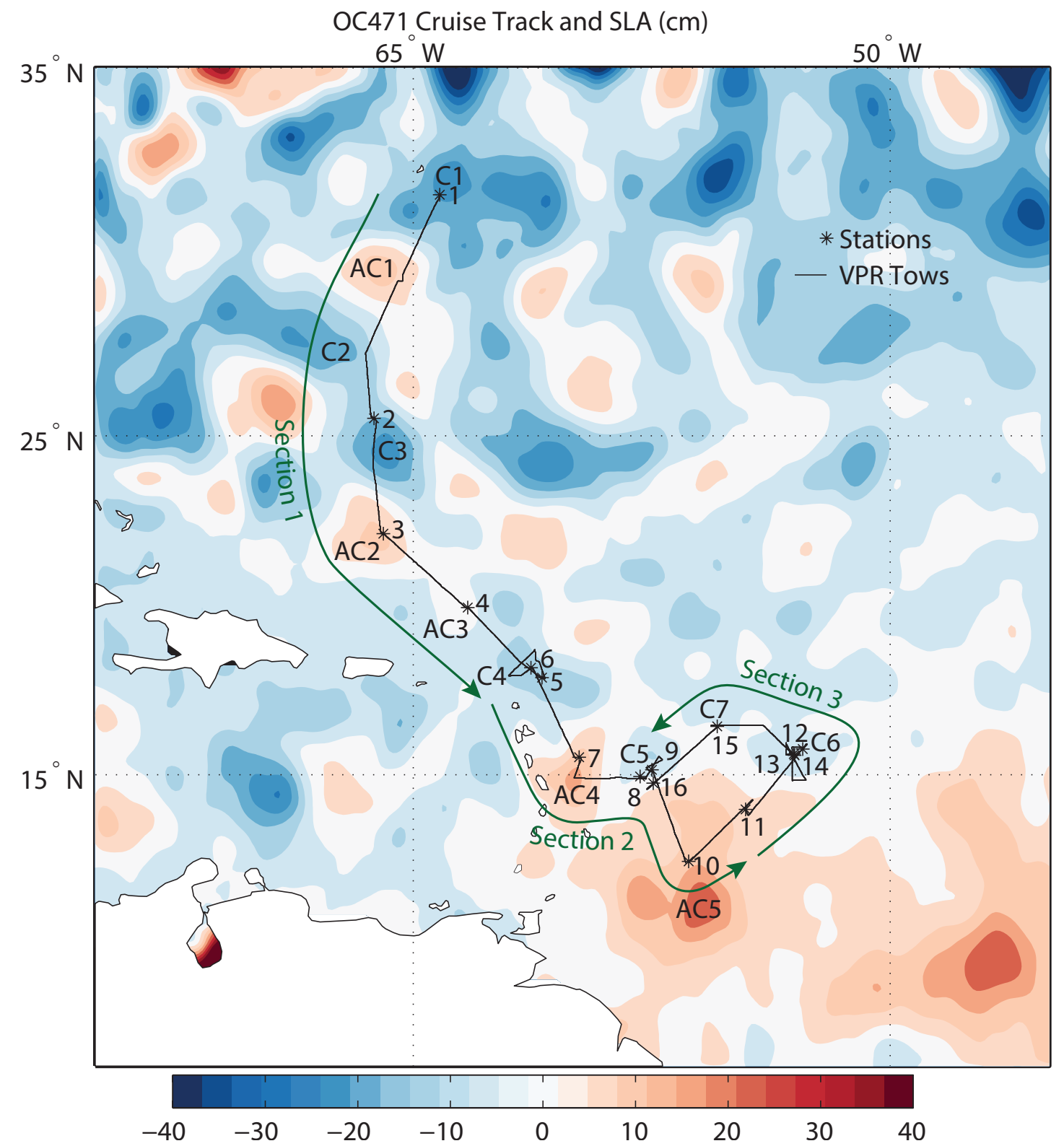

Figure 3-3: Locations of stations and VPR tows for cruise OC471 with sea level anomaly. Black lines indicate VPR tows. Stars indicate station locations. Targeted cyclones and anticyclones are labeled with prefixes "C" and "AC", respectively. Labeled sections are referred to in subsequent analysis. 
collected images (see Chapter 2). Abundances were re-scaled accordingly. For both cruises, each machine-classified image was manually checked and reclassified as necessary, into the three Trichodesmium morphological categories, puff, raft, and bowtie, and an "other" category containing everything else. Trichodesmium puff, raft, and bowtie colonies are readily sampled using the VPR system due to their macroscopic size, but individual trichomes are not distinguishable from other similarly shaped bodies.

The volume of the field of view of was calibrated before each cruise by the tethered copepod method described by Davis et al. (2005) and was $6.929 \mathrm{~mL}$ and $15.168 \mathrm{~mL}$ for the fall and spring cruises, respectively. These volumes are used to convert observation frequencies to abundances. The corresponding vertical and horizontal dimensions of the field of view were $13 \mathrm{~mm}$ x $13 \mathrm{~mm}$ and $12 \mathrm{~mm}$ x $12 \mathrm{~mm}$; the camera and strobe were oriented to produce a deeper and narrower field of view for the spring cruise. Abundance data were gridded to bins $5 \mathrm{~m}$ deep and 11 and $33 \mathrm{~km}$ wide for the fall and spring cruises, respectively. Within each bin, abundances were estimated under the assumption of a Poisson distribution, based on the total water volume imaged and the number of positive identifications.

In addition to imaging, the VPR contains a CTD probe that recorded pressure, temperature, salinity, photosynthetically active radiation (PAR), oxygen, and fluorescence. Physical and bio-optical data from the VPR were binned to the same horizontal resolution as the abundance data and either 1 or $5 \mathrm{~m}$ vertically, as indicated. Advantages of using the VPR include the noninvasive nature of its sampling method and high spatial resolution facilitating observation of phenomena occurring at the mesoscale and even submesoscale.

At each station in between VPR tows, CTD casts measured temperature, salinity and PAR. Water samples collected at depths of 700, 500, 300, 200, 100, 80, 60, 40, $20 \mathrm{~m}$, and the surface were filtered and preserved for nutrient analysis. In the upper $80 \mathrm{~m}$, water samples were gravity filtered and preserved for microscopic enumeration of both Trichodesmium colonies and free trichomes. Microscopic colony counts were 
compared to VPR colony abundances to confirm the calibration of the VPR sampling volume and the image subsampling method applied to the spring cruise (see Chapter 2).

Throughout the cruise, underway sensors measured near-surface environmental conditions. Currents were measured by ADCP. Wind speeds were measured by sensors mounted at $15 \mathrm{~m}$ above sea level on the port and starboard sides of the ship. Temperature profiles were recorded using XBTs deployed approximately every hour while underway.

Sea level anomaly (SLA) along the cruise tracks was estimated by linear interpolation from objectively analyzed fields. The objective analysis combines altimetric data from Envisat, Jason-1 and Jason-2. Eddies were tracked in time and space by visually comparing daily objectively analyzed SLA fields.

\subsubsection{Model}

\section{Experimental Design}

We designed a series of four simulations, two of cyclones and two of anticyclones. For each eddy type, two surface stress formulations were tested. In one, surface stress was calculated based solely on surface wind velocities. In the other, more realistic parameterization, surface stress was related to the relative velocity between air and sea at the interface. Buoyant colonies were represented in these simulations by a tracer with an imposed upward velocity. This experimental design allowed us to analyze the extent to which spatial variation in wind stress due to eddy surface velocities could drive concentration and dilution of buoyant colony concentrations in eddies.

\section{Model Configuration}

Each simulation contained an isolated eddy within a doubly periodic domain. The simulations were constructed based on those developed by Ledwell et al. (2008). The model was the Los Alamos Parallel Ocean Program (POP) version 2, a 3-dimensional 
primitive equation model employing the hydrostatic and Boussinesq approximations (Smith et al., 2000). Horizontal mixing of momentum and tracers was Laplacian with diffusivity and kinematic viscosity of $1 \mathrm{~m}^{2} \mathrm{~s}^{-1}$. The model included k-profile vertical mixing with a background diffusivity of $10^{-5} \mathrm{~m}^{2} \mathrm{~s}^{-1}$. Tracer advection was by a centered difference scheme (MPDCD) with a flux limiter to prevent negative concentrations. Simulations were carried out on an f-plane, at $21^{\circ} \mathrm{N}$, to avoid westward translational motion of the eddies within their domains due to the variation in planetary vorticity with latitude.

The vertical grid contained 72 levels with resolution increasing from $125 \mathrm{~m}$ at 4462 $\mathrm{m}$ depth to $5 \mathrm{~m}$ at the surface. Horizontally, the doubly periodic domain contained $141 \times 141$ cells with zonal and meridional resolution of approximately $3.1 \mathrm{~km}$.

We used the POP version 2 free surface boundary condition. Restoring freshwater and heat fluxes at the surface were applied on timescales of 1 day. Wind stress was formulated as in Ledwell et al. (2008), with parameterizations for uniform and surface current-dependent stress. Wind speed was constant at $4.7 \mathrm{~m} \mathrm{~s}^{-1}$, rotating in direction on a 64 hour cycle. The chosen wind speed was the average wind speed observed during the fall cruise for the period October 9-15, 2010, when the ship was near the region where the association between Trichodesmium colonies and cyclones was observed.

As in Ledwell et al. (2008), hydrographic profiles from the center and periphery of observed eddies were used to initialize the model temperature and salinity fields (Figure 3-4). The eddies simulated were C3 and AC3 from the fall cruise (Figure 3-2). Temperature and salinity fields were initialized based on three locations, the center of eddy C3, the edge between eddies C3 and A3, and the center of eddy A3. A station was located at the center of eddy C3, so CTD measurements were available there to $700 \mathrm{~m}$. CTD temperature and salinity measurements from the VPR were available in the upper $150 \mathrm{~m}$ at the other two locations. Where available, direct CTD observations from the VPR or rosette were used. In other locations, XBT temperature measurements were available to $700 \mathrm{~m}$. Where XBT temperature 
measurements were available but no salinity measurements were taken, salinity was inferred based on the temperature-salinity relationship observed at station 8 , at the center of eddy C3. Below 700m, where neither temperature or salinity measurements were made, climatological values based on WOA2009 were used. Based on center and edge profiles, temperature and salinity were interpolated with a Gaussian transition with an e-folding scale of $68 \mathrm{~km}$. This results in maximum azimuthal velocities at a radius of $48 \mathrm{~km}$. Initial velocity fields were calculated based on geostrophic and cyclostrophic balance with zero motion at the lowermost model level. Whereas addition of a barotropic velocity component brought velocity fields into agreement with observations in Ledwell et al. (2008), such an addition was not required here. Velocity structure is characterized by solid body rotation in the eddy core transitioning to exponential decay with increasing distance from the eddy center. Maximum velocities were $0.22 \mathrm{~m} \mathrm{~s}^{-1}$ for $\mathrm{C} 3$ and $0.41 \mathrm{~m} \mathrm{~s}^{-1}$ for $\mathrm{A} 3$.

Trichodesmium colony buoyancy was simulated by imposing an upward vertical tracer velocity at every location except the surface boundary. This velocity was fixed at $1.2 \times 10^{-4} \mathrm{~m} \mathrm{~s}^{-1}\left(10 \mathrm{~m} \mathrm{~d}^{-1}\right)$. Buoyant Trichodesmium colonies have been observed to rise at velocities greater than $0.001 \mathrm{~m} \mathrm{~s}^{-1}\left(90 \mathrm{~m} \mathrm{~d}^{-1}\right)$ (Walsby, 1992). However, a large range of velocities may be measured in a given location, some positive and some negative. In one study (Walsby, 1978), 69\% of T. thiebautii (puff) colonies, $87 \%$ of T. contortum (raft) colonies, and $100 \%$ of T. erythraeum colonies were positively or neutrally buoyant. Puff colonies were identified as T. thiebautii, whereas rafts were identified as T. contortum if the filaments at the edges were splayed or T. erythraeum if the edges came to a narrow point. In one net tow at $20 \mathrm{~m}$, Walsby (1978) measured floating velocities of $T$. thiebautii (puff) colonies with a range of $\pm 0.7 \mathrm{~mm} \mathrm{~s}^{-1}$ and a mean velocity of $-0.03 \mathrm{~mm} \mathrm{~s}^{-1}$. Mean observed raft velocities are not quoted, but it seems reasonable to assume they are positive, based on the greater proportion of floating raft colonies compared to puff colonies, and that they are less than the maximum velocity observed, $0.001 \mathrm{~ms}^{-1}$. Based on these bounds, a model tracer velocity of $1.2 \times 10^{-4} \mathrm{~m} \mathrm{~s}^{-1}$ was chosen. 
The tracer initial condition consisted of constant tracer concentration throughout the upper $20 \mathrm{~m}$ of the model domain. As the initial velocity fields are only approximate, an initial adjustment of velocities and density structure takes place over the first day of the model run. To avoid influence of this initial adjustment period on the tracer field, the tracer concentration was reset on day 8 of the simulations and the model restarted. Therefore, in the results that follow, time is referenced to the restart day, with day referring to the first day of the restart rather than the day on which the physical initial conditions were initialized. As a result, maximum azimuthal eddy velocities on day 1 differ from those calculated in the initial conditions, changing from 0.22 to $0.23 \mathrm{~m} \mathrm{~s}^{-1}$ in the cyclone with eddy-wind interaction and to $0.24 \mathrm{~m} \mathrm{~s}^{-1}$ in the cyclone without eddy-wind interaction. Azimuthal velocities decayed from -0.41 to $-0.33 \mathrm{~m} \mathrm{~s}^{-1}$ in the anticyclone with eddy-wind interaction and to -0.34 in the anticyclone without eddy-wind interaction.

\subsection{Results}

\section{Trichodesmium Observations, SLA, and Hydrography}

During the fall and spring cruises, two contrasting patterns of Trichodesmium association with eddies were encountered. For the spring cruise as a whole, the correlation coefficient between sea level anomaly and depth-integrated colony abundance was 0.44 with a p-value of $7 \times 10^{-24}$ when including data from all VPR transects. This reflects a tendency for greater Trichodesmium abundances in anticyclones. For the fall cruise the correlation coefficient and p-value were -0.38 and $1 \times 10^{-7}$, reflecting elevated abundance in cyclones. Thus, these contrasting associations with the eddy field explained a small part of the observed variability in integrated abundance. However, within localized regions on each cruise, the eddy-correlated patterns were stronger. 

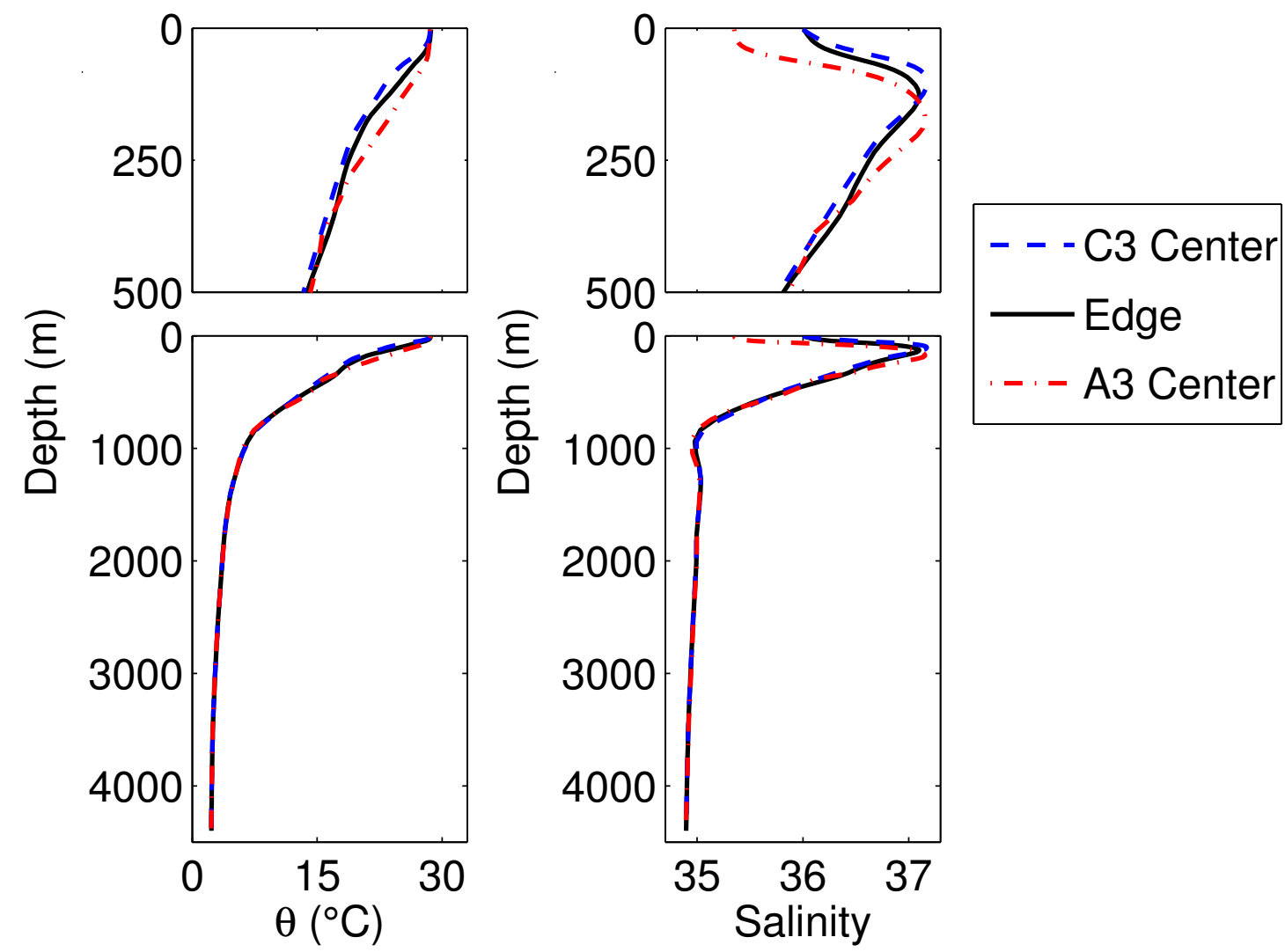

Figure 3-4: Potential temperature and salinity profiles used to initialize eddy models.

\subsubsection{Fall 2010: Enhanced Abundance of Trichodesmium Colonies in Cyclones}

The fall cruise (OC469) transected a roughly $700 \mathrm{~km}$ region (Section 2 in Figure 3-2) where Trichodesmium colony abundance patterns reflected variations in sea level anomaly associated with the eddy field. Here, in contrast to both the Spring 2011 cruise and previous observations in the subtropical North Atlantic (Davis and McGillicuddy, 2006), Trichodesmium colony abundances were locally elevated in cyclones and nearly absent outside of them. Over this region (Section 2), the correlation coefficient between interpolated sea level anomaly and integrated Trichodesmium abundance was -0.67 with a p-value of $2 \times 10^{-10}$. Surface abundances within the two cyclones (C3 and $\mathrm{C}$ in Figure 3-5) fell in the range of $0.2-0.7$ colonies $\mathrm{L}^{-1}$, whereas in the anticyclones (AC3 and $\mathrm{AC} 4)$ colony abundances near the surface ranged from 
below the limit of detection to 0.2 colonies $\mathrm{L}^{-1}$. Abundances within cyclones and anticyclones in this region were low compared to surface colony abundances observed elsewhere on the cruise, averaging 0.8 colonies $\mathrm{L}^{-1}$ to the north and 2.0 colonies $\mathrm{L}^{-1}$ to the south of this section. Rafts were the dominant morphology here and were vertically distributed differently from the other morphologies with greatest abundance within the surface mixed layer. On the southwest side of the center of eddy C3, integrated colony abundances in the closest four 11-km grid cells to the center averaged $12.7 \times 10^{3}$ colonies $\mathrm{L}^{-1}$. Integrated colony abundances in the nine $11-\mathrm{km}$ grid cells centered in eddy A3 averaged $0.76 \times 10^{3}$ colonies $\mathrm{L}^{-1}$.

At the time of sampling, C3 and A3 had similar diameters of roughly $150 \mathrm{~km}$ based on the largest closed SLA contours encircling the eddies. Although the eddies were not symmetric in shape, a representative radius at which maximum velocities were found was approximately $50 \mathrm{~km}$ for both eddies. Typical maximum velocities at approximately $20-30 \mathrm{~m}$ depth were $0.13-0.45 \mathrm{~m} \mathrm{~s}^{-1}$ for eddy $\mathrm{C} 3$ and $0.35-0.6 \mathrm{~m} \mathrm{~s}^{-1}$ for eddy A3 based on shipboard ADCP measurements. 


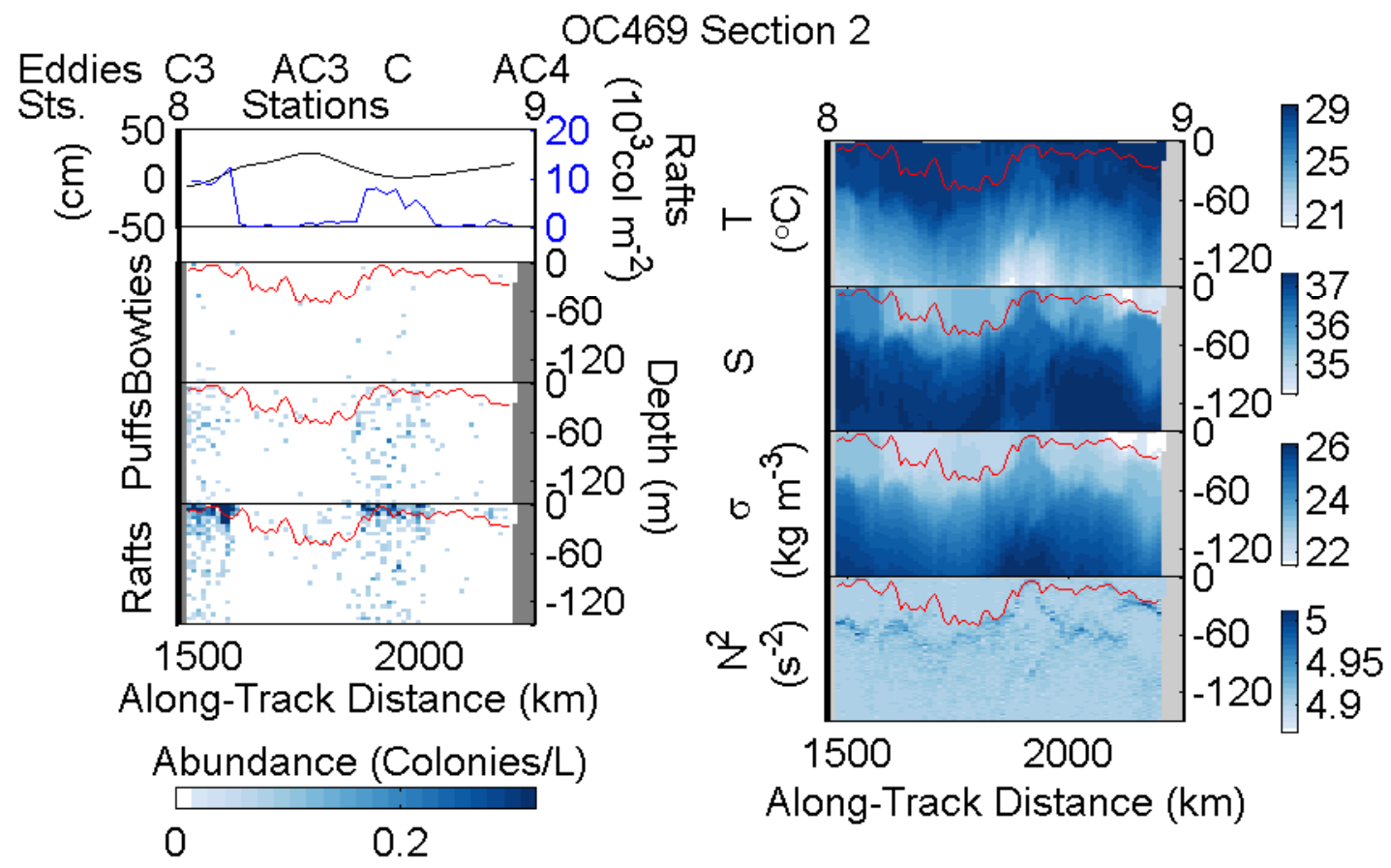

Figure 3-5: Trichodesmium abundance and physical properties associated with eddies transected on section 2 of fall 2010 cruise OC469. Black line in upper panel is sea level anomaly $(\mathrm{cm})$. Blue line in upper panel represents integrated raft colony abundance $\left(10 \times 10^{3}\right.$ colonies $\left.\mathrm{m}^{-2}\right)$. Red lines show mixed layer depth as determined by a threshold criterion of $0.125 \mathrm{~kg} \mathrm{~m}^{-3}$ density difference from the surface. Right panels (top to bottom) display temperature, salinity, density, and buoyancy frequency. 


\subsubsection{Conceptual Model}

We have hypothesized that convergence (divergence) due to the modification of wind stress by cyclone (anticyclone) ocean surface velocities could lead to concentration (dilution) of buoyant Trichodesmium colonies. We first explore this mechanism with an idealized analytical model. We derive equations for the Ekman transport assuming Gaussian velocity structure in a cyclonic or anticyclonic eddy. The idealized surface velocity field reflects solid body rotation at the eddy center and exponential decay far from the eddy center as in Martin and Richards (2001). The purely azimuthal velocity is described by

$$
\mathbf{v}_{\theta}(r)=V \frac{r}{R} \exp \left[\frac{1}{2}\left(1-\frac{r^{2}}{R^{2}}\right)\right]
$$

where $V$ is the maximum azimuthal velocity, which occurs at a radius $r=R$ from the eddy center. In the northern hemisphere, $V$ is positive in a cyclone and negative in an anticyclone.

The steady Ekman transport is given by,

$$
\mathbf{U}_{\mathbf{E}}=\left(U_{E}, V_{E}\right)=\left(\frac{\tau^{(y)}}{\rho_{0} f},-\frac{\tau^{(x)}}{\rho_{0} f}\right)
$$

(see Batchelor (1967) equation 4.4.II), where $\rho_{0}$ is the density of seawater $\left(1026 \mathrm{~kg} \mathrm{~m}^{-3}\right)$, and $f$ is the Coriolis parameter $\left(5.21 \times 10^{-5} \mathrm{~s}^{-1}\right) . \tau^{(x)}$ and $\tau^{(y)}$ are the zonal and meridional components of the wind stress at the ocean surface.

We use the same wind stress parameterization as Dewar and Flierl (1987):

$$
\tau=\rho_{a} c_{d}\left|\mathbf{U}_{\mathbf{a}}-\mathbf{u}\right|\left(\mathbf{U}_{\mathbf{a}}-\mathbf{u}\right),
$$

where $\rho_{a}$ is the density of air $\left(1.2 \mathrm{~kg} \mathrm{~m}^{-3}\right)$; $c_{d}$ is the drag coefficient (0.00118); $\mathbf{U}_{\mathbf{a}}$ is the wind velocity; and $\mathbf{u}$ is the sea surface velocity. We suppose the wind is westerly and uniform $\left(\mathbf{U}_{\mathbf{a}}=U_{a} \hat{\mathbf{1}}\right.$, where $\hat{\mathbf{1}}$ is a unit vector in the zonal direction). We define $u_{0}$ and $v_{0}$ as the zonal and meridional components of the azimuthal sea surface velocity 
associated with the eddy ( $\mathbf{v}_{\theta}$, Equation 3.1). As in Dewar and Flierl (1987), we assume that eddy surface velocities are small compared to wind velocity $\left(|u| \ll\left|U_{a}\right|\right)$, so that terms that are second order in $u_{0}$ and $v_{0}$ can be ignored. Also, we assume that the velocity due to the wind stress is small compared to the eddy velocity. The wind stress equation becomes

$$
\tau=\rho_{a} c_{d}\left(\left(U_{a}^{2}-2 U_{a} u\right) \hat{\mathbf{i}}-U_{a} v \hat{\mathbf{j}}\right),
$$

where $\hat{\mathbf{i}}$ and $\hat{\mathbf{j}}$ are unit vectors in the $\mathrm{x}$ (east) and $\mathrm{y}$ (north) directions, respectively.

The zonal and meridional components of the Ekman transport are therefore

$$
\begin{aligned}
& U_{E}=-\frac{\rho_{a} c_{d}}{\rho_{0} f} U_{a} v_{0} . \\
& V_{E}=-\frac{\rho_{a} c_{d}}{\rho_{0} f}\left(U_{a}^{2}-2 U_{a} u_{0}\right),
\end{aligned}
$$

The first term contributing to $V_{E}$ (Equation 3.5b) is the wind stress in the absence of sea surface motion. The second term in $V_{E}$ and the single term contributing to $U_{E}$ (Equation 3.5a) are due to modification of the wind stress by the sea surface velocity.

Now, we make a series of assumptions. We assume that velocities are uniform over a surface layer of depth $H$, and that tracer is initially uniformly distributed throughout this layer. We further assume that over time, the tracer remains in this upper layer, although it maybe redistributed horizontally. The velocity in this layer is the surface velocity $\mathbf{u}$, with components $u$ and $v$, which is the sum of the azimuthal eddy velocity and the Ekman transport divided by the layer depth.

$$
\begin{aligned}
& u=u_{0}+u_{E}=-v_{\theta} \sin \theta-\frac{\rho_{a} c_{d}}{\rho_{0} f H} U_{a} v_{0} \\
& v=v_{0}+v_{E}=v_{\theta} \cos \theta-\frac{\rho_{a} c_{d}}{\rho_{0} f H}\left(U_{a}^{2}-2 U_{a} u_{0}\right)
\end{aligned}
$$

In a cyclone, trajectories originating close to the eddy center are trapped within the 
eddy, but slowly spiral inward toward the center as the center region converges (Figure 3-6).
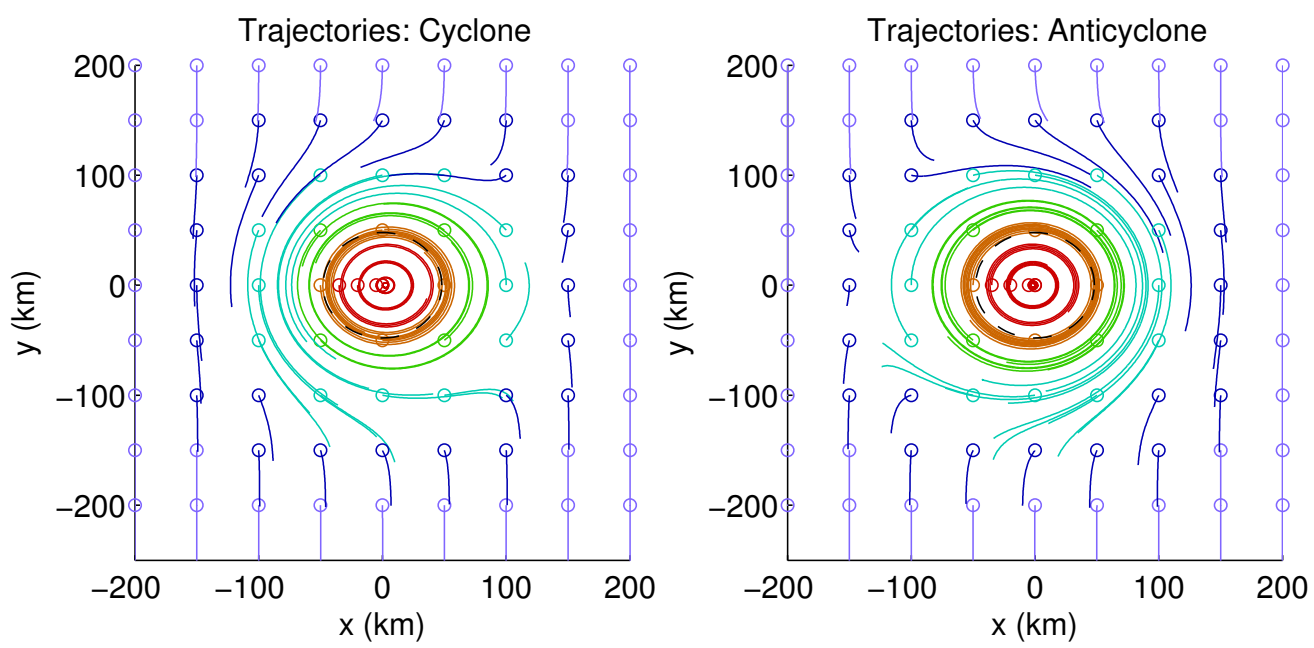

Figure 3-6: 30-day trajectories calculated based on surface velocities resulting from a combination of azimuthal eddy velocities and wind-driven motion (Equation 3.6). A black dashed line marks the radius of maximum velocity of the eddy at $48 \mathrm{~km}$. A circle represents the point of origin of each trajectory. 
The evolution of tracer concentration, $C$, over time along a trajectory can be described by

$$
\frac{1}{C} \frac{D C}{D t}=-\nabla \cdot \mathbf{u}_{\mathbf{E}}-\frac{1}{C} A_{H} \nabla^{2} C
$$

where $\nabla \cdot \mathbf{u}_{\mathbf{E}}$ is the divergence of the horizontal velocity field (the azimuthal eddy velocity is non divergent) and $A_{H}$ is the coefficient used to parameterize horizontal mixing. Along a trajectory, changing tracer concentration is controlled by horizontal divergence of the velocity field, which will tend to produce gradients in tracer concentration, and diffusive processes which will smooth those gradients.

We are interested in the overall change in tracer concentration over the eddy core, which we will define as the region within the radius of maximum velocity, $r<R$. The horizontal velocity divergence is equal to the Ekman pumping, $w_{e}$,

$$
w_{e}=\frac{\rho_{a}}{\rho_{0} f_{0}} c_{d} U_{a}\left(2\left(u_{E}\right)_{y}-\left(v_{E}\right)_{x}\right)
$$

(Dewar and Flierl, 1987).

Under the same assumptions as before, we can estimate the flux of water into an eddy as the opposite of the horizontal velocity divergence integrated over the eddy center.

$$
F=\frac{\rho_{a}}{\rho_{0} f_{0}} c_{d} U_{a} 3 \pi R V
$$

At time $t=0$, we assume the Trichodesmium colonies are spread uniformly throughout the surface layer with a concentration $C_{0}$. Therefore, the initial flux of colonies into the eddy center is given by $\phi=C_{0} F$.

At time zero, the fractional rate of change of integrated abundance within the eddy is:

$$
\frac{\mathrm{d} C_{1}}{\mathrm{~d} t}=\frac{3 \rho_{a} c_{d} C_{0} U_{a} V}{\rho_{0} f R H}
$$

Using parameters from Table 3.1, the initial relative rate of increase of concentra- 


\begin{tabular}{llll}
\hline Description & Variable & Value & Units \\
\hline Density, air & $\rho_{a}$ & 1.2 & $\mathrm{~kg} \mathrm{~m}^{-3}$ \\
Density, seawater & $\rho_{0}$ & 1026 & $\mathrm{~kg} \mathrm{~m}^{-3}$ \\
Coriolis parameter, $21^{\circ} \mathrm{N}$ & $f$ & $5.21 \times 10^{-5}$ & $\mathrm{~s}^{-1}$ \\
Drag coefficient & $c_{d}$ & $1.18 \times 10^{-3}$ & \\
Surface wind speed & $U_{a}$ & 4.7 & $\mathrm{~m} \mathrm{~s}^{-1}$ \\
Eddy radius of maximum velocity & $R$ & 48 & $\mathrm{~km}^{-1}$ \\
Maximum azimuthal velocity, cyclone & $V$ & 0.222 & $\mathrm{~m} \mathrm{~s}^{-1}$ \\
Maximum azimuthal velocity, anticyclone & $V$ & -0.408 & $\mathrm{~m} \mathrm{~s}^{-}$ \\
Mixed layer depth & $H$ & 30 & $\mathrm{~m}$
\end{tabular}

Table 3.1: Constants and eddy characteristics

tion within the center of the cyclone is $0.5 \%$ per day. The initial rate of decrease in an anticyclone is $1.0 \%$ per day.

Over time, additional factors will influence the flux of tracer into the eddy. The tracer concentration itself will develop spatial variability, which complicates the calculation of the tracer flux in this simple model. As gradients in tracer concentration develop, diffusive processes will counteract them. Tracer concentrations in water entering the eddy center may in time exhibit the effects of the convergence/divergence tendency outside the eddy, which is opposite that in the center.

Thus, many factors not represented in this simplified analytical model could be simulated more realistically in a numerical model. These include the effects of continuous and evolving spatial variability in the tracer distribution. Additionally, there will be small differences due to the neglected higher order terms in the wind stress formulation (Equations 3.3 and 3.4). Further, small changes might be expected due to the modification of sea surface velocities due to the Ekman transport. Buoyant tracer distributions in more realistic simulations of cyclonic and anticyclonic eddies are presented next in Section 3.4.3.

\subsubsection{Numerical Model}

The eddies with eddy-wind interaction decayed throughout the 50-day simulation with maximum azimuthal geostrophic velocities decreasing from roughly 0.23 to 0.17 
$\mathrm{m} \mathrm{s}^{-1}$ in the cyclone and -0.33 to $-0.25 \mathrm{~m} \mathrm{~s}^{-1}$ in the anticyclone. In the absence of eddywind interaction decay was much slower, with maximum eddy velocities remaining roughly constant at $0.241 \mathrm{~m} \mathrm{~s}^{-1}$ in the cyclone and $0.35 \mathrm{~m} \mathrm{~s}^{-1}$ in the anticyclone from days 1 to 50. In all four cases, the mean mixed layer depth deepened over the course of the simulations, from initial conditions with weak surface stratification to a mixed layer of approximately $30 \mathrm{~m}$ by day 50 .

Time-mean cross sections reveal the impact of eddy-wind interactions on simulated tracer distributions (Figure 3-7). Surface tracer concentrations increased in the cyclone and decreased in the anticyclone both with and without eddy-wind interaction. In the cyclone, depth-integrated tracer concentrations increased in the center of the cyclone with eddy-wind interaction but remained spatially uniform in the simulations without eddy-wind interaction. In the anticyclone, integrated tracer concentrations decreased in the eddy center both with and without eddy-wind interaction, but the decrease was more extreme in the simulations with eddy-wind interaction.

In the cyclone simulation without eddy-wind interaction, mixed layer depth was shallower inside the core of the cyclone than outside it. Therefore, buoyant tracer mixed vertically over a reduced volume inside the cyclone, producing a local surface tracer maximum above the center of the eddy (Figure 3-7). However, integrated tracer concentrations were roughly constant throughout the model domain. Flux into the eddy across a circle of radius of $51 \mathrm{~km}$ (slightly greater than the radius associated with maximum eddy velocity) was nearly zero throughout the upper 50 meters (Figure $3-8)$.

Compared to the cyclone without eddy-wind interaction, the cyclone eddy-wind interaction exhibited a reduction in doming of the pycnocline (Figure 3-7). This flattening of isopycnals results from downward motions within the eddy due to convergent Ekman transport. The inward volume flux associated with these downward motions is concentrated in the upper $25 \mathrm{~m}$ (Figure 3-8). This inward transport leads to concentration of the buoyant tracer near the cyclone center. Because this tracer signal results from horizontal convergence rather than a reduction in dilution by vertical 
mixing, it is associated with a maximum in integrated tracer concentration (Figure 3-7, lower left).

The anticyclone simulated without eddy-wind interaction exhibits a deeper mixed layer at eddy center, contributing to a reduction in surface tracer concentration through dilution. However, in contrast to the corresponding cyclonic simulation, integrated tracer concentration decreases at the eddy center. Thus, there is horizontal divergence of tracer as well as dilution through deeper mixing. In fact, there is a net volume flux out of the eddy in the upper $20 \mathrm{~m}$, nearly balanced by flux into the eddy between 20 and $40 \mathrm{~m}$ (Figure 3-8). Due to imposed tracer buoyancy, concentrations increase toward the surface and therefore more tracer is transported out of the eddy in the upper layer than is transported in through the lower layer. This divergence of depth-integrated tracer concentration at the center of the anticyclone in the absence of eddy-wind interaction was associated with upwelling near the base of the mixed layer that persisted throughout the model run. This upwelling had a maximum mean magnitude over the eddy core $(r<48 \mathrm{~km})$ of $2.9 \times 10^{-4}$ at $23 \mathrm{~m}$ depth.

When eddy-wind interaction is included in the anticyclonic eddy simulation, the mixed layer depth within the anticyclone is reduced, particularly at the center. This reduction in mixed layer depth is due to upward velocities induced by divergent Ekman transport (Figure 3-7). Compared to the anticyclone without eddy-wind interaction, outward flow in the upper $20 \mathrm{~m}$ is increased and inward flow in the 20$40 \mathrm{~m}$ depth interval is reduced (Figure 3-8). The accompanying reduction in tracer concentration leads to an even greater reduction in integrated tracer concentration in the eddy core than in the simulation without eddy-wind interaction (Figure 3-7, bottom right).

We assess the rate of accumulation or dispersion of tracer as the relative difference in mean integrated concentrations within the core $(r<48 \mathrm{~km})$ from that at the edge of the model domain, which remains constant throughout the run and is thus also representative of the initial concentration throughout the domain (Figure 3-9). In the cyclone without eddy-wind interaction, this difference is negligible throughout 


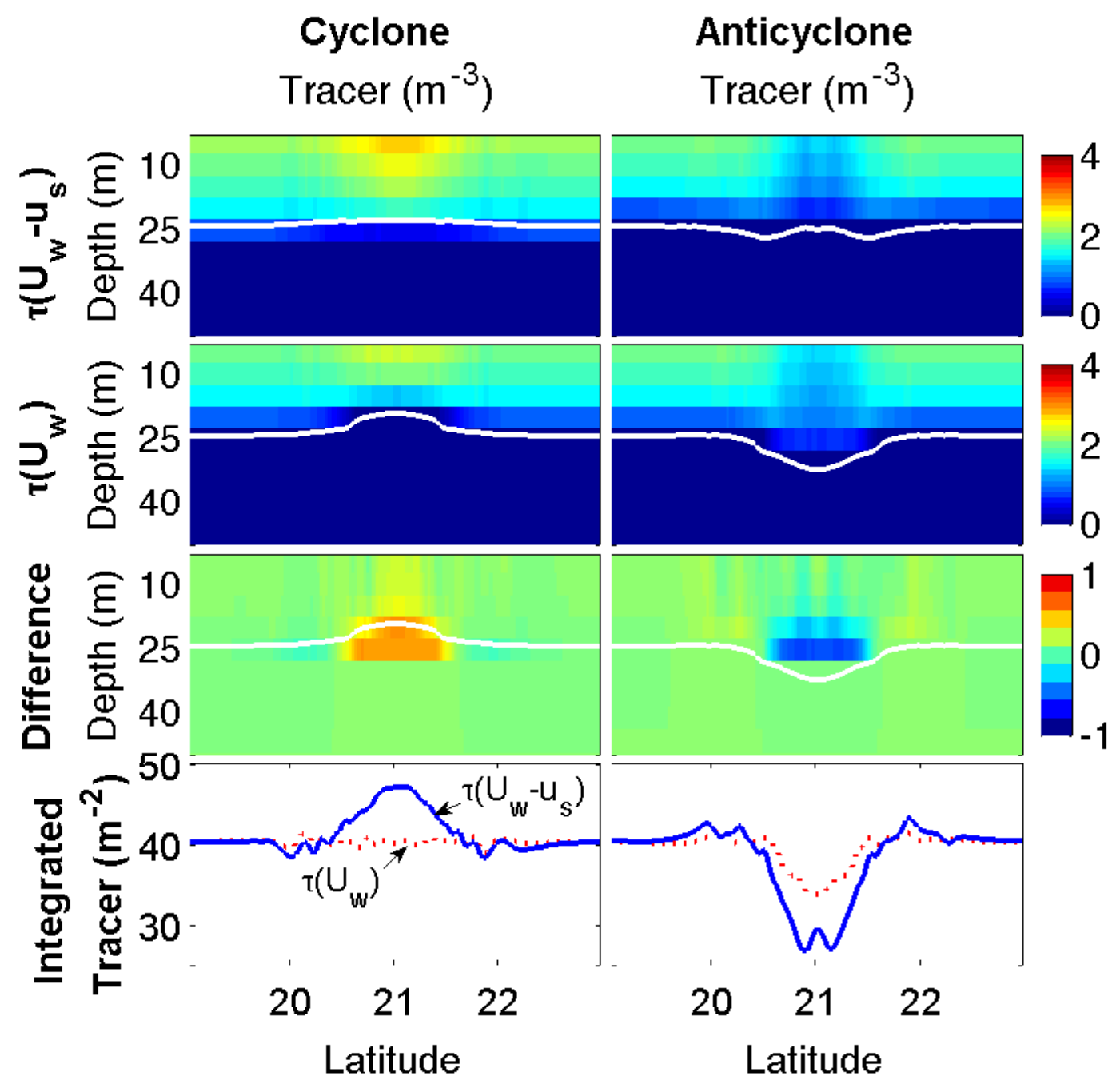


Figure 3-7: 20-day (days 12 to 31) mean tracer concentrations. Upper six panels display tracer concentration fields in cross-sections through eddy center with white contours marking mixed layer depths determined by a $0.025 \mathrm{~kg} \mathrm{~m}^{-3}$ potential density difference from the surface. Top: model runs including wind stress as a function of wind and sea surface velocity $\left(\tau\left(\mathbf{U}_{\mathbf{w}}-\mathbf{u}_{\mathbf{s}}\right)\right)$. Second row: model runs including wind stress as a function of wind velocity only $\left(\tau\left(\mathbf{U}_{\mathbf{w}}\right)\right)$. Third row: difference between model runs with and without eddy-wind interaction with mixed layer depths from the run without eddy-wind interaction. Bottom: depth-integrated tracer concentrations for the cross-sections displayed $\mathrm{n}$ rows 1 and 2. Blue line is based on model with wind stress a function of wind and sea surface velocity. Red dashed line is based on model with wind stress dependent on wind velocity only. In all rows, left panels display cyclone model runs and right panels display anticyclone model runs.

the model run. In the cyclone with eddy-wind interaction, the relative difference increases throughout the run.

In the anticyclone without eddy-wind interaction, there is a modest decrease in integrated tracer concentration at the eddy center associated with the upwelling at the base of the mixed layer. This upwelling also occurs in the model without eddywind interaction but does not occur in the absence of wind forcing altogether. In the anticyclone with eddy-wind interaction, integrated tracer concentrations in the eddy core are diluted at a slightly greater rate than in the case without eddy-wind interaction. As in the cyclone with eddy-wind interaction, the rate of change of concentration in the center of the eddy slows with time.

The initial rate of change of tracer concentration due to the eddy-wind interaction mechanism was predicted for Gaussian eddies with maximum azimuthal velocities consistent with day 1 of the model runs and with maximum azimuthal velocities consistent with the model initial conditions. The estimates based on day 1 azimuthal velocities slightly underestimate the decrease in the anticyclone and overestimate the increase in the cyclone (Figure 3-9). The initial simulated rates of aggregation in the model were 0.50 and $-1.08 \%$ per day for the cyclone and anticyclone, respectively. The rates predicted for a Gaussian eddy with day 1 simulation velocities were 0.58 and -0.75 , whereas the rates predicted for a Gaussian eddy with velocities corresponding to the initial conditions were 0.55 and -1.03 . The difference between dilution rates 


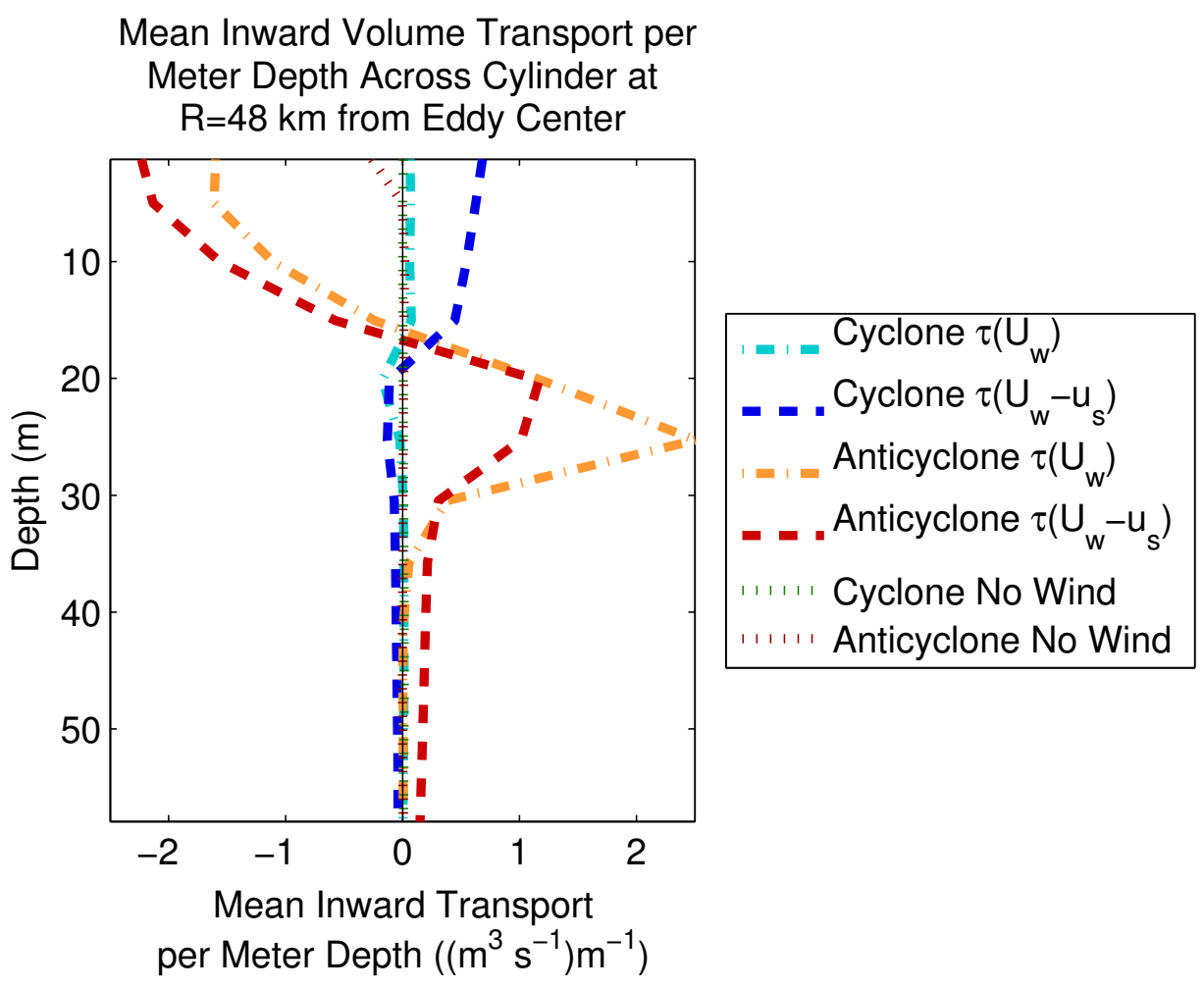

Figure 3-8: 20-day mean inward volume transport per meter depth across a circle centered at eddy center with walls at a radius of $51 \mathrm{~km}$, just beyond the radius of maximum eddy velocity. Volume transports are displayed for six simulations: cyclone without eddy-wind interaction, cyclone with eddy-wind interaction; anticyclone without eddy-wind interaction; anticyclone with eddy-wind interaction, cyclone without wind, anticyclone without wind.

for the anticyclone with and without eddy-wind interaction was $-0.75 \%$ per day, consistent with the value predicted based on day 1 simulation velocities.

The slowing of the rate of change of tracer concentration over time is due to a combination of factors. In part, it can be explained by the decaying eddy velocities, which are accompanied by a reduction in the divergent component of the Ekman transport. As discussed by Dewar and Flierl (1987), the eddy-wind interaction mechanism ("top drag") contributes to eddy decay. Total kinetic energy in all simulations decayed over time (Figure 3-10). Kinetic energy in the simulations including the eddy-wind interaction mechanism decayed more than four times as quickly than in the simulations with wind stress but without eddy-wind interaction. 
Another contributing factor is down-gradient diffusive flux as tracer gradients grow over the course of the simulation. This contribution is evaluated by comparing the contribution to concentration change due to divergence of the velocity field $\left(\nabla \cdot \mathbf{u}_{\mathbf{E}}\right)$ with the counteracting contribution due to diffusive processes $\left(\frac{1}{C} A_{H} \nabla^{2} C\right)$. Based on the analytical cyclone velocity field, the maximum contribution by divergence is $9.9 \times 10^{-8} \mathrm{~s}^{-1}$. For the purposes of estimating the diffusive component that might be associated with the length scale and abundance distribution in such an eddy, we use a diffusivity of $A_{H}=200 \mathrm{~m}^{2} \mathrm{~s}^{-1}$ at a scale of $100 \mathrm{~km}$, based on Okubo (1971). This is an "effective" diffusivity representing sub-eddy-scale turbulent transports. The magnitude of $\nabla^{2} C$ is estimated as twice the tracer difference from center to twice the radius of maximum velocity, $R$, divided by the square of $2 R(2 R \sim 100$ $\mathrm{km})$. The approximate compensating contribution by diffusive processes based on the tracer field in the numerical solution is $-9.2 \times 10^{-9} \mathrm{~s}^{-1}$ on day 55 of the simulation and $-1.9 \times 10^{-8} \mathrm{~s}^{-1}$ on day 180 . In the anticyclone, the maximum contribution to tracer concentration change due to divergence of the analytical velocity field is $-1.4 \times 10^{-7} \mathrm{~s}^{-1}$. The approximate compensating diffusive contribution is $2.7 \times 10^{-8} \mathrm{~s}^{-1}$ on day 55 and $9.5 \times 10^{-8} \mathrm{~s}^{-1}$ on day 180 . In addition to the increasing contributions due to diffusive processes, over time the magnitude of divergence of the velocity field is expected to decrease due to eddy decay. Thus, down-gradient diffusive processes have the potential to noticeably impact rates of concentration change over timescales similar to the length of the model run.

On longer timescales, trajectories crossing into the center of the eddy may eventually carry reduced tracer if they originate far enough from the eddy center. However, integration tracer concentration along trajectories (Equations 3.6 and 3.7) in the cyclonic case, ignoring effects of diffusive processes, suggest this effect would not become evident in until after about 140 days, near the end of these simulations. Before that period, the concentration associated with parcels crossing into the center of the eddy would be slowly increasing, in the absence of an opposing diffusive flux. 


\subsubsection{Spring 2011: Trichodesmium Colonies in Anticyclones}

On the spring cruise (OC471), two anticyclones (A4 and A5, Figure 3-11) coincided geographically with local peaks in Trichodesmium colony abundance. The local correlation between sea level anomaly and integrated Trichodesmium abundance was 0.57 with a p-value of $2 \times 10^{-15}$ in the region designated as Section 2 on the spring cruise extending from $18.3^{\circ} \mathrm{N},-61.6^{\circ} \mathrm{W}$, south to $13.0^{\circ} \mathrm{N},-55.6^{\circ} \mathrm{W}$ (Figure $3-11$ ). In this region, abundance patterns were vertically and laterally consistent across morphologies.

Whereas Davis and McGillicuddy (2006) observed elevated colony abundances warm and salty anticyclones, salinity within the anticyclones associated with elevated Trichodesmium abundance on the spring cruise was anomalously low. Based on objectively analyzed of sea level anomaly fields, anticyclone A5 is one of several rings to have been shed into this region by the North Brazil Current (NBC) in spring 2011. Anticyclone A4 appears to have evolved from remnants of an earlier such ring to pass through the area. Its location and history are consistent with a tendency for North Brazil Current rings to deflect northward upon interaction with the Lesser Antilles as described by Fratantoni and Richardson (2006).

The North Brazil Current is one of several flows that entrain and transport Amazon River discharge (Coles et al., 2013; Richardson and Reverdin, 1987). Coles et al. (2013) described an indirect northward pathway for Amazon river discharge transport into the western tropical Atlantic, with approximately $65 \%$ of floats leaving the river mouth between the months of February and June following this pathway. The pathway was characterized by looping trajectories and long residence time in the North Brazil Current region. The NBC retroflection persists through much of the year and sheds rings that carry freshwater emanating from the Amazon River plume to the northwest near the South American continent and into the Caribbean Sea (Coles et al., 2013; Hellweger and Gordon, 2002). However, according to Hellweger and Gordon (2002), the seasonal retroflection is typically observed in the months of June-March. In the absence of the retroflection, Amazon River water is transported 
along this pathway directly through the continuation of the NBC. Plume advection and anticyclonic eddy formation in the North Brazil Current region are evident in HYCOM hindcasts from May 2011, when A4 and A5 were observed; in fact, in the hindcast for the date anticyclone A5 was transected, a large, fresh anticyclone was present at the same location (Figure 3-12). Together, these lines of evidence suggest that the anomalously fresh water encountered in A4 and A5 originated in the Amazon River plume.

Integrated Trichodesmium colony abundances within spring cruise Section 2 were anticorrelated with average salinity and positively correlated with average temperature over the upper $100 \mathrm{~m}$ (Figure 3-13, lower two panels). The local coefficient of correlation of integrated colony abundance with upper $100 \mathrm{~m}$ mean salinity was -0.84 with a p-value of $1 \times 10^{-15}$. The local correlation coefficient of integrated Trichodesmium colony abundance with temperature at $6 \mathrm{~m}$ depth was 0.76 with a p-value of $1 \times 10^{-11}$. Thus, correlations of integrated Trichodesmium abundance with both near-surface temperature and 100-m depth-average salinity were strong in Section 2 of the spring cruise. However, on the scale of the spring cruise as a whole, integrated Trichodesmium abundances reflect patterns in upper water column salinity more closely than temperature (Figure 3-13, upper two panels).

Amazon river outflow has been associated with elevated nutrient concentrations, including phosphorus and iron, in previous studies (Subramaniam et al., 2008; Bergquist and Boyle, 2006; Tovar-Sanchez and SañudoWilhelmy, 2011; Sohm and Capone, 2010). Iron concentrations were not measured in the present study, but phosphorus concentrations were. We therefore investigated the relationship between salinity and phosphorus in the Spring 2011 data to determine if there was evidence of nutrient supply associated with the fresh water that might explain the observed patterns in Trichodesmium abundance (Figure 3-14).

There was not a clear correlation between salinity and phosphate. Phosphate levels at low salinity were typically at or slightly above the mean for each depth measured in the upper $40 \mathrm{~m}$, with the exception of station 16, which had very low salinity at the 
surface as well as very low phosphate. Excess phosphate $\left(\mathrm{P} *=\mathrm{PO}_{4}-\frac{1}{16} \mathrm{NO}_{3}\right.$, where 16:1 is the Redfield ratio of nitrogen to phosphorus in organic matter) was positive in low salinity waters except for station 16. The presence of excess phosphate suggests conditions conducive to Trichodesmium growth. However, iron is also necessary and can limit Trichodesmium growth even when excess phosphate is present.

High levels of phosphate were measured below the low salinity lens at Station 10, at $40 \mathrm{~m}$ depth. One possible explanation for this anomalously high phosphate concentration is that shading due to elevated turbidity associated with biological activity in the low salinity lens may have prevented primary producers from growing in the waters below the lens and drawing down the phosphate concentrations there. Another possible explanation is remineralization below the lens of sinking biological material originating from the lens.

Trichodesmium abundance was compared with mean salinity over the upper $100 \mathrm{~m}$ (Figure 3-13) in order to capture the effect of freshwater influence at station 7 , where the freshwater anomaly was distributed over a deeper mixed layer, leading to a smaller surface anomaly. Similarly, we compare excess phosphorus based on mean phosphate and nitrate in the upper $100 \mathrm{~m}$ with mean salinity in the upper $100 \mathrm{~m}$ (Figure 314, lower right). If station 16, which is anomalous in many respects, is excluded, there appears to be a linear relationship between excess phosphorus in the upper $100 \mathrm{~m}$ and mean salinity in the upper $100 \mathrm{~m}$. However, this relationship is highly dependent on the elevated phosphate concentrations below the freshwater lens at station 10. If the elevated phosphorus there predates the arrival of the freshwater lens and simply has not been drawn down due to shading associated with the lens, then the phosphorus below the lens should be excluded as evidence of riverine nutrient delivery. On the other hand, if the elevated phosphorus below the fresh lens is the result of remineralization of biological material delivered to the region through advection with the lens, then it would make sense to include this phosphate anomaly in analysis of the relationship between phosphate availability and salinity. If the second interpretation is correct, the approximate linear relationship between average excess phosphorus 
and average salinity in the upper $100 \mathrm{~m}$ might be interpreted as evidence of mixing between a fresh, phosphate-rich riverine endmember and a saline, phosphate-poor oceanic endmember.

Trichodesmium alkaline phosphatase activity tends to increase with decreasing salinity (Figure 3-14, lower left). This relationship is consistent with a scenario in which phosphorus supplied to this region through the river plume water is in organic form due to upstream biological activity. Trichodesmium has a demonstrated ability to utilize dissolved organic phosphorus (DOP) (Dyhrman et al., 2006; Orchard et al., 2010), and may rapidly assimilate DOP as it becomes available. However, the Trichodesmium alkaline phosphatase activity data are normalized to number of colonies rather than carbon biomass, so variability in the size of colonies could influence the results. Additionally, the measured Trichodesmium alkaline phosphatase activities include activity due to epibionts associated with the colonies and therefore must be considered representative of the activity of the consortium rather than Trichodesmium alone. 


\section{Percent Difference in Eddy Center Tracer Concentration from Initial Value}

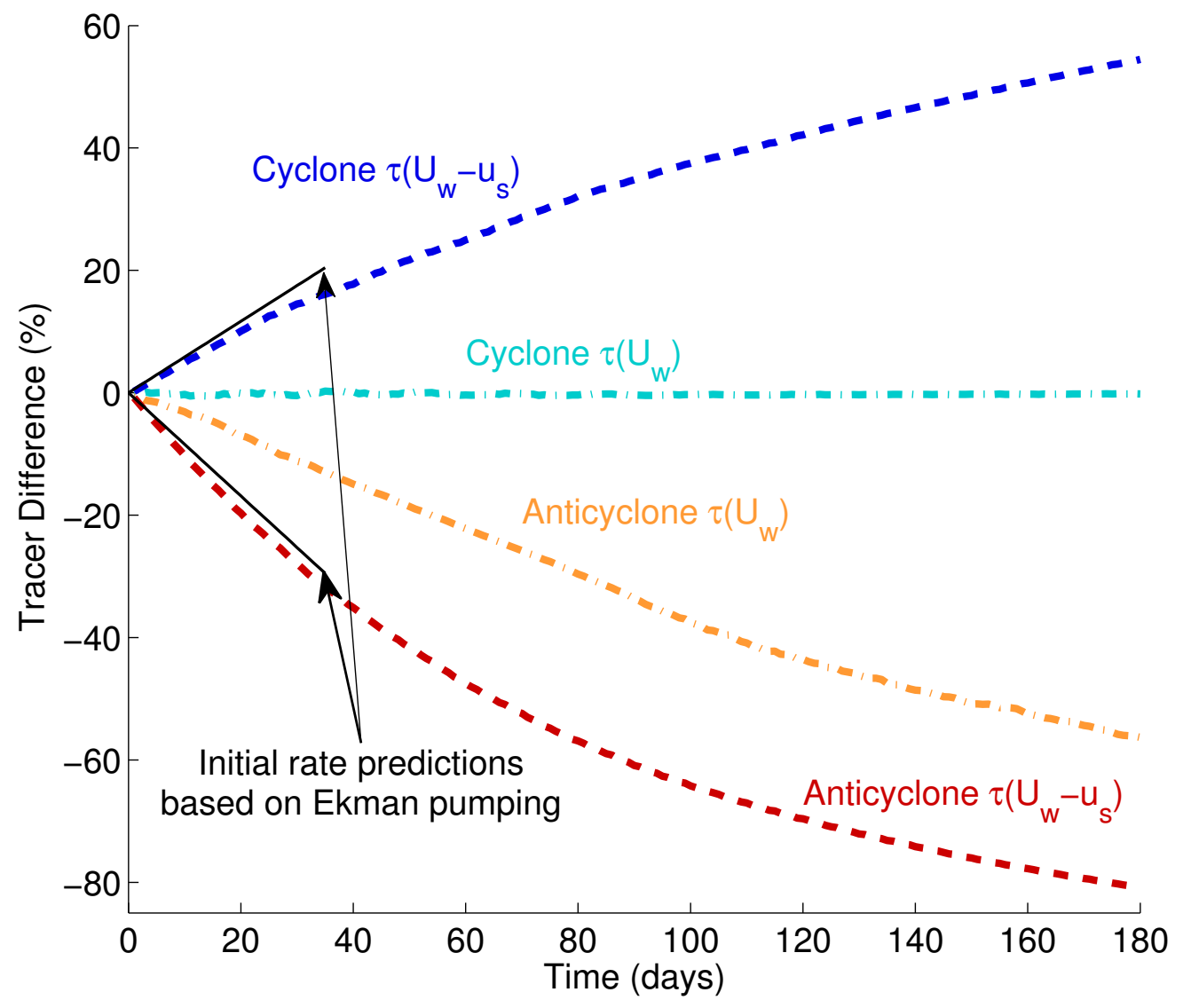

Figure 3-9: Center-edge difference in tracer concentration. Black lines represent predictions of initial aggregation/disagregation rates calculated based on Ekman pumping in idealized eddies (Equation 3.10 with eddy velocities of $v_{\theta}=0.23 \mathrm{~m} \mathrm{~s}^{-1}$ and $v_{\theta}=-0.33 \mathrm{~m} \mathrm{~s}^{-1}$, representative of model geostrophic azimuthal velocities on simulation day 1. 


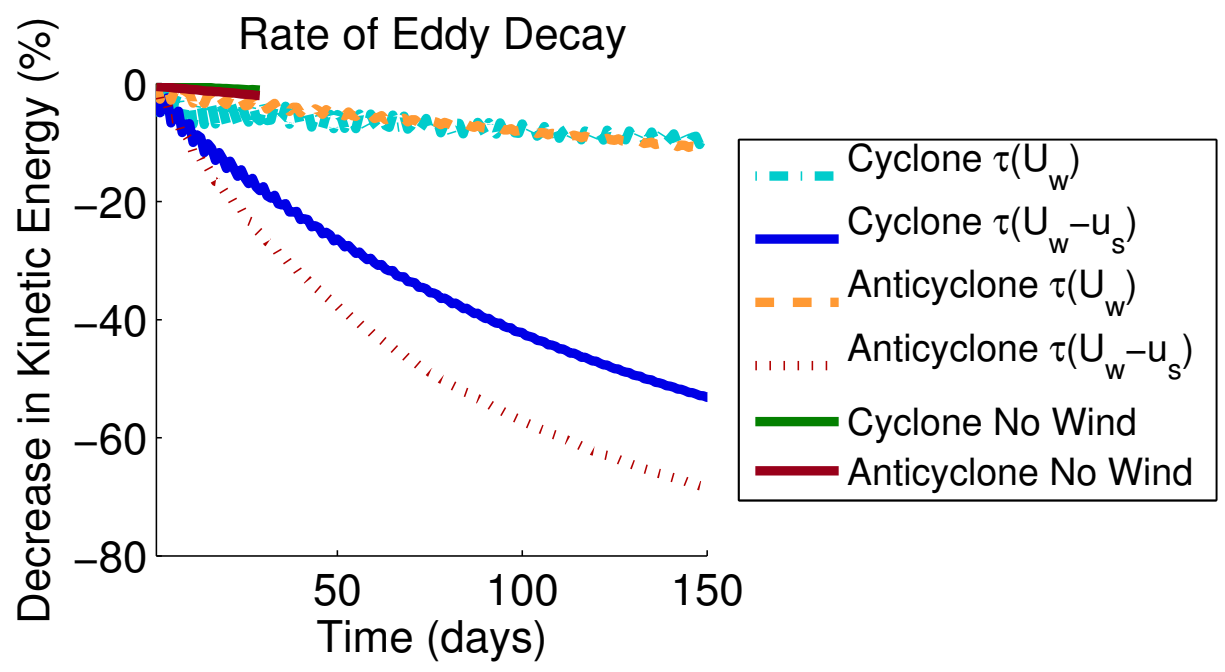

Figure 3-10: Relative decay of total kinetic energy over time.

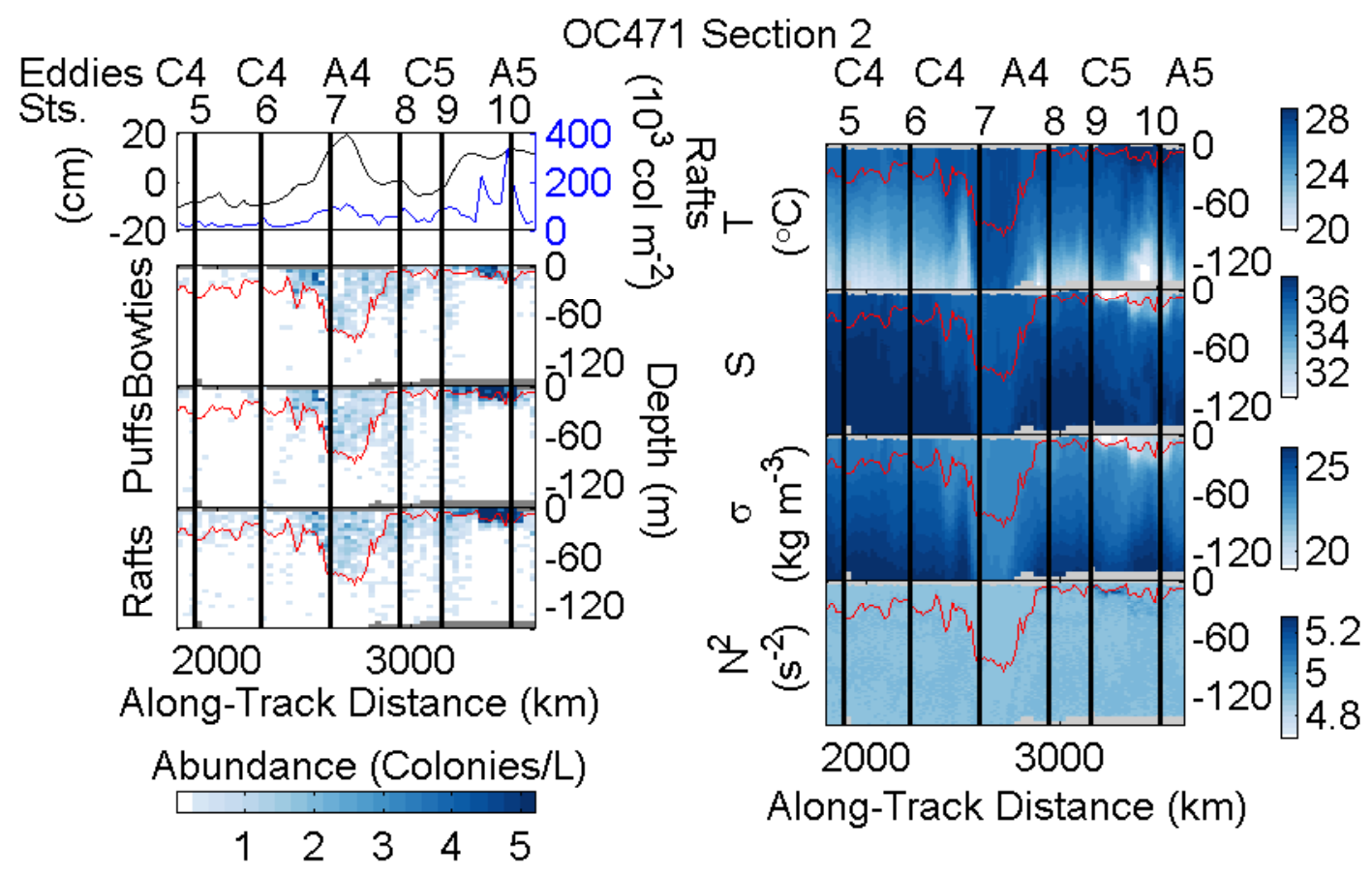

Figure 3-11: Trichodesmium abundance and physical properties associated with eddies transected on spring cruise OC471. 


\section{layer=01 salinity May 06, $201100 Z \quad[90.9 H]$}

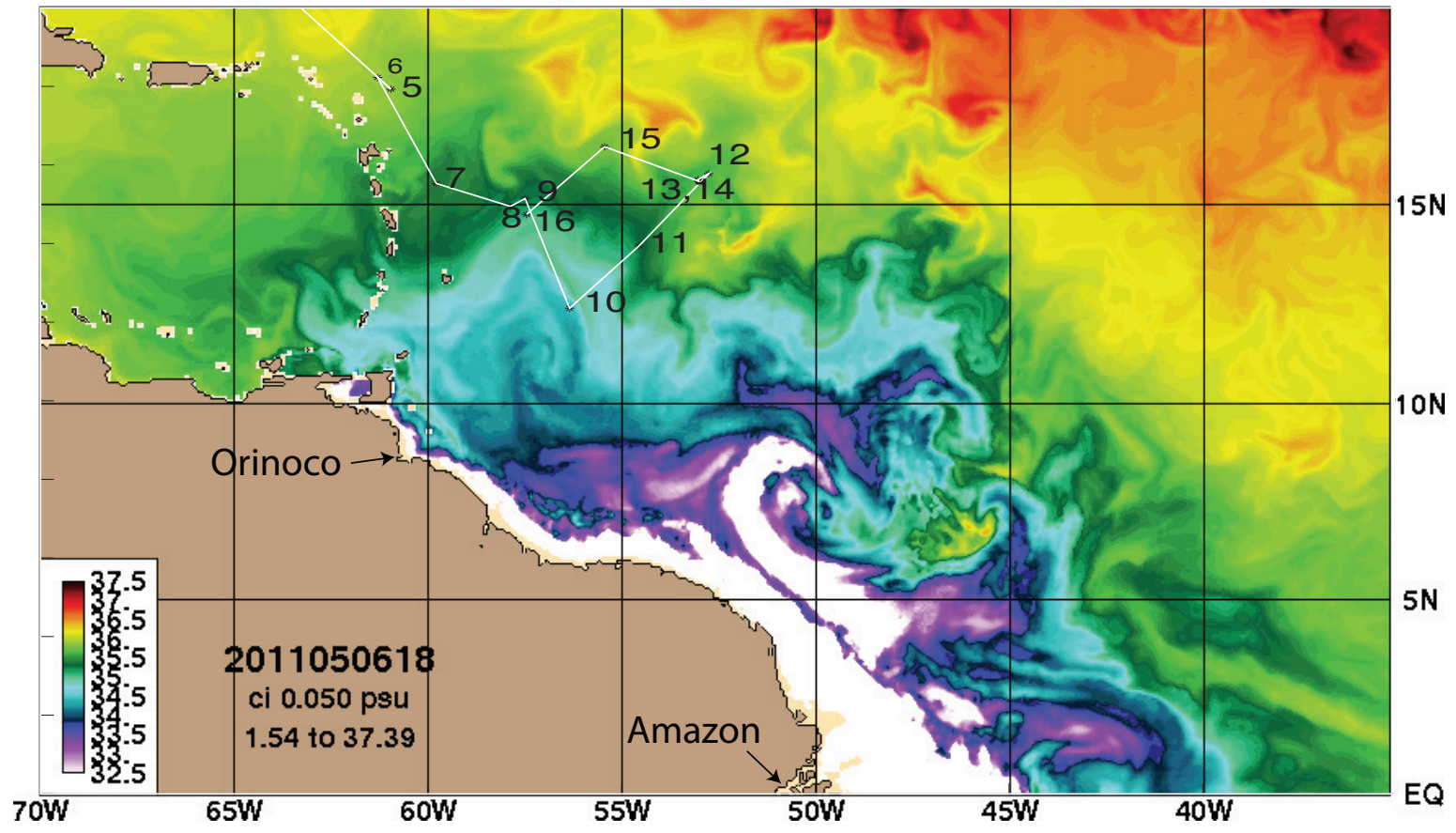

Figure 3-12: Sea Surface Salinity from NRL 1/12 ${ }^{\circ}$ Global HYCOM Hindcast (http://www7320.nrlssc.navy.mil/GLBhycom1-12/navo/nbrazlsss/nowcast/ sss2011050618_2011050600_909_nbrazlsss.001.gif). Cruise track, station locations, and Orinoco and Amazon River mouths are notated. 


\subsection{Discussion}

\subsubsection{Fall 2011: Trichodesmium Colonies in Cyclones}

Association of Trichodesmium abundance with cyclones was unexpected, as previously greater abundances were observed in anticyclones (Davis and McGillicuddy, 2006). In other instances, nutrient injection as isopycnals dome upward during eddy formation, with associated shoaling of the nutricline, has been invoked to explain elevated phytoplankton abundance in cyclones. Here, however, the pool of one of the limiting nutrients, phosphorus, is deep compared to the location of Trichodesmium colonies, found mostly within the mixed layer. Rafts, the most buoyant of Trichodesmium colony morphologies, dominated in this region. The buoyancy of Trichodesmium colonies and their surface-intensified depth distribution led to a hypothesis of physical aggregation by convergent surface currents in cyclones.

Through idealized eddy simulations based on a three-dimensional primitive equation model, we have shown that Ekman transport convergence due to the influence of eddy velocities on wind stress field can lead to patterns of buoyant tracer distribution qualitatively consistent with Fall 2010 observations. Convergence in cyclones can concentrate buoyant particles such as Trichodesmium colonies, while divergence in anticyclones can dilute them.

Quantitatively, however, simulated levels of concentration/dilution did not reach those observed in eddies C3 and A3 in Fall 2010. Assuming that the average Trichodesmium colony concentration observed over the transect spanning eddies C3 and A3 is representative of background levels, the concentration near the core of eddy C3 exhibited a 200\% increase relative to background levels, while the concentration near the core of eddy A3 exhibited an $80 \%$ decrease relative to background levels. Eddy simulations ran for 6 months, over which time buoyant tracer concentrations increased by $60 \%$ in the core of the cyclone and decreased by $80 \%$ in the core of the anticyclone. Although eddies may persist for longer than 6 months, the decrease in aggregation rate over time as well as the decay of the eddies due to the eddy-wind 

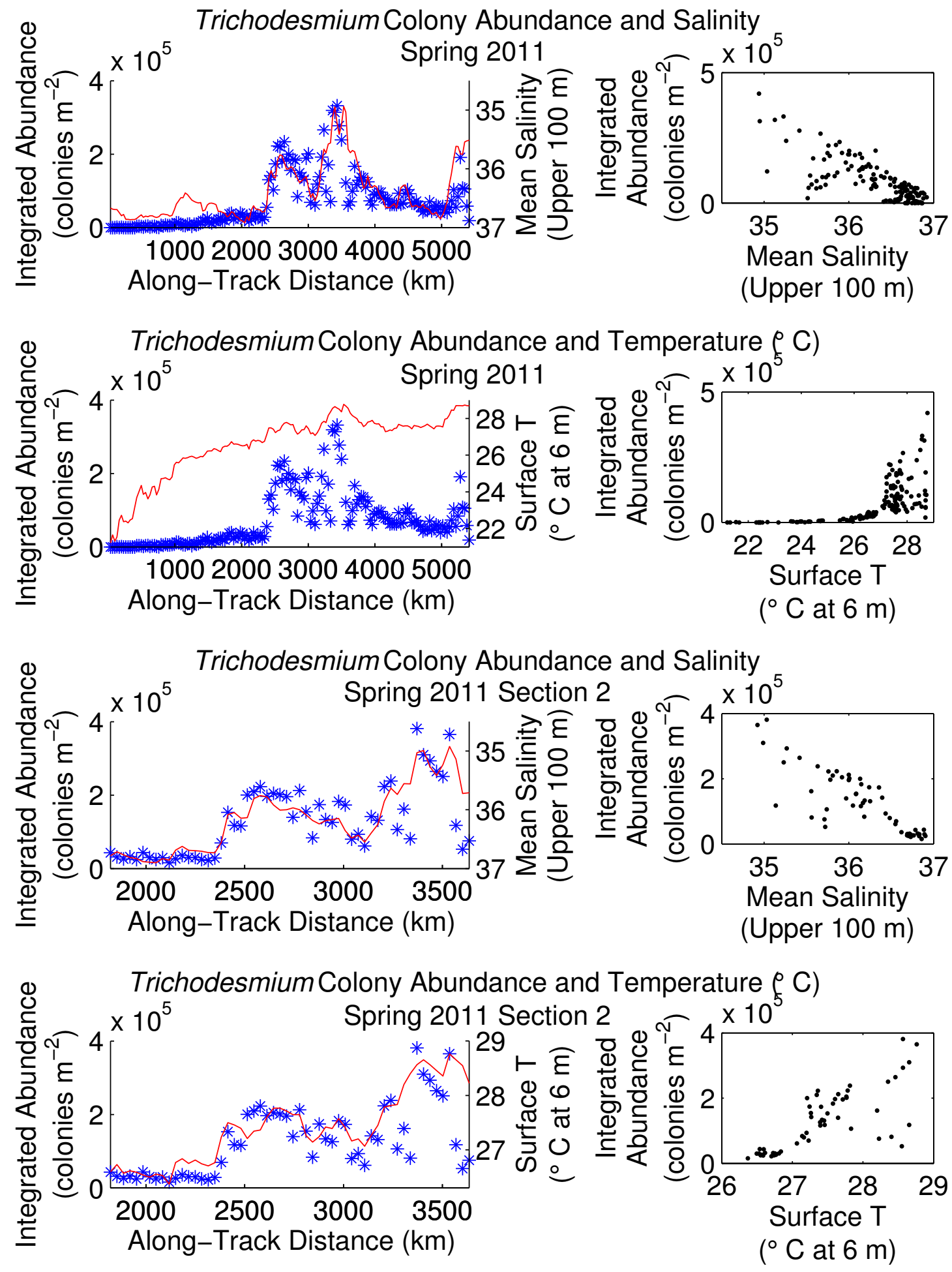
Figure 3-13: Relationship between depth-integrated Trichodesmium abundance and temperature and salinity for the entire spring cruise and for the subset of the spring cruise designated Section 2 and displayed in Figure 3-11. Red line represents salinity and blue stars represent Trichodesmium colony abundance. Salinity here is the mean over the upper $100 \mathrm{~m}$, whereas temperature the near-surface value observed at 6 $\mathrm{m}$ depth. Note that salinity axes are reversed in the first and third left panels to emphasize the degree of anticorrelation between mean salinity and depth-integrated Trichodesmium abundance.

interaction mechanism suggest that cyclone-anticyclone concentration differences as large as those observed may never be reached due to the eddy-wind interaction mechanism alone. Thus, in addition to wind-driven aggregation, spatial heterogeneity in population growth and/or mortality likely contribute to the observed patterns.

On the other hand, the estimated aggregation timescale is specific to the conditions simulated and is likely different from the actual aggregation timescale. Factors that may influence physical aggregation rates include wind speed variability and diurnal heating/cooling. Some differences between an idealized model and observations are to be expected given the simplified nature of the model, which is not intended to reproduce the full complexity of the natural environment. Additionally, we initialized the model runs with the physical characteristics of the eddy after the pattern is present, which would correspond to the end of the simulation. Based on the eddy decay in the simulation, at an earlier time the observed eddies may have had stronger sea level anomalies and azimuthal velocities. These larger velocities would be associated with greater convergence/divergence and colony accumulation/dilution. Thus, it is to be expected that the model underestimates the observed patterns in colony abundance.

Another aspect of observations not fully explained by the wind-driven aggregation model is elevated puff colony abundances in cyclones compared to anticyclones in this region. These puff colony abundances are more deeply distributed than the raft abundances. Thus, puffs may not be buoyant enough to maintain a position within the mixed layer conducive to wind-driven aggregation. One possibility is that the puff colonies may have been more buoyant at the time they were advected toward the 
center of the cyclone and since have begun to sink. Villareal and Carpenter (2003) suggest that Trichodesmium colonies may employ carbohydrate ballasting to mine phosphorus at the nutracline. Thus, if the colonies became nutrient stressed when trapped in the convergent cyclone center, the phosphorus mining mechanism could be triggered. The domed isopycnals in cyclones could facilitate this since the phosphocline might be slightly uplifted at the center of the eddy. It is unclear whether puffs and rafts would be affected by nutrient stress at different rates, but rafts are generally observed to be more buoyant and located higher in the water column (Post et al., 2002; Walsby, 1978). Another mechanism that could explain this pattern is exchange between the puff and raft morphology pools. For instance, suppose raft-shape colonies are able to transition to a puff shape or reproduction of Trichodesmium initially in raft-shaped colonies can result in some puff shaped colonies. The details of colony morphology formation are not fully understood and warrant further investigation.

Although this study shows that the physical mechanism proposed could contribute a significant portion of the variability in concentration observed between cyclones and anticyclones, it does not rule out other mechanisms. Many other factors affect Trichodesmium distributions including growth rate, which is itself influenced by environmental factors such as temperature, salinity, and nutrient availability, as well as mortality.

\subsubsection{Spring 2011: Trichodesmium Colonies in Anticyclones}

The local association of Trichodesmium colony abundance with anticyclones encountered during the spring cruise (OC471) appears at first to be consistent with the association with anticyclones observed by Davis and McGillicuddy (2006) further north in the Sargasso Sea. However, where those eddies were warm and salty, these were warm and fresh. On the spring cruise, an inverse correlation was observed between salinity and Trichodesmium colony abundance. Previous observations of Amazon plume dispersal combined with sea level anomaly observations prior to the sampling period indicate that the freshwater encountered is of riverine origin. Thus 
the elevated Trichodesmium colony abundances may be related to associated nutrient supply due to river runoff. Available phosphorus data from Spring 2010 shows some evidence of excess inorganic phosphorus emanating from the river plume. In addition, elevated Trichodesmium alkaline phosphatase activity associated with low salinity water may indicate enhanced utilization of dissolved organic phosphorus, which may be supplied through biological activity in the river plume.

River outflow has previously been indicated as a source of nutrients to Trichodesmium in the southwestern North Atlantic. Subramaniam et al. (2008) described a succession of phytoplankton functional groups downstream in a river plume. Coastal diatom species dominated until combined $\mathrm{N}$ was drawn down, followed by diatom diazotroph associations until $\mathrm{Si}$ and soluble reactive phosphorus were drawn down, with Trichodesmiumfurther downstream. That elevated Trichodesmium abundances are here found associated with riverine influence suggests that after Si is drawn down and diatoms no longer thrive, water of riverine origins may continue to supply phosphorus and possibly other nutrients necessary for Trichodesmium growth. Water emanating from river plumes was suggested as a nutrient source to Trichodesmium by Coles et al. (2004) as well. Elevated dissolved iron concentrations have been observed in the Amazon River plume (Bergquist and Boyle, 2006; Tovar-Sanchez and Sañudo-Wilhelmy, 2011) and in NBC rings containing water originating in the plume (Fitzsimmons et al., 2013). Elevated phosphorus has likewise been observed in the plume (Tovar-Sanchez and Sañudo-Wilhelmy, 2011; Sohm and Capone, 2010), particularly the SRP and particular organic phosphorus fractions. Phosphorus and iron, the two nutrients believed to be limiting to Trichodesmium growth, are thus supplied though freshwater of riverine origin. This has led to the hypothesis Trichodesmium growth fed by riverine nutrients and growth-conducive surface stratification might seed populations throughout the region. 


\subsection{Conclusions}

Two different mesoscale associations between Trichodesmium colony abundance and the physical environment were observed in the western tropical North Atlantic. Elevated Trichodesmium abundances were attributed to riverine freshwater supply associated with anticyclonic eddies, consistent with the ecological succession described by Subramaniam et al. (2008) within river plume waters. Greater colony abundances observed in cyclones were hypothesized to result from aggregation through convergent Ekman transport. The potential action of this mechanism was supported by an analytical model and idealized simulations, although additional factors must be invoked to fully explain the magnitude of the observed enhancement.

The two patterns observed are likely to prevail under very different ecological conditions. In order for the relatively slow physical aggregation mechanism to produce observable patterns, spatial variation in population growth must be small. This requirement is more likely to be met in low-growth conditions. In contrast, under bloom conditions, given Trichodesmium doubling times in the range of 2.6-5 days (Rodier and Borgne, 2010; Carpenter et al., 1993), relationships with factors influencing growth rate, particularly nutrient availability and temperature dependence, are likely to dominate. The two observed relationships with the eddy field are distinct from that observed in the subtropical North Atlantic (Davis and McGillicuddy, 2006), which has recently been attributed to a combination of down-gradient transport of biomass and phosphorus by anticyclones originating in the Gulf Stream (McGillicuddy, 2014). These findings underscore the importance of physical processes in shaping abundance patterns underlying important biogeochemical processes such as carbon and nitrogen cycling in the world's tropical oceans.

We conclude that wind-driven aggregation could produce the observed pattern of association of Trichodesmium abundance with cyclones observed on the fall cruise (OC469), and that freshwater input was the driving factor controlling the apparent association with anticyclones on the spring cruise (OC471). Future work including 
analysis of wind-driven aggregation through more sophisticated and higher resolution models could further elucidate the relationship between Trichodesmium colonies and their physical environment. A model of two interacting eddies together could be used to determine whether buoyant tracer originating in the anticyclone transferred to the cyclone and on what timescales. Patterns of buoyant tracer imbedded in basinscale eddy-resolving model with a realistic wind field could provide assessment of the mechanism in a more realistic simulation. 

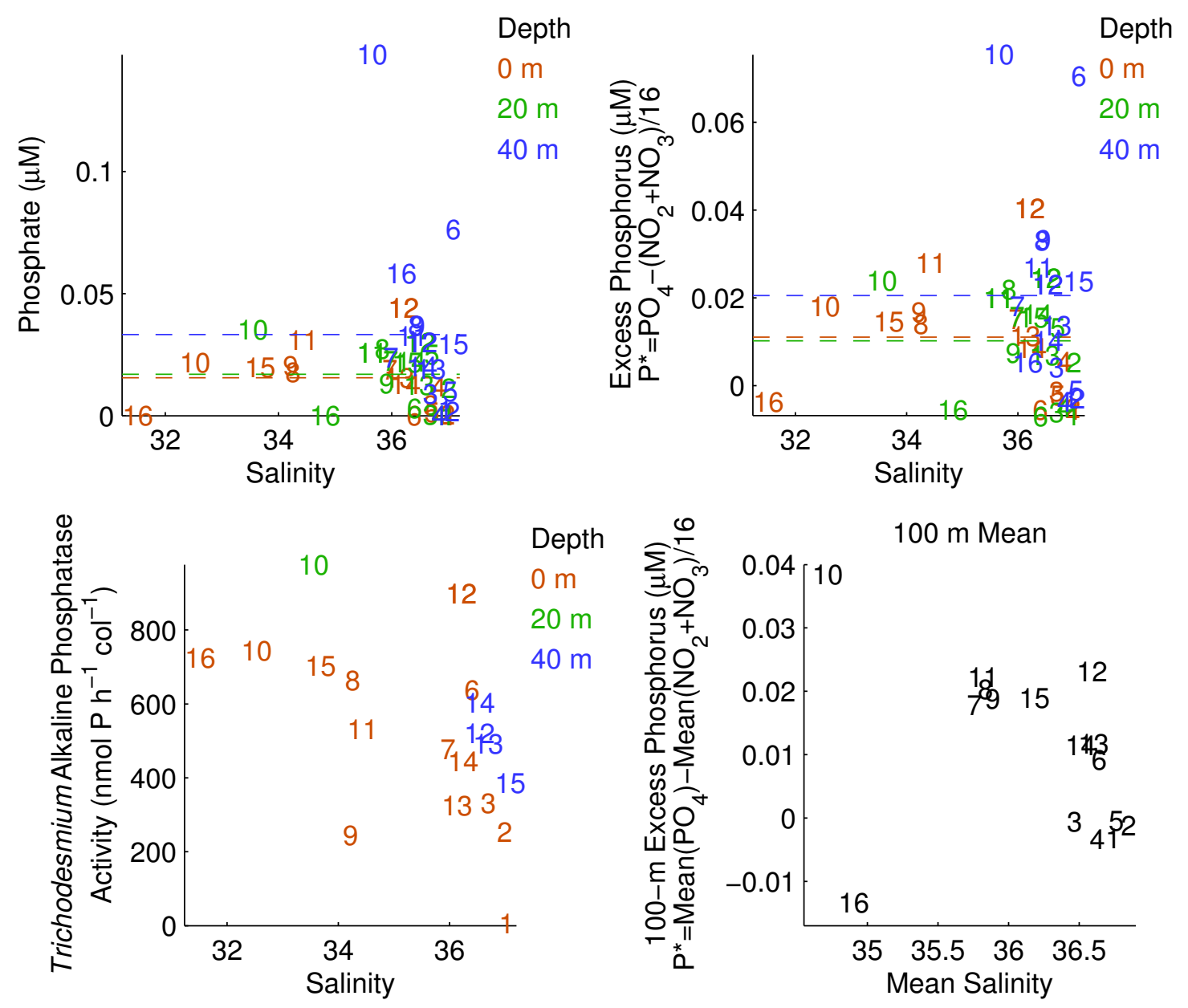

Figure 3-14: Relationship between phosphorus and salinity. Numbers shown identify stations; color indicates sample depth. Upper left: Phosphate vs. salinity. Upper right: Excess phosphate vs. salinity. Lower left: Trichodesmium alkaline phosphatase activity vs. salinity. Samples matched to $0 \mathrm{~m}$ salinity were actually taken from net tows at $5 \mathrm{~m}$ depth. Lower right: Excess phosphate based on mean phosphate and nitrite+nitrate concentrations over the upper $100 \mathrm{~m}$ vs mean salinity in the upper 100 $\mathrm{m}$. Measurement detection and quantification limits for phosphate and nitrite+nitrate are: $\mathrm{PO}_{4} \mathrm{DL}=0.0025 \mu \mathrm{M}, \mathrm{QL}=0.08 \mu \mathrm{M} ; \mathrm{NO}_{2}+\mathrm{NO}_{3} \mathrm{DL}=0.05 \mu \mathrm{M}, \mathrm{QL}=0.18 \mu \mathrm{M}$. 
Chapter 4

How much do deep populations

contribute to nitrogen fixation by

Trichodesmium spp. in the

tropical-subtropical North

Atlantic? 


\subsection{Abstract}

This study evaluates the hypothesis of Davis and McGillicuddy (2006) that Trichodesmium nitrogen fixation in the North Atlantic may be underestimated by conventional sampling methods, based on their finding of higher than expected colony abundances at depth using the VPR in the subtropical North Atlantic. Here, Trichodesmium colony abundances are estimated from VPR transects completed on two cruises in the tropical and subtropical North Atlantic in fall 2010 and spring 2011. A bio-optical model is developed based on carbon-normalized nitrogen fixation rates measured on those cruises. The fit is based on the relationship between nitrogen fixation and incubation irradiance. PAR is estimated from SeaWiFS climatologies and vertical attenuation coefficients, which are estimated from in situ measurements. Estimates of abundance and nitrogen fixation are similar in magnitude and vertical and geographical distribution to estimates compiled in a database by Luo et al. (2012). Thus, in the mean, VPR-based estimates of volume-specific nitrogen fixation rates at depth in the tropical North Atlantic are not inconsistent with estimates derived from conventional sampling methods. Based on this analysis, if Trichodesmium nitrogen fixation is underestimated, it is unlikely that it is due to underestimation of deep abundances due to mechanical disturbance during net-based sampling.

\section{$4.2 \quad$ Introduction}

The quantification of oceanic nitrogen fluxes has been approached in several ways. Nitrogen fixation rates have been measured directly through incubation experiments, but this is a short-term measurement, integrating over timescales of hours or days. Many such measurements taken together may on average give a reasonable estimate of longer-term nitrogen fixation rates, but unresolved variability could lead to skewed estimates. Additionally, the potential exists for error due to the necessary exposure of the nitrogen fixing organisms to unnatural conditions during incubation. At the other end of the spectrum, geochemical analysis looks at markers of nitrogen fixation, 
primary production, and nitrate flux that reflect larger spatial and temporal scales. For instance, excess nitrate $(N *)$ combined with information about the ventilation of various water masses has been used to estimate nitrogen fixation (Gruber, 2008). Yet another approach is to measure rates of denitrification and assume that the system is in steady state over some timescale, and therefore that sources and sinks must be equal. However, this approach has large uncertainty due to potentially unresolved spatial and temporal variability as well as uncertainty in the steady state assumption. Comparisons have shown direct measurements of nitrogen fixation from incubation experiments to be low compared to geochemical estimates by a factor of one quarter to one half (Mahaffey et al., 2005).

The differences could be due to a variety of factors. Each estimate could be correct over its relevant timescale if nitrogen fixation rates vary over time. Nitrogen fixation could be under-sampled in time and space by labor-intensive incubation experiments, particularly if a significant component of nitrogen fixation is due to transient phytoplankton blooms or localized regions which may have escaped sampling. The deficit could be made up through nitrogen fixation by previously overlooked groups such as symbiotic cyanobacteria or heterotrophic bacteria (Mahaffey et al., 2005).

A study by Davis and McGillicuddy (2006) suggested yet another possibility. Their video plankton recorder (VPR) survey captured greater than expected Trichodesmium colony abundance at depth in the northern limb of the subtropical gyre in the North Atlantic. A further VPR study in the Caribbean (Davis et al., 2013) encountered uniform Trichodesmium distribution with depth within the upper $120 \mathrm{~m}$ over part of the survey region. These differences from other studies could be due to the VPR's noninvasive sampling technique. In contrast, net tows may disrupt some colonies, particularly those sampled at depth that must travel farther in the net to reach the surface. Davis and McGillicuddy (2006) posited that if similarlyshaped abundance profiles were present in the regions where Trichodesmium was most abundant, such as the southwestern North Atlantic, deep Trichodesmium populations could account for the "missing" nitrogen fixation as estimated from incubation exper- 
iments and abundance data. The question remained open as the transatlantic study was outside the range where Trichodesmium is most abundant, and the Caribbean study focused on a limited geographical range without measurement of nitrogen fixation rates. Here, we evaluate that hypothesis using data from fall 2010 and spring 2011 cruises in the Western subtropical-tropical Atlantic. Two bio-optical nitrogen fixation models, developed from nitrogen fixation assays carried out on those cruises, are used to infer nitrogen fixation rates from VPR abundance data. The data additionally provide the opportunity to examine mesoscale variations in nitrogen fixation rates and their association with physical processes.

\subsection{Methods}

\subsubsection{Data Collection}

Sampling occurred on two cruises on the R/V Oceanus, OC469 from October 122, 2010, and OC471 from April 23-May 13, 2011. The planned cruise track consisted of a meridional section from Bermuda to Puerto Rico, followed by a southeastward leg to a longitude of approximately $40^{\circ} \mathrm{W}$ and a southwestward leg ending just east of Barbados. Precise sampling locations (Figures 4-1 and 4-2; Table 4.1) were chosen during the cruises as informed by satellite observations of sea surface height, real-time analysis of VPR Trichodesmium abundance observations, and ocean-color-based estimates of Trichodesmium bloom probability (Westberry and Siegel, 2006; Westberry et al., 2005) provided by Toby Westberry. This adaptive strategy allowed sampling to be directed toward interesting physical and biological features such as eddies and areas of elevated Trichodesmium abundance. On OC469 the ship was diverted westward early on due to an approaching tropical cyclone, but soon resumed its intended course. A series of cyclonic and anticyclonic eddy features were transected during each cruise. 


\begin{tabular}{lllllllll}
\hline & \multicolumn{3}{l}{ OC469 } & & & \multicolumn{3}{l}{ OC471 } \\
St. & Lat. & Lon. & Date & & St. & Lat. & Lon. & Date \\
\cline { 1 - 4 } 2 & 31.687 & 64.105 & 05 Oct. 2010 & & 1 & 31.668 & 64.166 & 26 Apr. 2011 \\
3 & 28.587 & 65.058 & 06 Oct. 2010 & & 2 & 25.500 & 66.233 & 28 Apr. 2011 \\
4 & 28.969 & 65.988 & 07 Oct. 2010 & & 3 & 22.181 & 65.939 & 29 Apr. 2011 \\
5 & 29.082 & 66.109 & 08 Oct. 2010 & & 4 & 20.010 & 63.290 & 30 Apr. 2011 \\
6 & 25.512 & 67.253 & 10 Oct. 2010 & & 5 & 17.928 & 60.953 & 01 May 2011 \\
7 & 22.491 & 67.303 & 11 Oct. 2010 & & 6 & 18.226 & 61.297 & 02 May 2011 \\
8 & 21.266 & 64.003 & 12 Oct. 2010 & & 7 & 15.529 & 59.783 & 03 May 2011 \\
9 & 17.254 & 58.505 & 14 Oct. 2010 & & 8 & 14.945 & 57.869 & 04 May 2011 \\
10 & 15.227 & 54.659 & 15 Oct. 2010 & & 9 & 15.146 & 57.492 & 05 May 2011 \\
11 & 13.491 & 51.309 & 16 Oct. 2010 & & 10 & 12.353 & 56.341 & 06 May 2011 \\
12 & 11.915 & 48.459 & 17 Oct. 2010 & & 11 & 13.940 & 54.550 & 07 May 2011 \\
13 & 11.537 & 51.357 & 18 Oct. 2010 & & 12 & 15.777 & 52.754 & 08 May 2011 \\
14 & 12.209 & 54.520 & 19 Oct. 2010 & & 13 & 15.626 & 53.032 & 09 May 2011 \\
15 & 12.896 & 58.594 & 20 Oct. 2010 & & 14 & 15.587 & 53.010 & 10 May 2011 \\
15.1 & 12.563 & 56.663 & 21 Oct. 2010 & & 15 & 16.463 & 55.440 & 11 May 2011 \\
16 & 12.547 & 56.340 & 21 Oct. 2010 & & 16 & 14.741 & 57.455 & 12 May 2011 \\
17 & 12.565 & 56.622 & 21 Oct. 2010 & & & & \\
\hline
\end{tabular}

Table 4.1: Station locations and dates for both cruises. Latitudes are in degrees North and longitudes in degrees West. 


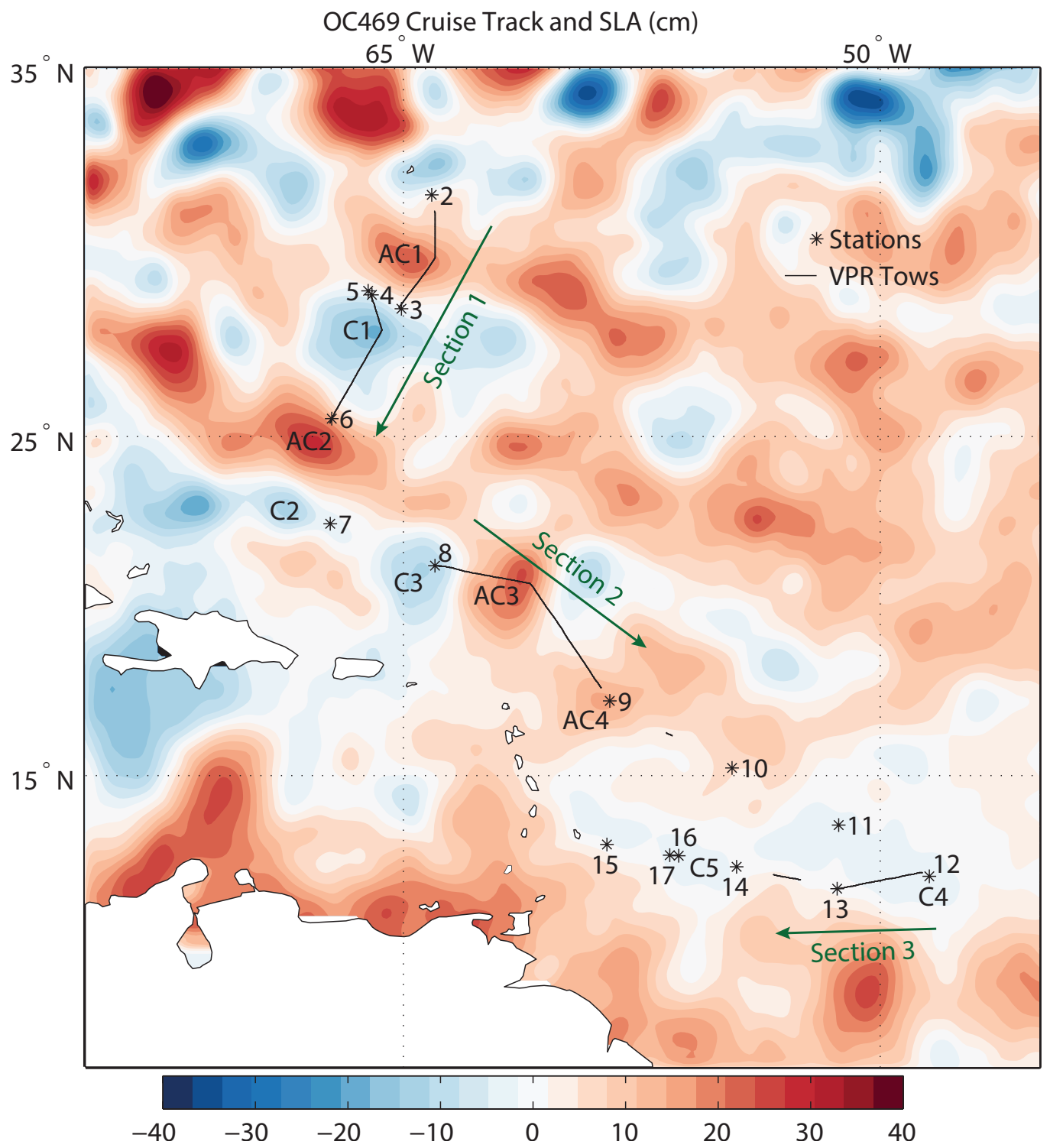

Figure 4-1: Locations of stations and VPR tows for cruise OC469 with sea level anomaly. Black lines indicate VPR tows. Stars indicate station locations. Targeted cyclones and anticyclones are labeled with prefixes "C" and "AC", respectively. Labeled sections are referred to in subsequent analysis. 


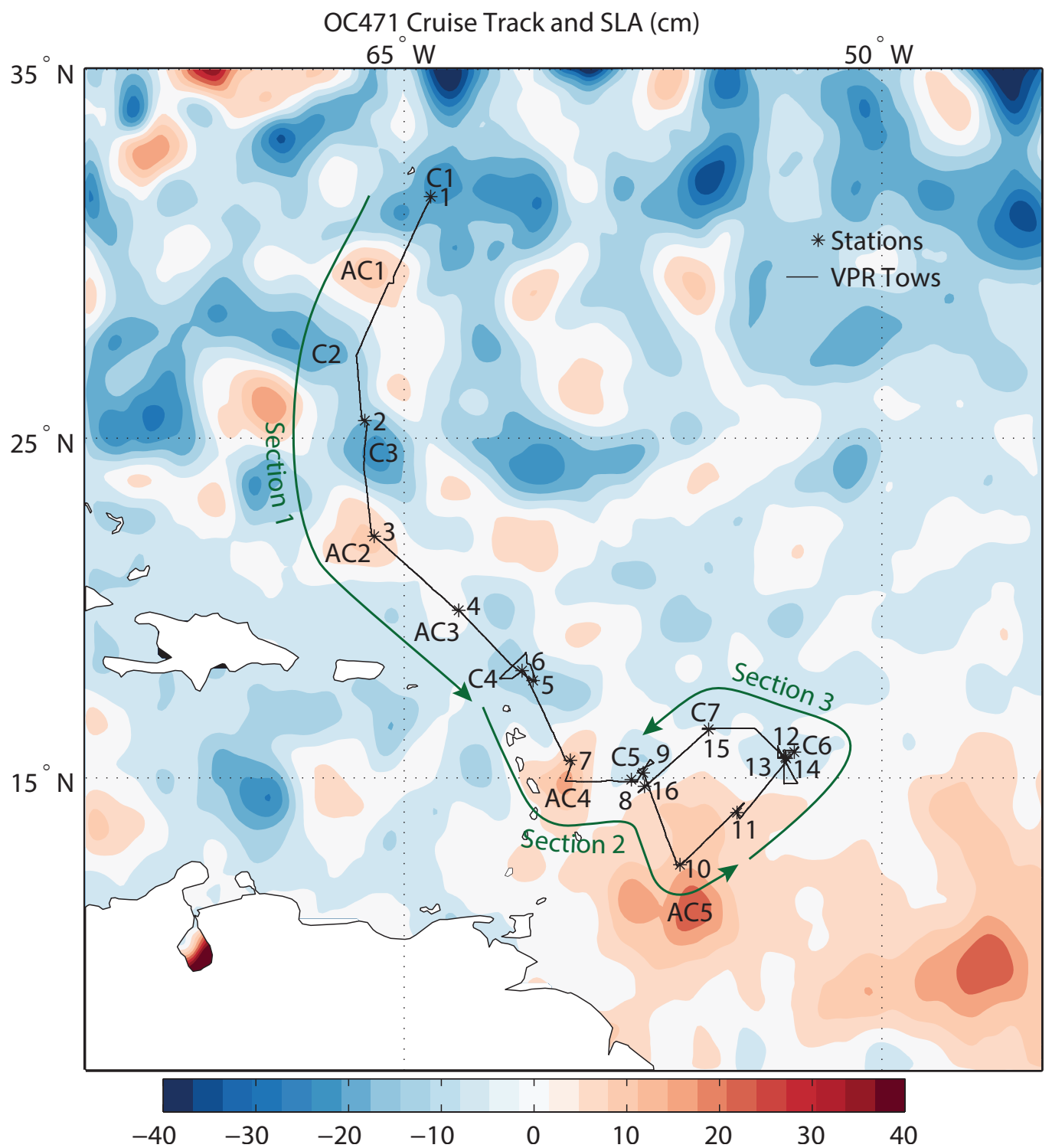

Figure 4-2: Locations of stations and VPR tows for cruise OC471 with sea level anomaly. Black lines indicate VPR tows. Stars indicate station locations. Targeted cyclones and anticyclones are labeled with prefixes "C" and "AC", respectively. Labeled sections are referred to in subsequent analysis. 


\section{VPR Sampling}

Trichodesmium colony abundance was sampled by the VPR, towed between stations (Figures 4-1 and 4-2). The VPR consists of a towed body, containing a CTD and a synchronized video camera and xenon strobe (Davis et al., 2005). Data are sent in real-time through a fiber-optic cable to a shipboard computer system. The VPR undulates between the surface (5-10 m) and approximately $120 \mathrm{~m}$ depth, completing an up-down cycle approximately every six minutes. Since it is towed at 10 knots (5.1 $\mathrm{m} / \mathrm{s}$ ), this corresponds to one vertical cycle every $1.8 \mathrm{~km}$. Navigation to starboard of the ship's wake near the surface, in addition to strategic camera and strobe placement and towfish design, minimize disturbance of the regions sampled. At a frame rate of 30 $\mathrm{Hz}$, each 984 x 1009 pixel video frame is passed through object-identification software to pick out "regions of interest" and save them, using a time-stamp naming convention, to a hard disk. In addition to imaging, the VPR recorded pressure, temperature, salinity, photosynthetically active radiation (PAR), oxygen, and fluorescence.

Images were initially sorted using image recognition software (Hu and Davis, 2006). For OC471, due to the large number of images collected, the sample size was reduced to 1000 images per sampling hour, evenly spaced among the collected images (Chapter 2). Abundances were re-scaled accordingly. For both cruises, each classified image was manually checked and reclassified as necessary, into the three Trichodesmium morphological categories, puff, raft, and bowtie, and an "other" category containing everything else. Trichodesmium puff, raft, and bowtie colonies are readily sampled using the VPR system due to their macroscopic size, but individual trichomes are not distinguishable from other similarly shaped bodies.

The volume of the field of view of was calibrated before each cruise by the tethered copepod method described by Davis et al. (2005) and was $6.929 \mathrm{~mL}$ and $15.168 \mathrm{~mL}$ for OC469 and OC471, respectively. These volumes are used to calculate abundances from observation frequencies. The corresponding vertical and horizontal dimensions of the field of view were $13 \mathrm{~mm} \times 13 \mathrm{~mm}$ and $12 \mathrm{~mm}$ x $12 \mathrm{~mm}$; the camera and strobe were oriented to produce a deeper and narrower field of view for OC471. Abundance 
data were gridded to bins $5 \mathrm{~m}$ deep and 11 and $33 \mathrm{~km}$ wide for OC469 and OC471, respectively. Within each bin, abundances were estimated under the assumption of a Poisson distribution, based on the total water volume imaged and the number of positive identifications.

Physical and bio-optical data from the VPR were binned to the same horizontal resolution as the abundance data and either 1 or $5 \mathrm{~m}$ vertically, as indicated. Mixed layer depths were diagnosed using a threshold criterion of $0.025 \mathrm{~kg} \mathrm{~m}^{-3}$ difference in potential density from the surface, based on binned data at $1 \mathrm{~m}$ vertical resolution. Advantages of using the VPR include the noninvasive nature of its sampling method and high spatial resolution facilitating observation of phenomena occurring at the mesoscale and even sub-mesoscale.

\section{Nitrogen Fixation Rate Measurements}

The sampling program included daily stations with associated nitrogen fixation experiments beginning at approximately 10:00 a.m. local time. Trichodesmium colonies for on-board incubation experiments and genetic assays were picked individually with pipettes from water collected at the surface (5-15 m) and at depth (20-70 m). Surface and deep samples were collected by pumping water into a $130 \mu \mathrm{m}$ net on OC469 and by MOCNESS with $150 \mu \mathrm{m}$ nets on OC471. Additional near-surface samples were taken by manual $1 / 3 \mathrm{~m}$ hoop net tows $(130 \mu \mathrm{m})$ between the surface and approximately 10 $\mathrm{m}$ depth on both cruises. After initial collection, the largest and most intact individual colonies were isolated using eyedroppers and transferred to filtered seawater for incubation experiments in order to assemble sufficient biomass to produce measurable rates. Nitrogen fixation was measured by acetylene reduction assay (Capone and Montoya, 2001) with a 3:1 acetylene reduction to nitrogen fixation ratio (Heithoff, 2011). Incubations lasted approximately four hours at temperatures of $26.0-27.5^{\circ} \mathrm{C}$ and irradiances generally corresponding to in situ PAR within the limitations of the incubators. The maximum irradiances attainable were $400 \mu \mathrm{mol}$ quanta $\mathrm{m}^{-2} \mathrm{~s}^{-1}$ for OC469 and $700 \mu \mathrm{mol}$ quanta $\mathrm{m}^{-2} \mathrm{~s}^{-1}$ for OC471. Additional deck-board nitrogen fix- 
ation experiments were carried out on OC471 at natural irradiances ranging from 1000-1600 $\mu$ mol quanta $\mathrm{m}^{-2} \mathrm{~s}^{-1}$.

For each nitrogen fixation sample, the number of puff and raft colonies was recorded. Colonies were filtered onto a combusted GF/F filter using vacuum filtration, and CHN analysis was performed by the Marine Biological Laboratory Stable Isotope Laboratory, Woods Hole, MA. Mean carbon content per puff and raft colony was estimated by regressing carbon content with number of puffs and number of rafts for each cruise. This calculation was based on 101 samples for OC469 and 97 samples for OC471. Bowtie carbon content per colony was assumed the same as puff carbon per colony.

\section{Hydrography and Discrete Water Sampling}

At each station, CTD casts measured temperature, salinity and PAR. Water samples were collected with a rosette system in 10 L Niskin bottles at depths of 700, 500, $300,200,100,80,60,40,20 \mathrm{~m}$, and the surface. Water for nutrient analyses was 0.2 $\mu \mathrm{m}$ filtered into acid-cleaned bottles and stored frozen. In the upper $80 \mathrm{~m}$, additional water samples were taken for the microscopic enumeration of Trichodesmium colonies and free trichomes by methods described by Carpenter et al. (2004). In brief, the samples were gravity filtered onto $10 \mu \mathrm{m} 47 \mathrm{~mm}$ filters through in-line filter holders attached to the spigot of the Niskin bottles. After filtration the filters were fixed for 30 minutes in the filter holders with $4 \%$ paraformaldehyde in $0.2 \mu \mathrm{m}$ filtered sea water. Paraformaldehyde was removed and the filters were then mounted in immersion oil on slides with coverslips. The fixed preparations were stored frozen prior to enumeration. Trichodesmium spp. colonies and filaments (trichomes) were identified and counted with epifluorescence microscopy of phycoerythrin autofluorescence by imaging the entire filter. Colonies were defined as associations of 8 or more filaments. Colonies and filaments were enumerated in 70 samples from 14 stations from OC469. From OC471, colonies were enumerated in 43 samples from 9 stations, and filaments were enumerated in 39 samples from the same 9 stations. 
Microscopic colony counts were compared to VPR colony abundances to confirm the calibration of the VPR sampling volume and the image subsampling method applied to OC471 (see Chapter 2).

\section{Sea Level Anomaly}

Sea level anomalies presented here were calculated by objective analysis of available altimetry data.

\subsubsection{Data Analysis and Modeling}

\section{Bio-optical Models of Nitrogen Fixation}

Several studies have developed a functional relationship between nitrogen fixation, $N F$, and irradiance, $I$, based on a saturating response of nitrogen fixation to PAR. Hood et al. (2002) based such a relationship on Platt et al. (1980)'s parameterization of photosynthesis as a function of irradiance including photoinhibition. The fit was based on observations from the tropical Atlantic, where samples were collected from a range of depths and incubated at corresponding irradiances (Equation 4.1).

$$
N F=\mu_{1}\left(1-\exp \left(-\frac{I}{I_{s}}\right)\right) \exp \left(-\frac{I}{I_{b}}\right)
$$

In the absence of photoinhibition, the relationship simplifies to Equation 4.2.

$$
N F=\mu_{\max }\left(1-\exp \left(-\frac{\alpha I}{\mu_{\max }}\right)\right)
$$

Breitbarth et al. (2008) fit chl- $a$-normalized nitrogen fixation to an equation of the form used by Jassby and Platt (1975) to model the photosynthesis-irradiance relationship,

$$
N F=\mu_{\max } \tanh \left(\frac{\alpha I}{\mu_{\max }}\right)
$$


The relationship has also been modeled using a Michaelis-Menten-type equation (Davis and McGillicuddy, 2006):

$$
N F=\mu_{\max } \frac{I_{F}}{\frac{\mu_{\max }}{\alpha}+I_{F}} .
$$

In the Davis and McGillicuddy (2006) model, $I_{F}$ is the percent of surface irradiance at depth. Hourly rates are converted to daily rates under the assumption that the daily nitrogen fixation curve can be represented by a half sinusoid spread over twelve hours, taking into consideration the incubation duration.

Equations 4.2, 4.3, and 4.4 have the common characteristics of constant slope $\alpha$ in the limit as irradiance approaches zero and an upper limit of $\mu_{\max }$ for large irradiance. They vary in the transition from linear slope to the saturating value; for a given $\alpha$ and $\mu_{\max }$, the transition is most abrupt in Equation 4.3. When the parameters are fit to incubation irradiance and POC-normalized nitrogen fixation rates from shipboard incubations of field-collected colonies from OC469 and OC471, the resulting curves are nearly identical within the range of observed irradiances. Therefore, choice of the hyperbolic tangent function of Jassby and Platt (1975) (Equation 4.3) for the current study was somewhat arbitrary.

The parameters associated with the relationship were fit by maximum likelihood estimation under an assumption of normally distributed random error. Ninety-five percent confidence intervals for each parameter were estimated by profile log likelihood with all other parameters held at maximum likelihood values. Available data from both cruises were used to fit the model. Surface-collected, deep, and hoop net-collected samples all contribute to the fit.

For comparison, we also present results from the model used by Davis and McGillicuddy (2006). However, they framed their model in terms of per-colony nitrogen fixation with a surface rate of $0.7 \mathrm{nmol} \mathrm{N}(\mathrm{mol} \mathrm{C})^{-1} \mathrm{~h}^{-1}$ based on nitrogen-fixation incubations on diver-collected colonies reported by Orcutt et al. (2001). Rates from diver-collected colonies were approximately four times higher than corresponding rates measured on net-sampled colonies Orcutt et al. (2001). In 
order to carry out a more balanced comparison between the Davis and McGillicuddy (2006) model and the new model, we have scaled the surface abundance to reflect the overall mean per-colony surface $(\leq 10 \mathrm{~m})$ abundance from OC469 and OC471 of 0.13 $\mathrm{nmol} \mathrm{N}(\mathrm{mol} \mathrm{C})^{-1} \mathrm{~h}^{-1}$ by multiplying by a factor of $0.164(0.13 / 0.7 \times 0.6667 / 0.7353)$, equal to the ratio of nitrogen fixation rates times a time conversion factor. This time conversion factor accounts for the difference between four- and seven-hour incubations when converting hourly to daily rates under the assumption of sinusoidal modulation of the maximum rate.

\section{PAR Model}

PAR was modeled by extrapolating SeaWiFS 8-day climatological daily surface PAR to depths using attenuation coefficients estimated from VPR PAR data. Climatological SeaWiFS observations have the advantages of broader spatial and temporal coverage than the available VPR PAR measurements. The climatological values represent PAR just below the sea surface under average conditions, including the effects of cloud-cover. Eight-day periods close to the middle of each cruise were selected from available SeaWiFS climatologies. The period chosen for OC469 was Oct 8-15 (1997-2010); May 1-8 (1998-2010) was chosen for OC471.

The physics of light transmission through water are complex as both absorption and scattering contribute to attenuation. In the idealized case of a thin parallel beam, the radiant flux diminishes exponentially along its path according to Equation 4.5 with the beam attenuation coefficient, $c$, equal to the sum of the absorption and scattering coefficients.

$$
\phi=\phi_{0} e^{-c r},
$$

where $r$ is the path length (Kirk, 1994). However, in the real ocean, photons may be scattered one or more times before being absorbed. By changing its direction, scattering increases a photon's path length through the water, leading to greater 
probability of absorption. Scattering can also add to the light in a given beam if photons from a nearby beam are scattered into it. The proportion of diffuse (already scattered) light will tend to increase with depth as more and more scattering events take place. Because scattering increases path length, and a higher proportion of diffuse light is present at depth, light can attenuate more quickly at depth. On a cloudy day, however, much of the light that reaches the surface of the ocean is already diffuse.

Notwithstanding these and other complications, it is often possible to determine a vertical attenuation coefficient that is approximately constant in time for a given water composition, regardless of time of day (Kirk, 1994). The vertical attenuation coefficient, $k$, is defined as in Equation 4.6.

$$
k=-\frac{d \ln E_{d}}{d z}
$$

This vertical attenuation coefficient for downward flux can be applied with reasonable accuracy to scalar irradiance as well (Kirk, 1994). As Kirk (1994, p. 152) points out, scalar irradiance, the total light incident on a point from all angles, is more relevant than downwelling irradiance in determining light availability to planktonic organisms. The difference in attenuation coefficient for downward versus scalar irradiance is small for clear oceanic waters such as were observed throughout most of OC469 and OC471 (factor of up to 1.2), but in the range of 1.4-2.5 for turbid coastal waters.

Thus, attenuation coefficients were estimated from VPR PAR data binned to 5-m vertical resolution using Equation 4.6 with the derivative estimated by central difference. Quality control was achieved by eliminating bins with less than 545 or more than 2364 data points per bin and PAR values greater than $1150 \mu$ mol quanta $\mathrm{m}^{-2} \mathrm{~s}^{-1}$ or less than $10 \mu \mathrm{mol}$ quanta $\mathrm{m}^{-2} \mathrm{~s}^{-1}$, to minimize the effects of noise. Attenuation coefficient estimates clustered around a single value for OC469 and two values, one with salinities greater than 35.4 and one with salinities less than 35.4, for OC471. Therefore, a single value for OC469 and dual values for OC471 were chosen to minimize the least squares fit between the VPR-observed and estimated PAR fields. The 
vertical attenuation coefficient was assumed constant from the uppermost available value to the surface.

Hourly surface PAR input to the instantaneous PAR nitrogen fixation model was estimated from daily PAR using Equation 4.7.

$$
I / I_{d}=\frac{\pi}{24}\left(A_{1}+B_{1} \cos (W 2)\right) \frac{\cos (W 2)-\cos (W 1)}{\sin (W 1)-W 1 \cos (W 1)}
$$

where

$$
A_{1}=0.409+0.5016 \sin \left(W 1-\frac{\pi}{3}\right)
$$

and

$$
B_{1}=0.6609-0.4767 \sin \left(W 1-\frac{\pi}{3}\right),
$$

and all angles are in radians (Collares-Pereira and Rabl, 1979; Brock, 1981).

$$
W 1=\arccos (-\tanh (L) \tanh (D 1))
$$

is the sunset hour-angle, with $L$ the latitude and $D 1$ the declination,

$$
D 1=23.45 \frac{\pi}{180} \sin \left(\frac{360(284+N)}{365} \frac{\pi}{180}\right)
$$

with $\mathrm{N}$ the Julian Day. $W 2$ is the hour-angle at the given time of day,

$$
W 2=(t-12) \times 15
$$

if $t$ is the time of day in hours past midnight. PAR estimates were extended to depths associated with binned abundances by integrating Equation 4.6 over each bin using the attenuation coefficients estimated from the available VPR data. Climatological PAR estimates were compared to PAR measured by the more recently calibrated PAR sensor used on CTD casts in order to validate the attenuation coefficients estimated 
based on the VPR PAR sensor.

\section{Estimating Daily Nitrogen Fixation Rates}

The nitrogen-fixation-light model was used to estimate nitrogen fixation on the basis of gridded VPR Trichodesmium abundance data and estimated PAR. In order to calculate nitrogen fixation from the instantaneous irradiance model, PAR was simulated as described above at twelve minute intervals between sunrise and sunset.

The assumption was made that colonies were continuously randomly redistributed within the mixed layer, so that the average light exposure of a colony within the mixed layer would be approximately the average of light over the mixed layer. Under the simplifying assumption that a given colony spent equal time at all depths within the mixed layer, the irradiance input to the nitrogen fixation model within the mixed layer was the average of the modeled irradiance within that layer. Mixed layer depth is an indication of the past history of mixing in a given location, and does not necessarily indicate current active mixing to a given depth. However, it was the best indicator of the depth of mixing based on the available data.

Per-carbon nitrogen fixation rates were calculated for each time and grid position; these per-carbon rates were then multiplied by carbon biomass. Biomass was estimated by multiplying a morphology- and cruise-specific mean carbon content per colony by the number of colonies per volume at each grid location of the binned abundance data described above. Daily nitrogen fixation rates were estimated by summing hourly nitrogen fixation over the daylight period and multiplying by the time increment of $1 / 5$ hour.

Upper and lower bounds on the nitrogen fixation estimate are estimated by carrying out the same calculations using the high and low ends of the $95 \%$ confidence intervals for the model parameters and carbon per colony estimate. The resulting high-resolution estimates of nitrogen fixation are used to assess the vertical distribution of nitrogen fixation by Trichodesmium and the influence of physical processes on mesoscale nitrogen fixation variability. These nitrogen fixation estimates are com- 
pared with values from the database amassed by Luo et al. (2012) at similar locations (within $500 \mathrm{~km}$ of the cruise track) and times of year (within 90 year-days of the start and end of each cruise).

Where it is necessary to convert hourly measured nitrogen fixation rates to daily ones, nitrogen fixation has been assumed to vary sinusoidally over the light period based on a crude approximation of the modulation of light over the course of a day, following the approach of Davis and McGillicuddy (2006). Day length is calculated from latitude and day of year using a standard formula (Brock, 1981, Equation 4).

\subsection{Results and Discussion}

\subsubsection{PAR Estimation}

The attenuation coefficient was reasonably homogeneous throughout OC469 but higher in regions of low salinity for OC471 (Figure 4-3). Water associated with the freshwater plume observed on OC471 was turbid compared to the oceanic water observed elsewhere. The available PAR data were limited by a bias toward nighttime sampling and saturation of the PAR sensor at approximately $1200 \mu \mathrm{mol}$ quanta $\mathrm{m}^{-2} \mathrm{~s}^{-1}$, further limiting available daytime data.

The attenuation coefficients calculated from 5-m binned PAR show some variability with depth (Figure 4-4). However, this variability is similar in magnitude to variability among values at a single depth. Therefore, only limited improvement in accuracy of the light field could be gained through specification of depth-varying attenuation coefficients. The difference in attenuation coefficient in the freshwater region of OC471 is much larger in comparison.

A comparison between PAR modeled by the above method and in situ PAR measured by the CTD PAR sensor demonstrates broad agreement between the two (Figure 4-5). Perfect agreement between the observed values and those modeled based on climatological averages is not expected because of time-varying cloudiness. 
(a)

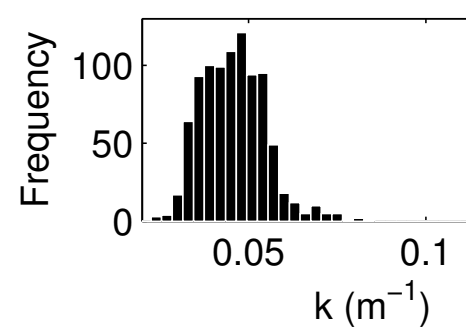

(c)

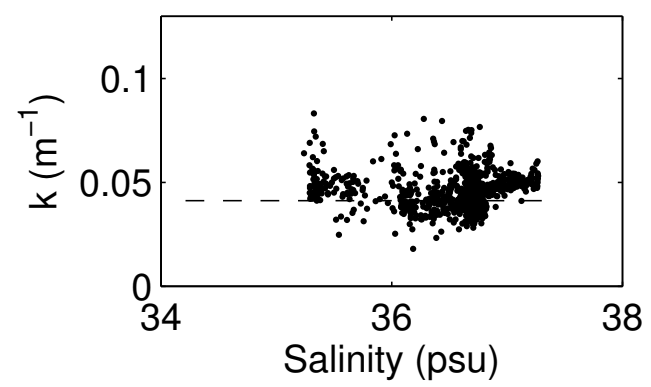

(b) OC471

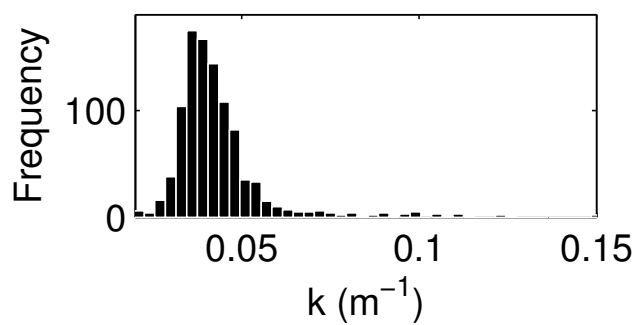

(d)

OC471

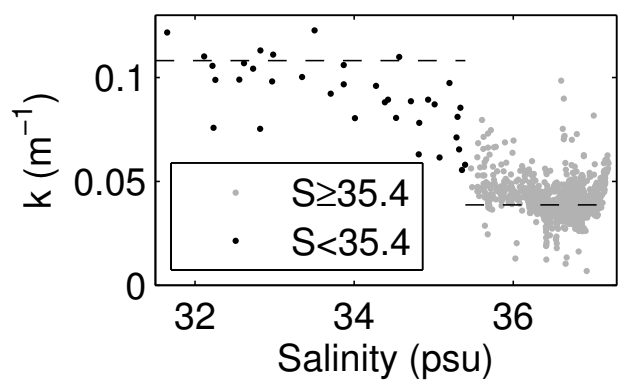

Figure 4-3: Vertical attenuation coefficients estimated from VPR PAR measurements and Eq. 4.6. Upper: Histograms display distributions from (a) OC469 and (b) OC471. Lower: Scatter plots of attenuation coefficient versus salinity for (c) OC469 and (d) OC471. Values grouped together for OC469. For OC471, attenuation coefficients were consistently higher in water with salinity less than 35.4. Dashed lines mark values that minimize mean squared error between modeled and observed PAR. These values are lower than the observed average values consistent with an observed tendency toward greater attenuation at depth, where PAR is less and therefore the effect on the mean square error is reduced. This is consistent with the pattern of greater attenuation with depth due to the changing angular distribution and upward scattering of downwelling irradiance for parallel incident light described by Kirk (1994, pp. 160-161). 


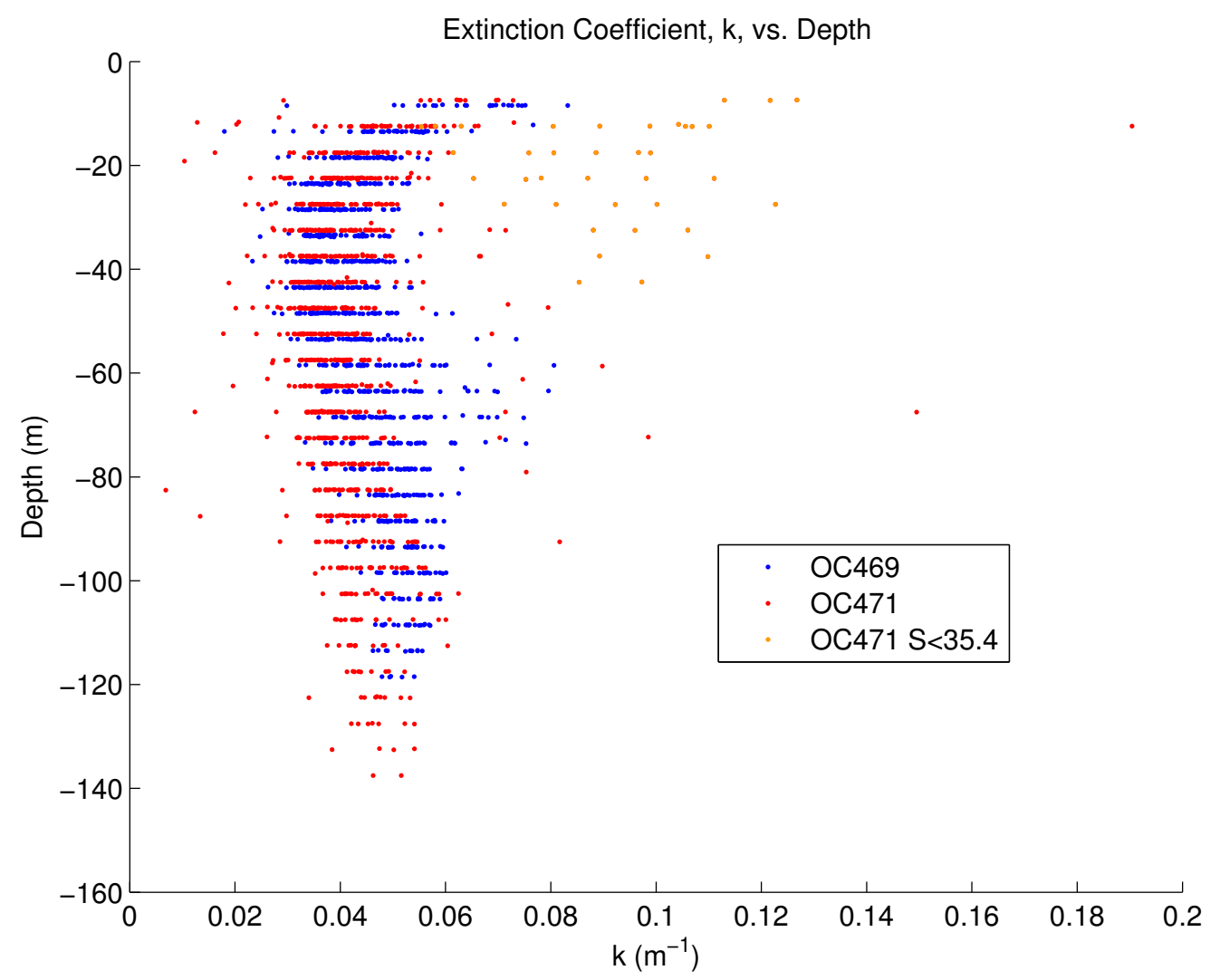

Figure 4-4: Depth distribution of vertical attenuation coefficients estimated from VPR PAR measurements and Eq. 4.6.

(a) OC469 Model vs. Observed PAR

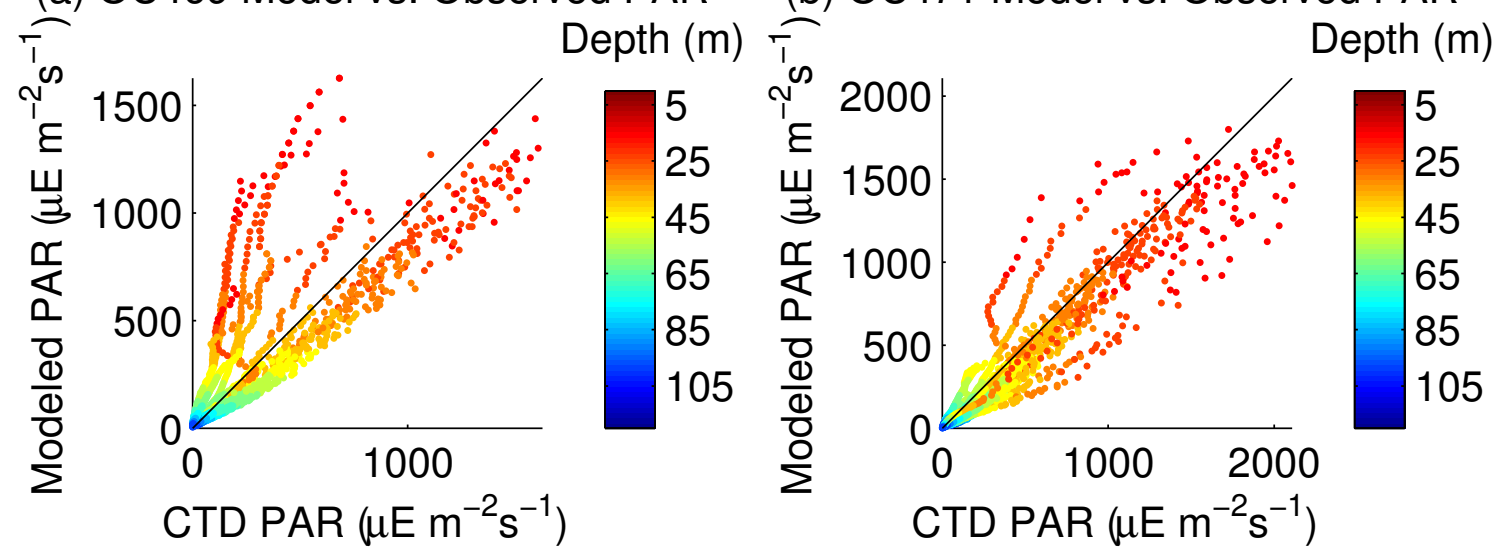

(b) OC471 Model vs. Observed PAR

Figure 4-5: Comparison of modeled to observed irradiance. Modeled irradiance is derived from SeaWiFS weekly climatological PAR estimates and Equation 4.7. Values at depth are computed using estimated mean extinction coefficients. PAR data from a recently calibrated sensor on the CTD deployed at stations are used for verification of the light model. 


\subsubsection{Relationship of Nitrogen Fixation with Temperature}

Previous studies have indicated that the effects of temperature may be primary drivers of Trichodesmium nitrogen fixation rates on a global scale. Trichodesmium nitrogen fixation has a narrow optimal temperature range, peaking at approximately $27{ }^{\circ} \mathrm{C}$ based on culture experiments (Breitbarth et al., 2007). A correlation between nitrogen fixation rate and temperature could not be assessed from OC469 or OC471 data because all incubations were carried out within a limited temperature range from 26.0 to $27.5^{\circ} \mathrm{C}$.

Temperature seems to be a primary driver of Trichodesmium biogeography. Application of the Breitbarth et al. (2007) temperature relationship to VPR temperature sections (Figure 4-6) shows that with few exceptions, Trichodesmium was most abundant within its optimal range for nitrogen fixation. Because Trichodesmium was consistently found near this optimal temperature for nitrogen fixation, we can conclude that over the majority of the cruise track, local variability in carbon-specific nitrogen fixation rates is driven by factors other than temperature dependence. Additionally, because Trichodesmium abundances are comparatively low in regions where temperature is suboptimal, the impact of temperature dependence on the volume-specific nitrogen fixation estimate would be small. Thus, while temperature effects on Trichodesmium physiology evidently shape the distribution of Trichodesmium colonies in this region, they can be ignored for the purposes of estimating nitrogen fixation in the favorable temperature areas where Trichodesmium was abundant.

Temperatures observed on OC471 are more favorable for nitrogen fixation than those from OC469. Based on Breitbarth's temperature-nitrogen-fixation curve, the reduction of nitrogen fixation rate from its maximum value due to temperature would be less than $20 \%$ for $86 \%$ of colonies observed on OC469 and $99 \%$ of colonies for OC471 (Figure 4-6c). The temperature effect would be less than $10 \%$ for $86 \%$ of

colonies from OC471. More favorable temperatures may have contributed to the greater abundances observed on OC471. 


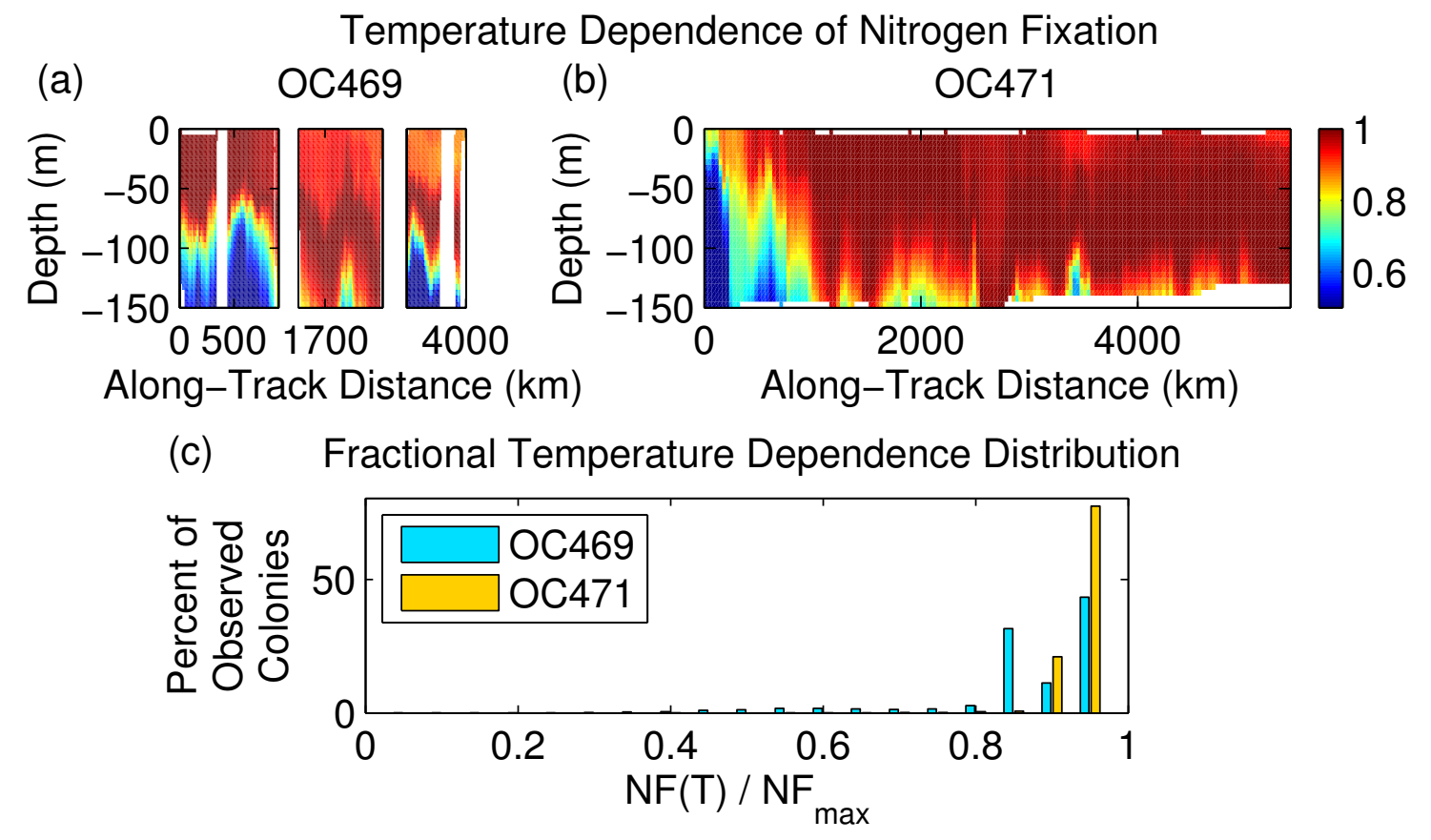

Figure 4-6: Dependance of nitrogen fixation rate on temperature based on the empirical function $N F=-0.001096 x^{2}+0.057 x-0.637$ (Breitbarth et al., 2007). Top panels display ratio of predicted to maximum $N_{2}$ fixation at temperatures observed on (a) OC469 and (b) OC471. For most regions sampled and nearly all parts of the upper water column where Trichodesmium is abundant, the ratio is close to one. (c) Histogram showing the percentage of colonies observed falling into various levels of temperature-dependence. Nearly all colonies fall into a small window of temperature dependence between factors of 0.8 and 1 . Therefore, temperature dependence may be expected to drive a small part of the observed nitrogen fixation variability.

\subsubsection{Nitrogen Fixation and Light}

In data from both cruises, POC-normalized nitrogen fixation linearly correlated with incubation irradiance at lower irradiance levels but leveled off at the higher end of the range of incubation irradiances tested (Figure 4-7). However, due to the limitations of the light sources in the incubators, most samples were incubated at relatively low levels of irradiance. Only a few samples from OC471 were incubated on deck in natural light. These samples at higher irradiances show that the linear relationship does not extend to high surface irradiance such as that observed close to the surface at midday. An extension of the linear relationship to the upper limit of field-observed irradiance would yield unrealistically high nitrogen fixation rates. The 
need for an upper bound on the nitrogen-fixation to incubation irradiance relationship is presented as justification for the choice of a saturating curve (Equation 4.3) rather than a simple linear relationship between nitrogen fixation and incubation irradiance.

The high light samples do not demonstrate photoinhibition. The two samples incubated at $1600 \mu \mathrm{mol}$ quanta $\mathrm{m}^{-2} \mathrm{~s}^{-1}$ fixed nitrogen at rates lower than the fit curve would predict; however, the rates were higher than any of those measured at the respective stations (13 and 16) at lower irradiances, which also tended to be low compared to the fit curve. The simple saturating curve (Equation 4.3) adequately captures the nitrogen-fixation-incubation irradiance relationship to the extent it is resolved by the available data.

In addition to incubation light level, carbon-specific nitrogen fixation rates correlated with estimated in situ daily PAR. This relationship is roughly consistent with modeled nitrogen fixation rates integrated over the course of a day (Figure 4-8).

Measurements from both cruises were used to derive the relationship between nitrogen fixation and light even though there were some methodological differences on the two cruises. On OC469 a pump sampling method for surface and deep samples resulted in smaller volumes sampled and less biomass available for nitrogen fixation experiments compared to the MOCNESS sampling on OC471. However, some samples were taken from near-surface hoop net tows on OC469. The net tows, despite sampling over a broader depth range, had nitrogen fixation rates similar to or higher than surface pump samples. It is possible that the pump-derived rates are artificially low, perhaps as a result of the smaller volume of water sampled resulting in a decreased likelihood of encountering healthy, active colonies or due to disruption of the colonies. However, nitrogen fixation rate did not scale with the carbon biomass per sample except for a few of the smallest samples. The lower tendency of nitrogen fixation rates on the fall cruise OC469 compared to the spring cruise OC471 may be the result of true temporal, possibly seasonal, variability. Despite this apparent variation in the magnitude of nitrogen fixation between the two cruises, including data from both cruises in the model fits reduced the parameter uncertainty as diagnosed by the 

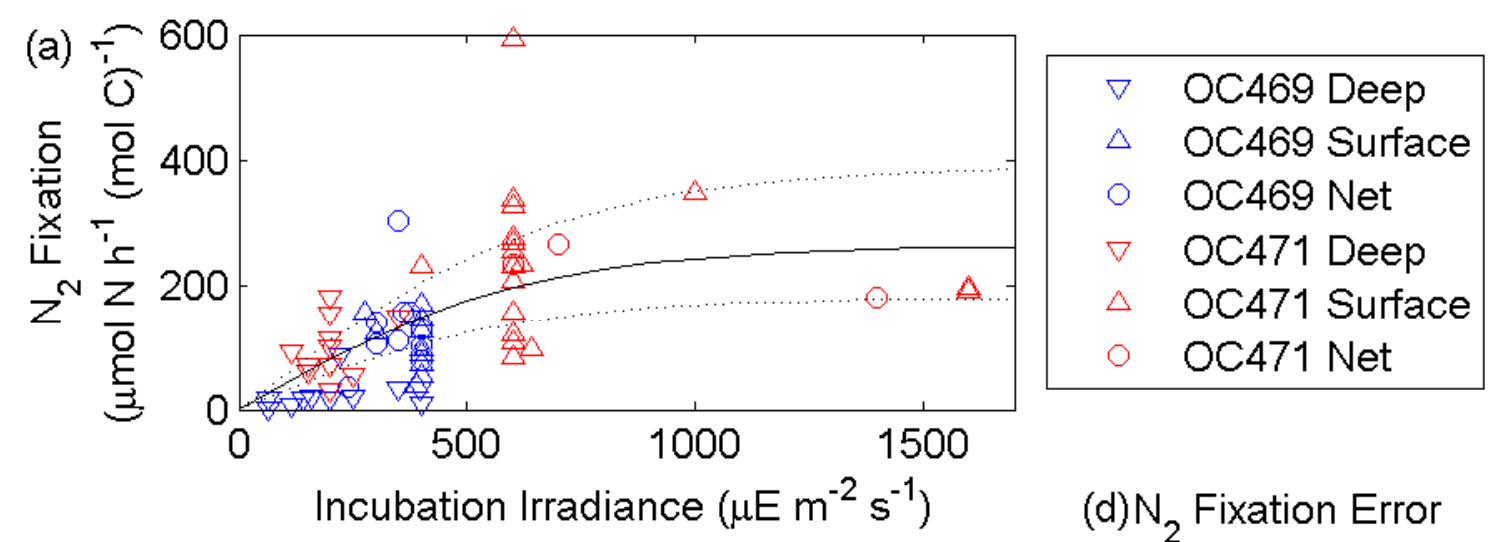

$\begin{array}{lll}\text { (b) Profile Log Likelihood } & \text { (c) } 95 \% \text { Confidence Regions }\end{array}$
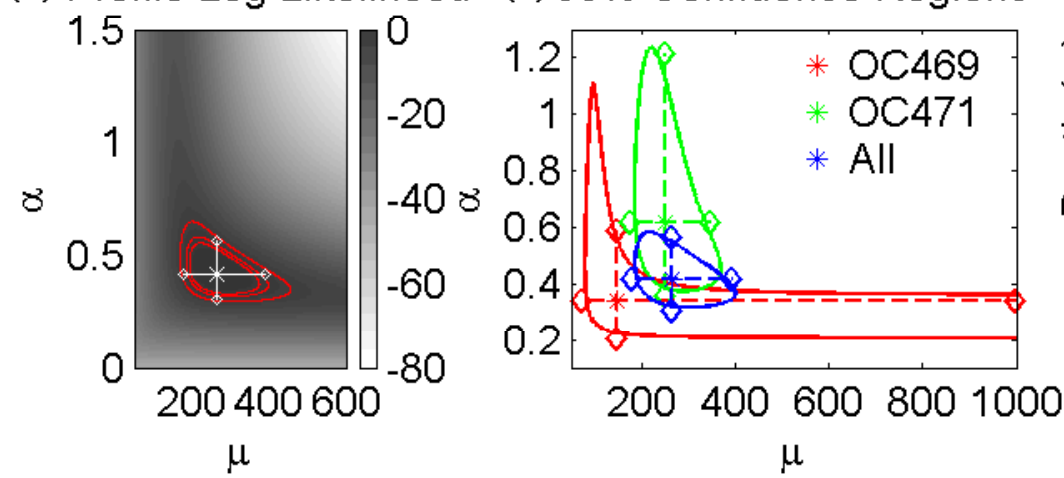

(d) $\mathrm{N}_{2}$ Fixation Error

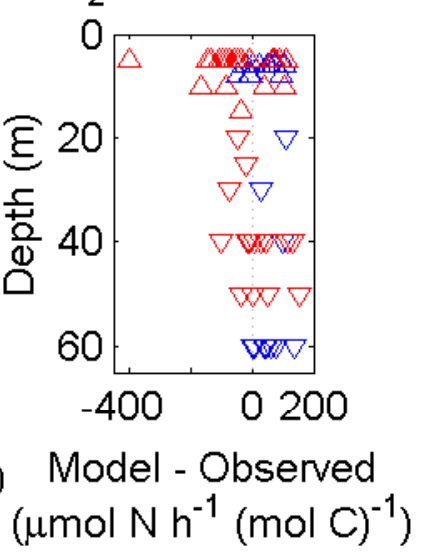

Figure 4-7: (a) Nitrogen fixation as a function of incubation irradiance. Symbols differentiate cruises and colony sources (surface, deep, or net tow). A solid black line represents the least squares fit to Eq. 4.3, based on all available data from OC469 and OC471. Dashed lines indicate upper and lower bounds based on profile log likelihood $95 \%$ confidence intervals for $\alpha$ and $\mu_{\max }$. (b) Profile log likelihood of observations based on model Eq. 4.3 and normally distributed error. Asterisk indicates maximum likelihood estimate for all parameters $(\sigma, \alpha$, and $\mu$ ), while red contours indicate 90 , 95, and 99\% confidence regions. White lines demarcate profile log likelihood 95\% confidence intervals for each parameter individually, with the others at maximum likelihood estimates. Units for $\mu_{\max }$ and $\alpha$ are $\mu \mathrm{molN}(\mu \mathrm{molC})^{-1} \mathrm{~h}^{-1}$ and $\mu \mathrm{mol} \mathrm{N}$ $(\mu \mathrm{mol} \mathrm{C})^{-1} \mathrm{~h}^{-1} \mu$ Einsteins ${ }^{-1} \mathrm{~m}^{2} \mathrm{~s}$. (c) Maximum likelihood estimate and $95 \%$ confidence regions based on profile log likelihood for subsets of data. (d) Model-data misfit as a function of depth with symbols as in (a). 


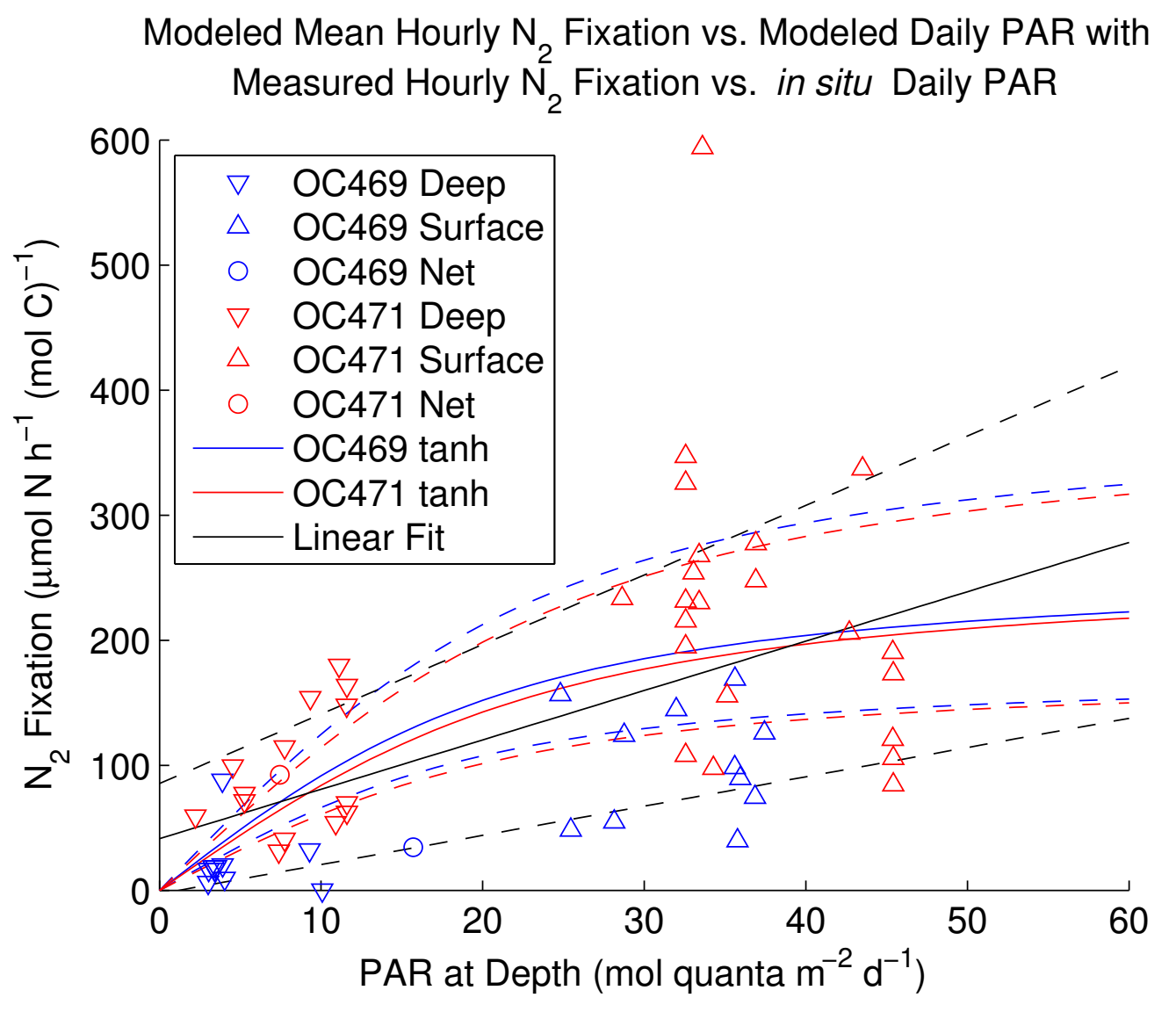

Figure 4-8: Measured nitrogen fixation rates vs. in situ Irradiance with linear regression (black line) and 95\% confidence intervals (black dashed lines). Also shown: Daily mean modeled nitrogen fixation rates, calculated by dividing daily rates by the number of hours of daylight. Dashed lines delimit fits based on parameter 95\% confidence intervals. 
breadth of $95 \%$ confidence intervals.

Detailed examination of the nitrogen-fixation-irradiance fit reveals some systematic errors. Nitrogen fixation was higher on OC471 than OC469, so the fit tends to overestimate OC469 values and underestimate OC471 values. Additionally, surfacecollected samples tend to have higher rates than deep samples at similar incubation light levels, particularly for OC469. Also, there is a slight tendency for the model to underestimate surface nitrogen fixation and overestimate deep nitrogen fixation, particularly for OC469 (Figure 4-7). Thus, the model may systematically over- or under-estimate nitrogen fixation in certain conditions. It is important to realize that there are many other sources of variability in nitrogen fixation rates that are not considered in the light-limited model, such as iron and phosphorus availability and temperature.

An additional complication arising in the modeling of nitrogen fixation rates based on irradiance is diel variation. While diel variation in Trichodesmium nitrogen fixation has been attributed to a circadian rhythm that can continue for some time even in the absence of a light/dark cycle (Chen et al., 1996), the observed diel variation in the natural environment is broadly consistent with the modulation of irradiance over the course of a day. For example, the daily nitrogen fixation curve presented by Saino and Hattori (1978)'s Figure 1 has a shape similar to a daily irradiance curve, with maximum at noon and approaching zero at sunrise and sunset. Diel variability is built in to the model due to variation in the input irradiance parameter over the course of the day. Thus, the instantaneous response of nitrogen fixation to irradiance in this model provides a natural way to simulate diel variability of Trichodesmium nitrogen fixation.

Mechanistically, the relationship between nitrogen fixation and light is complex. Photosynthesis provides both the energy and reductant required for nitrogen fixation. However, oxygen evolved during photosynthesis can render the nitrogenase enzyme ineffective, driving most nitrogen-fixing cyanobacteria to spatially or temporally segregate photosynthesis from nitrogen fixation. Trichodesmium, in contrast, is 
nonheterocystous and fixes nitrogen during the day concurrent with photosynthesis. In fact, nitrogen fixation does not occur in Trichodesmium in the absence of light; although Trichodesmium has been demonstrated capable of fixing nitrogen using respiratory electrons alone, the electrons are not supplied to the ferredoxin-nitrogenase pathway in the absence of the light-dependent action of photosystem I (Wilson et al., 2012). Energy for nitrogen fixation can also be supplied via photosystem I through ATP from cyclic electron flow and the Mehler reaction, in which oxygen is reduced, potentially mitigating oxygen toxicity (Berman-Frank et al., 2007).

Modeling nitrogen fixation as a function of instantaneous irradiance assumes immediate adjustment to changing light levels. This simplest case is assumed based on the absence of nitrogen fixation at zero irradiance and the diel variation observed by Saino and Hattori (1978). However, some studies have demonstrated a latent effect of previous light exposure on nitrogen fixation rates. Heithoff (2011) reported that nitrogen fixation rates decreased when cultures grown at higher irradiance were shifted to lower irradiance, with most of the adjustment taking place within the first twentyfour hours of the experiment. Wilson et al. (2012)'s manipulation experiments on cellular response to increasing irradiance demonstrated higher maximum $\mathrm{H}_{2}$ production (a product of nitrogen fixation), electron transport rate, and nitrogenase activity (acetylene reduction) in samples held at $500 \mu \mathrm{mol}$ quanta $\mathrm{m}^{-2} \mathrm{~s}^{-1}$ for five days com-

pared to those grown at $50 \mu \mathrm{mol}$ quanta $\mathrm{m}^{-2} \mathrm{~s}^{-1}$. It is possible that the nitrogen fixing capacity, represented in our model by $\mu_{\max }$, may adjust gradually to changes in light conditions, while the response below saturating irradiances, represented by $\alpha$, reflects the dependence on energy supply from photons.

\subsubsection{Carbon Per Colony}

Variations in colony carbon content with depth were insignificant compared to uncertainty in the means (Figure 4-9). Means, standard deviations, and 95\% confidence intervals were calculated on log-transformed carbon per colony measurements (Table 4.2). Mean carbon per colony was higher for OC471 than for OC469; a Stu- 


\begin{tabular}{lccc}
\hline Group & Mean & Standard Deviation & $95 \%$ Confidence Interval \\
\hline OC469 & 0.52 & 1.79 & $0.16-1.64$ \\
OC469 puffs & 0.31 & & $0.13-0.49$ \\
OC469 rafts & 0.67 & 1.61 & $0.56-0.78$ \\
OC471 & 0.74 & & $0.29-1.91$ \\
OC471 puffs & 0.62 & & $0.45-0.78$ \\
OC471 rafts & 0.94 & 1.75 & $0.82-1.06$ \\
Combined & 0.62 & & $0.20-1.87$ \\
Combined puffs & 0.55 & & $0.42-0.67$ \\
Combined rafts & 0.84 & & $0.75-0.92$ \\
\hline
\end{tabular}

Table 4.2: Carbon per colony ( $\mu$ mol $\mathrm{C}$ colony $^{-1}$ ) estimated from log-transformed means or linear regressions.

dent's t-test demonstrates that the means are different at the $5 \%$ significance level $(\mathrm{p}=9 \mathrm{e}-5)$. Multiple linear regressions of carbon content versus number of puffs and number of rafts for the two cruises estimated morphology-specific carbon per colony (Table 4.2). For both cruises, rafts tended to have higher carbon content than puffs.

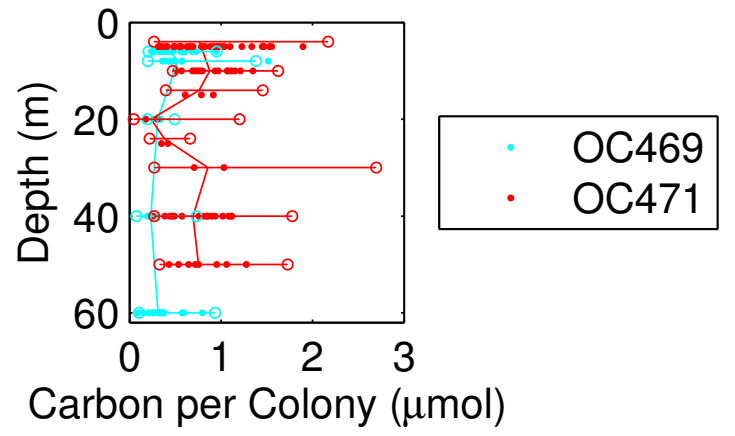

Figure 4-9: Carbon per colony vs. depth. Means (based on log-transformed data) within $5 \mathrm{~m}$ depth intervals are connected by solid lines as are edges of $95 \%$ confidence intervals, which are demarcated by circles.

\subsubsection{Model Assessment}

In order to assess the level of uncertainty in the nitrogen fixation estimates, we present an example of Trichodesmium nitrogen fixation fields calculated using parameter values at the upper and lower bounds of their 95\% confidence intervals, as 
described in Section 4.3.2 (Figure 4-10). On average, upper and lower bounds on integrated nitrogen fixation rates were 0.60 and 1.66 times the best estimates in this section.

Modeled nitrogen fixation rates were roughly consistent with rates estimated directly from incubation experiments under an assumption of sinusoidal modulation of nitrogen fixation throughout the light period (Figure 4-11). Modeled rates are based on climatological light levels at the depth of collection. These were not always consistent with actual incubation light levels of the samples. Therefore, variability between modeled and measured rates is expected. Rates calculated based on observed light profiles and the Davis and McGillicuddy (2006) model (based on percent of surface irradiance rather than absolute PAR) more strongly over- or under-estimate the observed nitrogen fixation rate compared to the OC469-OC471 model fit to the new nitrogen fixation rates.

Modeled rates reflect patterns in abundance and light. However, perhaps the largest source of uncertainty is variability in nitrogen fixation rates arising from processes that were not modeled. For instance, iron and phosphorus availability could affect the physiological state of the colonies and their nitrogen fixation rates. Actual nitrogen fixation patterns could differ from those simulated due to the effects of these drivers on per-carbon nitrogen fixation rates. These drivers can affect bulk nitrogen fixation rates through impacts on per carbon Nitrogen fixation rates and through their effects on biomass. The latter are accounted for through the direct measurement of biomass, but the former are not.

Another source of uncertainty is nitrogen fixation by free filaments. Whereas nitrogen fixation assays were carried out only on the more readily sampled colonial morphologies, free filaments were also present everywhere Trichodesmium was abundant. Free filament biomass was calculated for stations where microscope filament counts were available. We assumed a ratio of $3.091 \mathrm{nmol}$ carbon per filament, based on the average carbon per colony from the two cruises of $0.6182 \mu \mathrm{mol} \mathrm{C}$ per colony and an assumed filament content of 200 per colony, as in Luo et al. (2012). The 


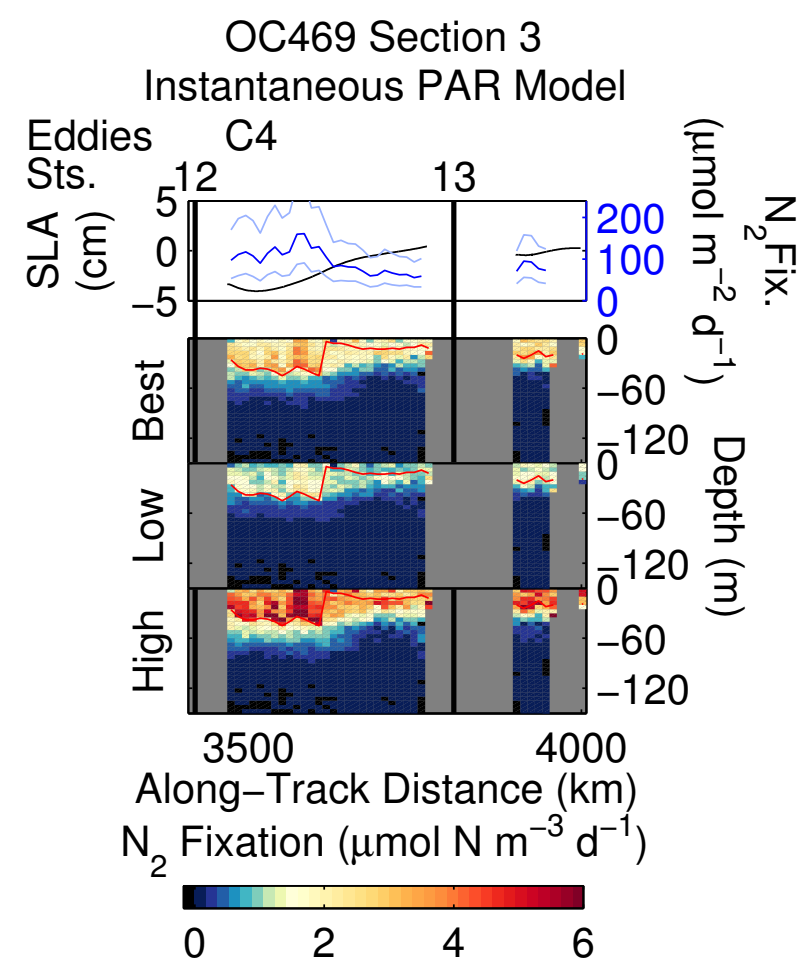

Figure 4-10: Nitrogen fixation estimates for OC469 VPR Section 3. "Best" panel uses maximum likelihood estimates for model parameters $\left(\mu_{\max }\right.$, and $\left.\alpha\right)$ and carbon per colony estimates for puffs and rafts; "Low" panel uses parameter values based on lower bounds of 95\% confidence intervals; and "high" panel uses parameters and puff and raft carbon per colony estimates based on upper bounds of $95 \%$ confidence intervals. In upper panel, the black line represents sea level anomaly and the blue lines represent depth-integrated nitrogen fixation based on parameter values at their upper and lower $95 \%$ confidence intervals.

carbon content of free filaments is assumed the same as that of colonial filaments. Free filaments made up approximately $6.7 \%$ of Trichodesmium biomass on OC469 and $9.8 \%$ on OC471, or $8.2 \%$ overall based on pooled data from both cruises (Figure 4-12). The fraction was reasonably consistent across cruises and abundance levels, and was similar to previously reported levels in the region. Carpenter et al. (2004) reported cruise free filament fraction ranging from 7 to $11 \%$ in the tropical North Atlantic. To estimate total nitrogen fixation including fixation by filaments, one might multiply the rates presented below by 1.09 (the mean ratio of free to colonial filaments, corresponding to a free filament biomass fraction of $8.2 \%$, is 0.09 ). However, 


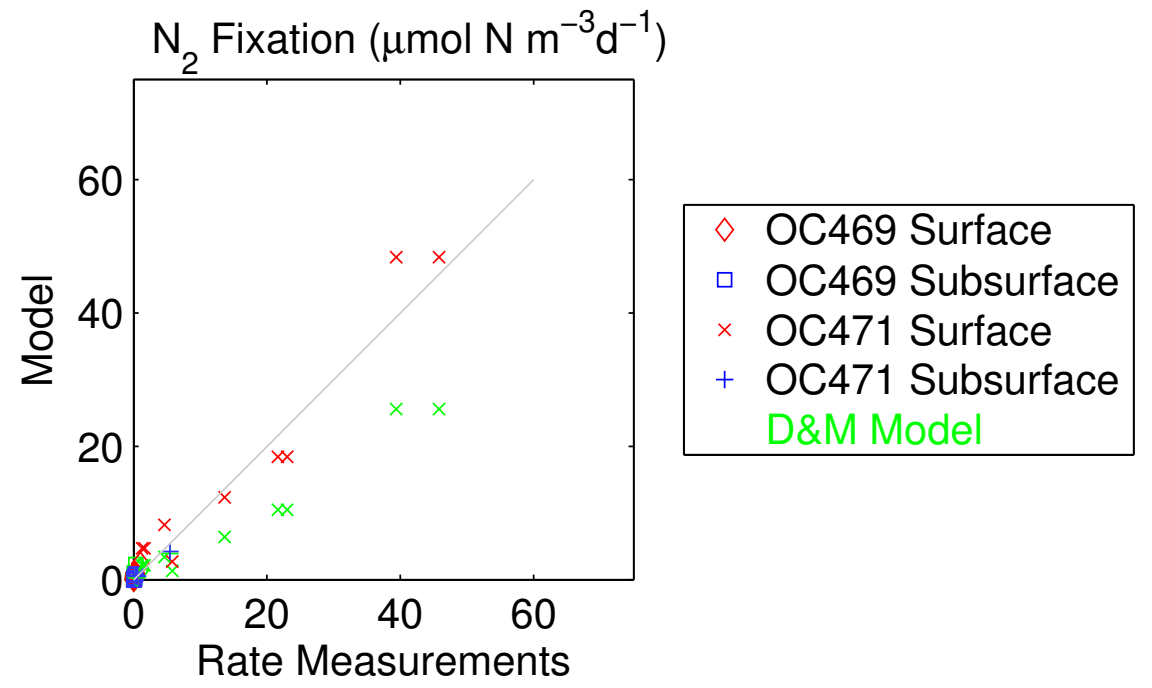

Figure 4-11: Comparison between modeled Trichodesmium nitrogen fixation rates (using the model presented here and the Davis and McGillicuddy (2006) model) and rates estimated from incubations under the assumption of constant rates throughout the daylight period. 
as rates of nitrogen fixation by free filaments were not measured, such a correction is not made.

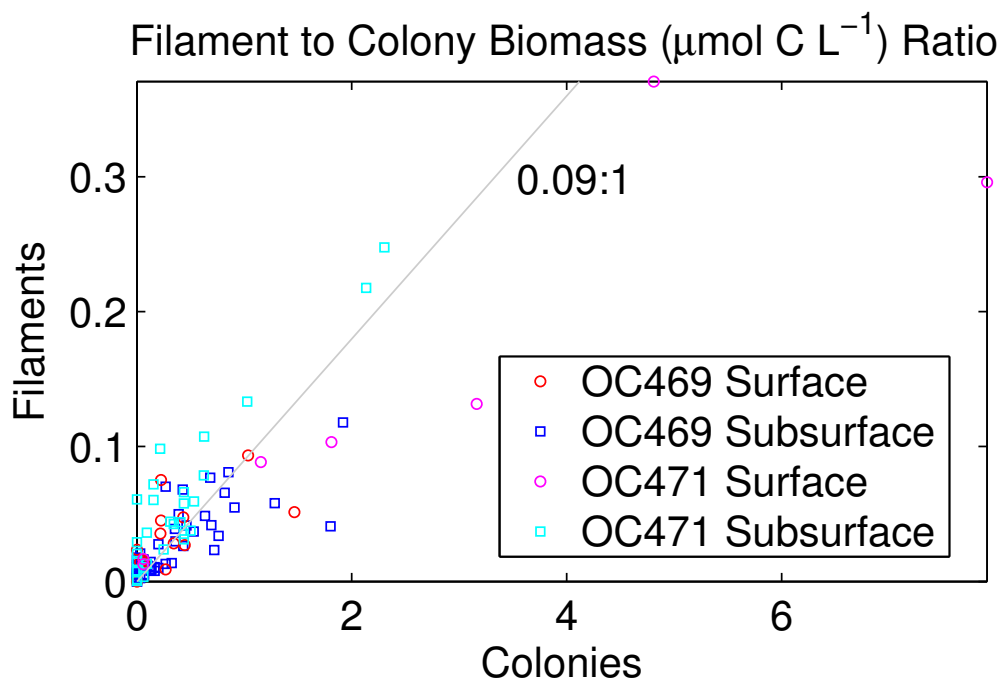

Figure 4-12: Estimated contribution of free filaments to total Trichodesmium biomass.

\subsubsection{Mesoscale to Sub-Basin-Scale Estimates of Trichodesmium Biomass and Nitrogen Fixation}

Consistent with previous findings, estimated nitrogen fixation rates were on average highest at the surface, decreasing with depth (Figure 4-13). Profiles of nitrogen fixation versus depth vary depending on the distribution of colonies, but maxima are consistently present in the upper $20 \mathrm{~m}$ due to the effect of irradiance. Mean vertical profiles of modeled nitrogen fixation were not inconsistent with those calculated from data compiled by Luo et al. (2012) (Figure 4-13). Thus, the canonical view of strongly surface-intensified Trichodesmium nitrogen fixation is upheld in the mean in this region, with $91 \%$ of nitrogen fixation occurring above $45 \mathrm{~m}$ depth and $65 \%$ in the upper $20 \mathrm{~m}$ in the mean profile.

Application of the model to the entire VPR abundance data set provides an opportunity to analyze nitrogen fixation on a finer scale than is typically observed. Lateral variability in modeled nitrogen fixation is strongly associated with variation in Tri- 
chodesmium biomass. For instance, local peaks in biomass associated with cyclonic eddies along VPR Section 2 of OC469 were also local maxima in modeled nitrogen fixation rate (Figure 4-14). However, some subsurface features of Trichodesmium biomass translate to less intense features in modeled nitrogen fixation due to the dependence of nitrogen fixation on light. For instance, the elevated biomass signals at depth in OC469 cyclone C4 (Figure 4-14, upper right) and OC471 anticyclone A4 (Figure 4-15, upper middle) correspond to comparatively damped nitrogen fixation signals at depth. Additionally, the highest integrated biomass observed did not correspond to the maximum modeled nitrogen fixation rate due to the greater extinction of light in that region, despite the relatively shallow distribution of biomass there (Figure 4-15, section 2). Estimated nitrogen fixation generally reflected patterns in biomass with a skew toward greater response near the surface due to higher PAR levels there.

Trichodesmium biomass covaried with physical properties on both cruises (Figures 4-14 and 4-15, top panels). Total Trichodesmium biomass, dominated by the more buoyant raft morphology, was markedly elevated within the mixed layer compared to the waters below over much of the area sampled on both cruises. For example, compare the deep depth distribution of biomass in OC471 anticyclone A4, which had a deep mixed layer, to the shallow depth distribution in anticyclone A5, which was associated with a low salinity lens confined to surface waters (Figure 4-15, upper middle). The exception to this pattern was part of the southernmost region sampled on OC471 (Figure 4-15, section 3 and part of section 2), where biomass remained at relatively high levels for some distance below the shallow mixed layer. Elsewhere, gradients in biomass, particularly of rafts, tended to coincide with gradients in density, suggesting that stratification may act as a barrier to the mixing of buoyant rafts deeper in the water column.

In addition to stratification, Trichodesmium biomass and nitrogen fixation also demonstrated a relationship with salinity on the southern section of OC471. The two regions of highest biomass and modeled nitrogen fixation were associated with low 
salinity, likely of riverine origin. These high biomass, low salinity features were in an anticyclone with a deep mixed layer (A4) and a low-salinity lens (encompassing anticyclone A5) sampled on OC471 (Figure 4-15). Amazon river freshwater input was suggested as an explanation for elevated phytoplankton abundance tied to nitrogen fixation in this region by Coles et al. (2004). Subramaniam et al. (2008) also described the contribution to diazototrophic activity by outflow from the Amazon and Orinoco rivers transports nutrients far into the tropical Atlantic. Thus, the co-occurence of elevated Trichodesmium biomass with anomalously fresh water may be related to nutrient input through river outflow.

The VPR data provide the opportunity to assess mesoscale variations in abundance and nitrogen fixation by colony morphology. Raft biomass and nitrogen fixation were consistently higher than those of the other morphologies throughout both cruises. However, toward the southern end of the domain, the puff and bowtie morphologies contributed a larger fraction of the total biomass than elsewhere (Figure 4-14, section 3, and Figure 4-15, section 3; also, compare eddies C1 and C3 with eddy C4 in Figure 4-16). Throughout the southern portion of OC471, the puff and bowtie morphologies exhibited nearly as high biomass and nitrogen fixation as rafts (Figure 4-15, right panels). In this region, the three morphologies had nearly identical depth-distributions, but with slightly different magnitudes.

Raft colony biomass was greatest within the mixed layer throughout OC469 and most of OC471 and was more strongly surface-intensified than puff or bowtie biomass. On OC469, mean puff biomass actually peaked subsurface, on average, due to a region of elevated puff abundance just below the mixed layer in the southernmost section (Figure 4-14, section 3). In the northern part of the study area, low levels of puff biomass were uniformly distributed with depth throughout the upper $150 \mathrm{~m}$ sampled, consistent with conditions observations over parts of the transatlantic VPR survey by Davis and McGillicuddy (2006) and the western Caribbean survey of Davis et al. (2013). In contrast, in the freshwater-influenced southern portion of OC471, vertical distributions were consistent across morphologies (Figure 4-15, sections 2 and 3). 
In this region, the three morphologies had nearly identical depth-distributions, with slightly different magnitudes, and the relationship between Trichodesmium biomass and the mixed layer observed elsewhere was not evident.

Puff biomass peaked below the base of the mixed layer in cyclone C4 on OC469 (Figure 4-14). Possibly these puffs are the remnants of a bloom that initiated closer to the surface and sank. However, it seems more likely that these puff colonies are adapted to thrive at these depths. Although the feature is unique within the observations from OC469 and OC471, puff colony abundances have been observed to peak at depth in some locations during other studies, such as the western subtropical Atlantic (Davis and McGillicuddy, 2006), part of a transect in the western Caribbean (Davis et al., 2013), and in the Gulf of Aqaba in the Red Sea (Post et al., 2002). This is consistent with findings that rafts are typically more buoyant than puffs (Walsby, 1978). The subsurface puff abundance feature in OC469 cyclone C4 was the most dense aggregation of puffs observed on OC469. Extending over a distance of only approximately $100 \mathrm{~km}$ along the cruise track, it could easily have been missed by more discrete sampling methods such as net tows.

In OC469 cyclone C4 from the present study as well as the western Caribbean section (Davis et al., 2013) and parts of the transatlantic section (Davis and McGillicuddy, 2006), puffs were the locally dominant colony morphology. However, these local maxima in puff colony abundance were small compared to the highest raft abundances observed elsewhere. In the present study, even though the local peak in puff colony abundance was slightly higher than the local peak in raft abundance, it translated to a slightly smaller peak in biomass compared to the rafts biomass due to the smaller average carbon content of puff colonies.

The location of the peak below the mixed layer in low light conditions may indicate that puffs fill a different niche from the surface-dwelling rafts, as suggested by Post et al. (2002). Thus, it is possible that this atypical feature in the puff distribution may also have unique physiological characteristics reflecting low light adaptation. Physiological adaptations to low irradiance have been observed in Trichodesmium in 
culture, including increased cell diameter for light absorption and changes in coupling of phycobilisomes with the photochemical reaction centers (Andresen et al., 2010). If, in fact, this population exhibits anomalous nitrogen fixation rates, the effect cannot be captured by this study. The nitrogen fixation model is based largely on nitrogen fixation rate measurements dominated by colonies of the more abundant raft morphology. No nitrogen fixation samples were taken from within this subsurface puff maximum. Estimated nitrogen fixation rates there peaked deeper in the water column than at other locations and were the highest observed on OC469 due to a combination of elevated puff and raft biomass. The potential of this type of subsurface puff feature to influence nitrogen fixation rates warrants further investigation through direct rate measurements, and the VPR is an ideal tool to identify potential sampling sites.

Although puffs have at times been observed to be more deeply distributed than rafts, this was not the case in the lower salinity waters in the southern region sampled during OC471. Thus, the presence of relatively large numbers of puff and bowtie colonies is not necessarily indicative of enhanced nitrogen fixation at depth. In the freshwater lens observed on OC471, puff colonies were abundant, but stratification was high near the surface due to the salinity gradient. There, nitrogen fixation was more strongly surface-intensified than anywhere else on the cruise. This is explained by both the location of colonies within the thin surface layer and the rapid extinction of light in the turbid riverine surface water, possibly due to dissolved organic matter of riverine origin or biological material originating from nutrients supplied through the river plume. Overall, the stratification environment emerges as the most important factor in determining the distribution of colonies with depth, and in turn, inferred nitrogen fixation patterns.

In summary, although in the mean profile, nitrogen fixation rates decay significantly with depth in the upper 50 meters (Figure 4-13), elevated nitrogen fixation rates are calculated as deep as $100 \mathrm{~m}$ in certain locations, such as OC469 eddy C4 and OC471 eddy AC4 (Figure 4-16). Those two deep nitrogen fixation sites together comprised approximately $700 \mathrm{~km}$ (roughly 10\%) of the total track length sampled by 
the VPR. Similar regions of deep Trichodesmium abundance, and presumably deep N-fixation, were encountered by Davis and McGillicuddy (2006) in the western North Atlantic,Davis et al. (2013) in the western Caribbean, and Post et al. (2002) in the Gulf of Aqaba. The two regions of relatively deep biomass and nitrogen fixation from the present study occurred in the southern portion of the study region, at latitudes where freshwater riverine influence was present. However, while salinity in OC471 anticyclone AC4 was anomalously low, salinity was consistent with the surrounding oceanic water in OC469 cyclone C4. In both features, deep nitrogen fixation was associated with deeper than average mixed layers, suggesting that a deep mixed layer may be requisite for deep nitrogen fixation at these latitudes. This may be attributable to deeper mixing of buoyant Trichodesmium colonies in well-mixed surface layers where there is less stratification to act as a barrier to the downward propagation of turbulent motions. However, in C4, puffs peaked below the mixed layer. Nonetheless, it may be helpful in future modeling of Trichodesmium nitrogen fixation rates to include dynamical interactions among buoyant Trichodesmium, stratification, and mixing.

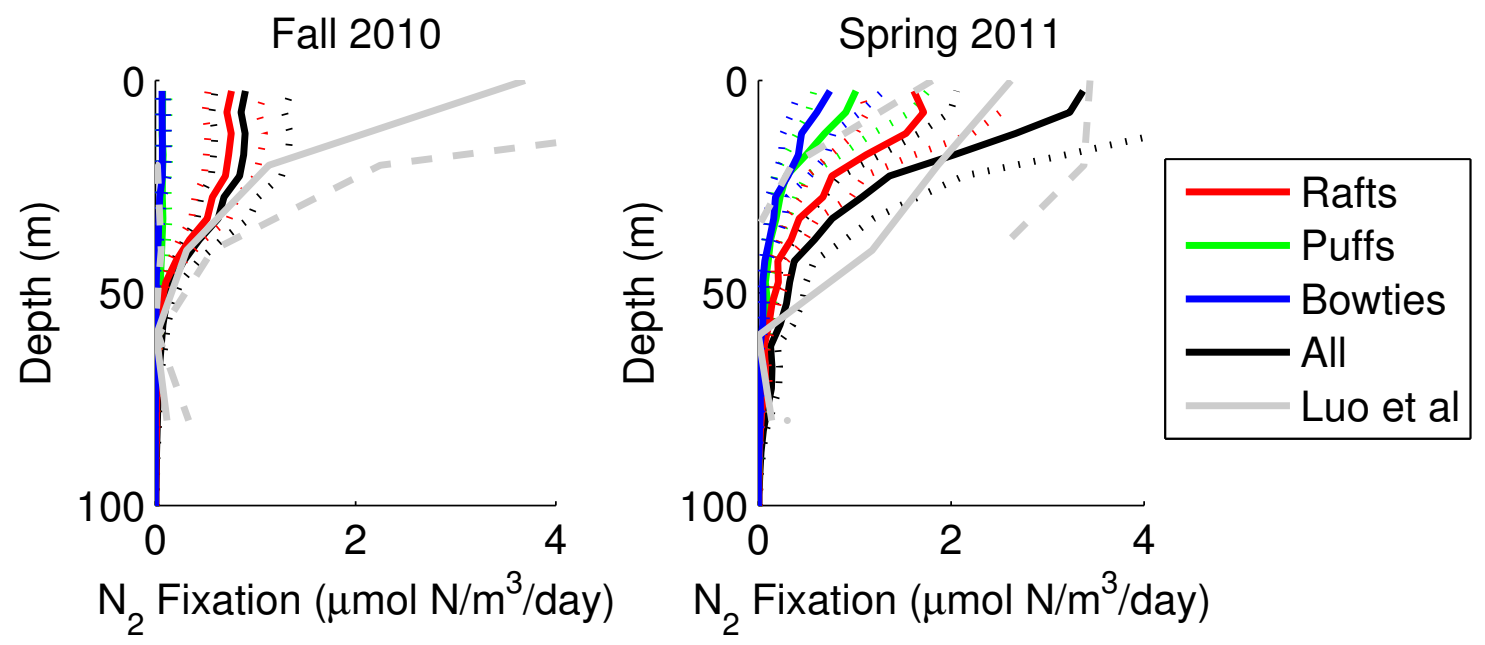

Figure 4-13: Nitrogen fixation versus depth for the two cruises. Dashed lines represent upper and lower bounds based on parameters' 95\% confidence intervals. Gray line is mean of depth-binned values from Luo et al. (2012) dataset for similar location and season to VPR track, with gray dotted lines representing $95 \%$ confidence intervals. 


\subsubsection{Large-scale Spatial Variations in Nitrogen Fixation: Comparisons with Luo et al. (2012) and Davis and McGillicuddy (2006)}

VPR-based Trichodesmium colony abundance and modeled nitrogen fixation rates were compared with data extracted from the set of conventionally sampled observations compiled by Luo et al. (2012) in corresponding geographic domains (Figures 4-17 and 4-18). The conventional methods used to collect the data included in the Luo et al. (2012) database were light microscopy on samples collected by net or Niskin bottle for abundances, and acetylene reduction or ${ }^{15} \mathrm{~N}_{2}$ assimilation experiments for nitrogen fixation. VPR-based abundance profiles from Davis and McGillicuddy (2006) are reproduced for comparison (Figure 4-18). Additionally, rates estimated using an adapted version of the nitrogen fixation model used by Davis and McGillicuddy (2006) are compared to rates based on the present model and from Luo et al. (2012) (Figure 4-18). The Davis and McGillicuddy (2006) model was adapted by scaling the surface maximum modeled per colony nitrogen fixation rates by a factor of 0.16 to reflect mean surface rates observed in the present study.

Depth-integrated abundances and nitrogen fixation rates from OC469 and OC471 fall within the range of conventionally-derived estimates, although the more spatially and temporally diverse data from Luo et al. (2012) exhibit greater variability and higher peak values (Figure 4-17). Integrated nitrogen fixation rates from the present study tend to be slightly lower than the corresponding rates from Luo et al. (2012), particularly those from OC469. Given the degree of variability among nearby measurements in the Luo et al. (2012) dataset, it seems reasonable to attribute these differences to temporal variability. Broad geographical patterns are similar, with nitrogen fixation increasing from subtropics to tropics and peaking in the southwestern North Atlantic in both spring and fall. The regions of peak abundance and nitrogen fixation are consistent with the regions of freshwater influence observed on the two cruises. Thus, freshwater input, possibly a source of nutrients due to river outflow 

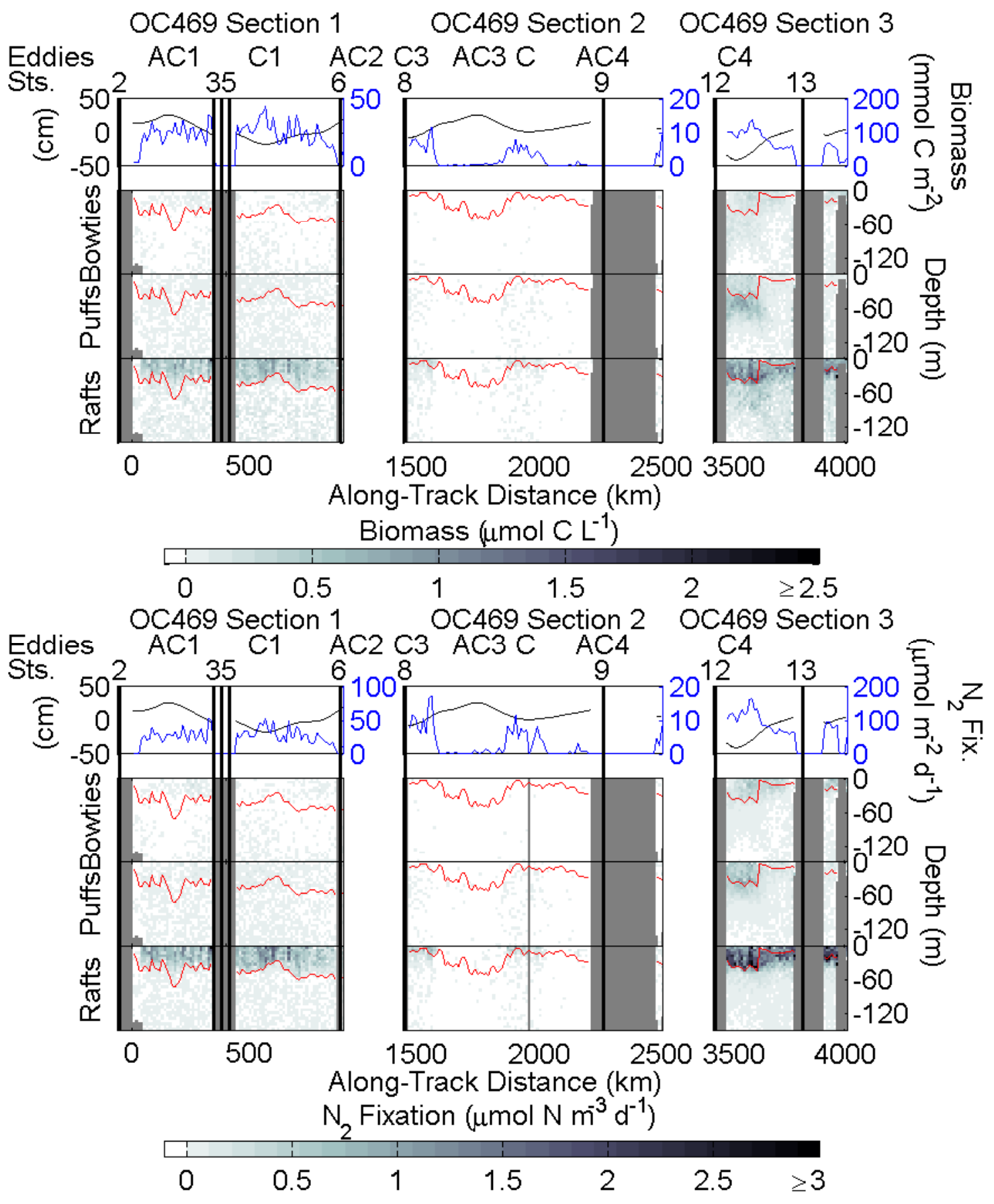
Figure 4-14: OC469 Trichodesmium biomass (upper) and modeled nitrogen fixation estimates (lower). For each section, the top panel displays sea level anomaly (black) and depth-integrated biomass/nitrogen fixation (blue). The lower three panels show biomass or nitrogen fixation by colony morphology. Black vertical lines mark station locations. Red lines designate mixed layer depth. Gray areas are regions not sampled by VPR. 
OC471 Section $1 \quad$ OC471 Section 2 OC471 Section 3

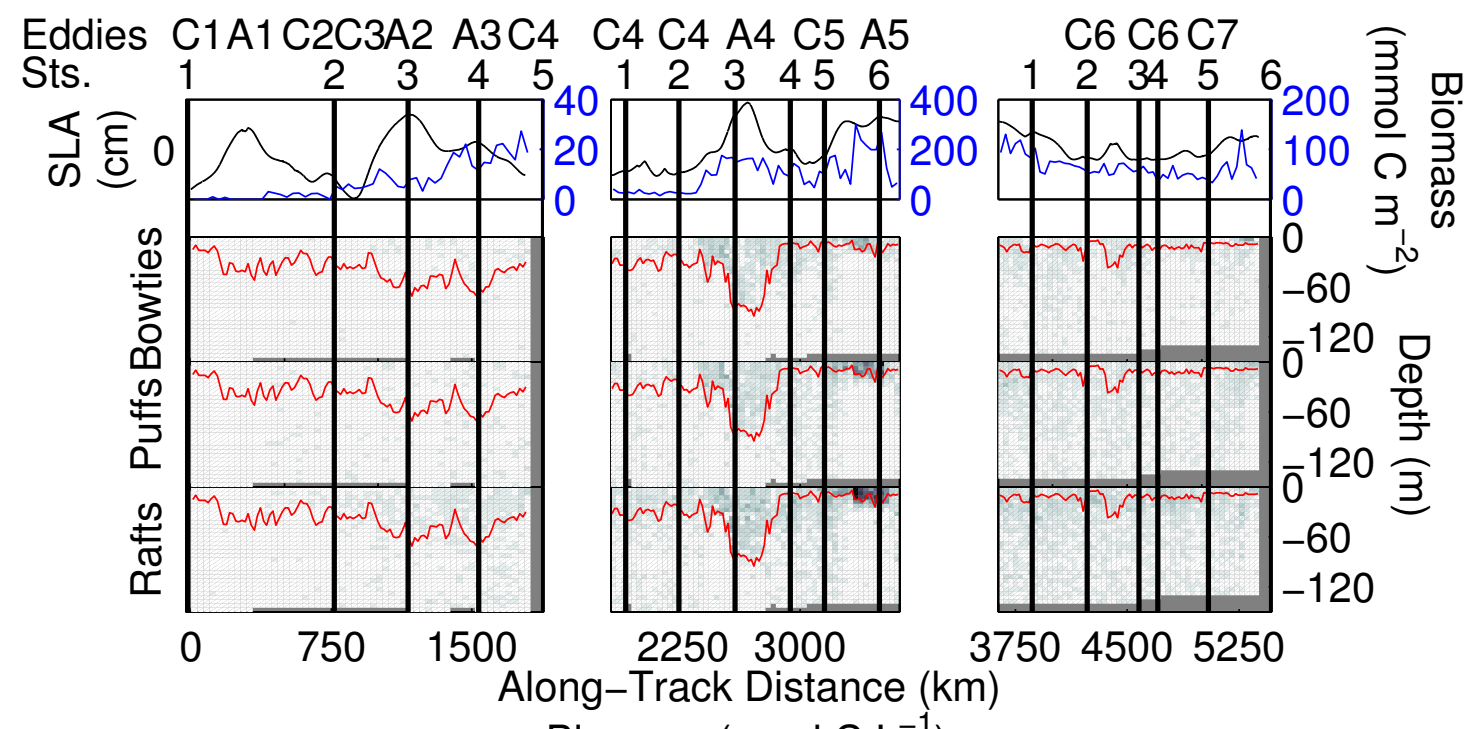

Biomass $\left(\mu \mathrm{mol} \mathrm{C} \mathrm{L}{ }^{-1}\right)$

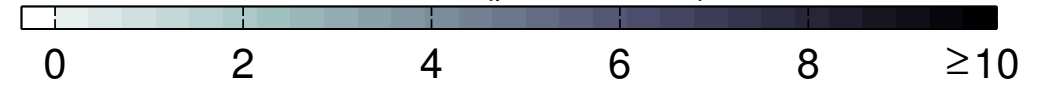

OC471 Section $1 \quad$ OC471 Section $2 \quad$ OC471 Section 3

Eddies C1A1 C2C3A2 A3C4 C4 C4 A4 C5 A5 C6 C6 C7
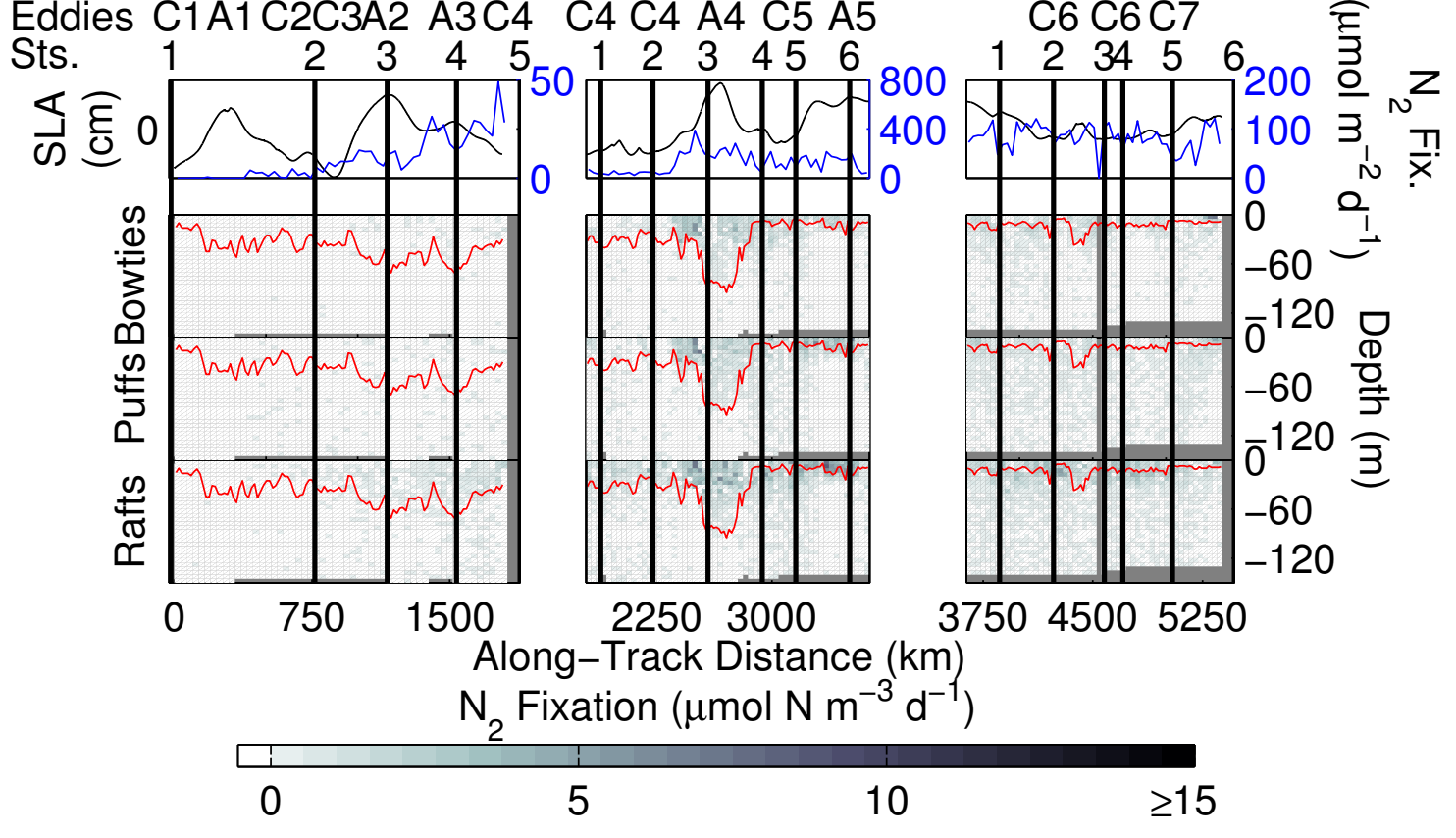
Figure 4-15: OC471 Trich biomass (upper) and modeled nitrogen fixation estimates (lower). For each section, the top panel displays sea level anomaly (black) and depthintegrated biomass/nitrogen fixation (blue). The lower three panels show biomass or nitrogen fixation by colony morphology. Black vertical lines mark station locations. Red lines designate mixed layer depth. Gray areas are regions not sampled by VPR.
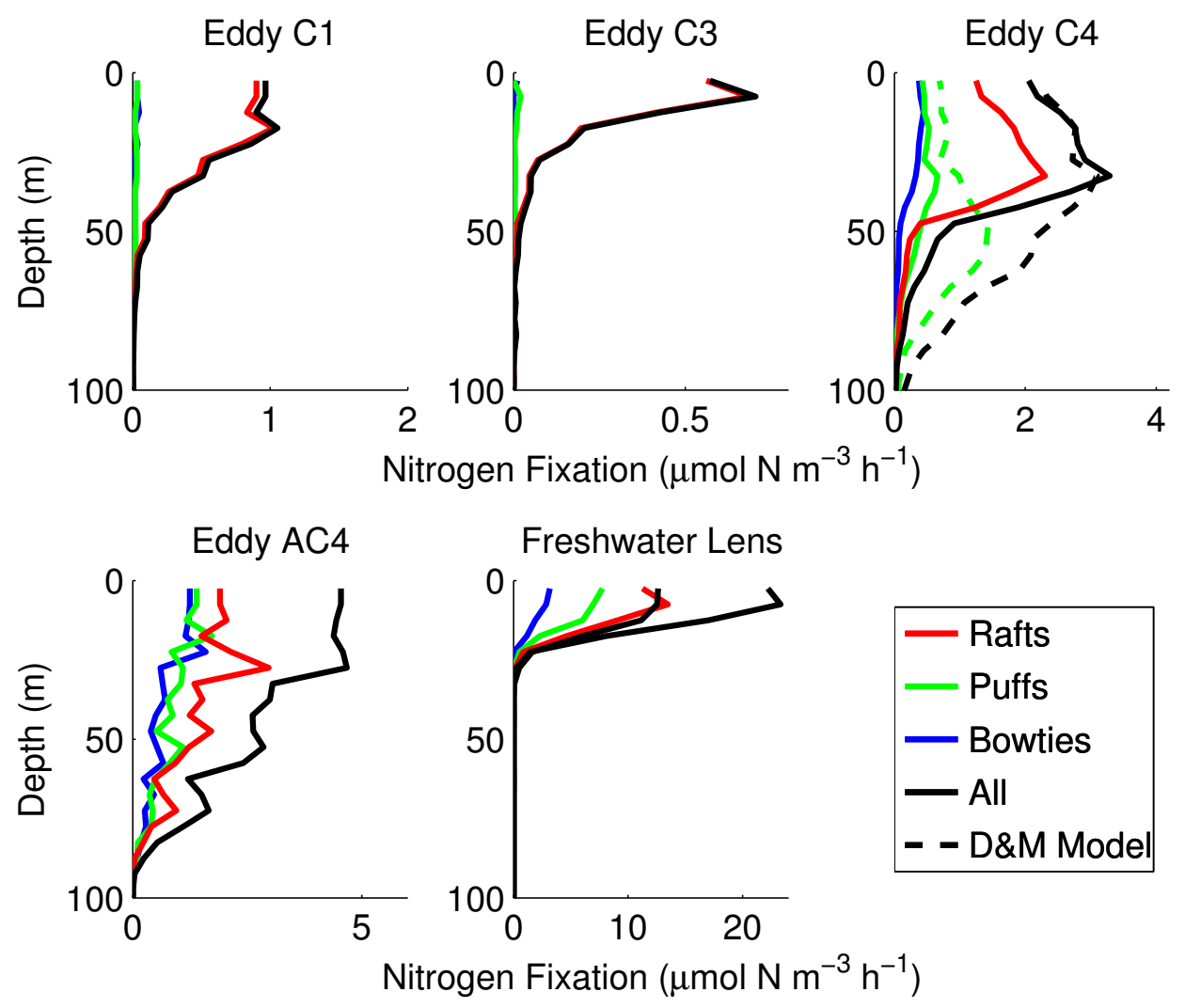

Figure 4-16: Nitrogen fixation versus depth by colony morphology for regions with varied physical characteristics from the two cruises. Upper three panels are from OC469 and lower two panels are from OC471. Dashed lines in upper right plot represent the application of the Davis and McGillicuddy (2006) model (multiplied by a factor of 0.16) to OC469 eddy C4 abundance profiles. The greater nitrogen fixation response at low light levels in that model produces a deeper subsurface peak in puff nitrogen fixation. 
(Subramaniam et al., 2008; Coles et al., 2004), seems to be a driver of geographical patterns in Trichodesmium abundance and nitrogen fixation.

Some differences are apparent between the VPR-based abundance and nitrogen fixation profiles and corresponding mean profiles from the Luo et al. (2012) database. The most significant is that, except in the southernmost region, the conventional mean surface abundances are lower than the VPR estimates. In one region, the western area from Davis and McGillicuddy (2006), the difference in the surface value exceeds the $95 \%$ confidence intervals on the Luo et al. (2012) mean. Subsurface maxima are present in the conventional mean vertical abundance profiles for all four regions analyzed (Figure 4-18; northern, northwestern, mid, and southern). These subsurface maxima, present in the 10-40 $\mathrm{m}$ depth bin, are absent from the corresponding mean VPR-based abundance profiles from Davis and McGillicuddy (2006) and the present study, although similar profiles were observed within smaller patches at some locations. The Luo et al. (2012) dataset includes single-depth surface measurements as well as full profiles. These additional surface profiles tend to skew the surface mean abundance slightly lower; however, the subsurface maximum appears even when calculations are restricted to stations with measurements at two or more depths ("profiles only" in Figure 4-18).

This inconsistency might be attributable to a difference in vertical location of the surface sample, since the VPR normally approaches no closer than within $5 \mathrm{~m}$ of the surface, whereas net or bottle samples may be taken in the upper one to two meters. Based on microscopic enumeration of colonies from bottle samples, ten out of 15 stations have lower abundances in the surface bottle sample than in one of the deeper samples from the same station from OC469. However, for OC471, the same was true for only one out of eight stations. Since VPR sampling on OC471 was more extensive than on OC469, OC471 contributes more to the mean profiles. Therefore, the difference in surface VPR-based abundances may simply reflect the conditions present during OC471. In fact, the profiles based on the Luo et al. (2012) data corresponding to seasonal and geographic ranges more closely tied to VPR sampling patterns (Fig- 
ure 4-13) do not exhibit the subsurface maximum. This suggests that this subsurface maximum may be more commonly observed at other times of year, possibly related to shifts in dominance from the surface-dwelling rafts typically observed in this study to the often more uniformly distributed puff morphology.

Some differences are not surprising considering the limited and different geographical and temporal coverage of both datasets. Notwithstanding these deviations, broad geographical trends are consistent across measurement techniques. Just as Davis and McGillicuddy (2006) observed higher abundances in the western portion of their VPR transect, profiles based on data west of $50^{\circ} \mathrm{W}$ in the $29-40^{\circ} \mathrm{N}$ range of the Luo et al. (2012) data are elevated compared to data from the full extent of the North Atlantic at those latitudes. Across the regions analyzed (Figure 4-18), abundances increase progressively to the south, with the mid region elevated compared to the northwestern region and the southern region higher still.

Based on the conventionally-derived profiles, the lower abundances in the northern region are more uniform with depth than the southern profiles, consistent with VPR observations by Davis and McGillicuddy (2006). Surface and deep confidence intervals overlap in the northern region to a greater extent than in the mid and southern region. However, even in the northern region, greatest variability and average abundance occurs in the upper $40 \mathrm{~m}$. Both the VPR-based estimates and the newly available compilation of traditionally sampled data (Luo et al., 2012) demonstrate greater abundance and nitrogen fixation rates compared to the conventional estimates reproduced in Davis and McGillicuddy (2006). Through analysis of the extensive Luo et al. (2012) data we see that the relatively high abundance and inferred nitrogen fixation at depth described by Davis and McGillicuddy (2006) may be attributable to geographical variability rather than underestimation of deep abundances by conventional sampling methods.

Mean nitrogen fixation profiles based on Luo et al. (2012) are slightly elevated in the 10-40 $\mathrm{m}$ range relative to the surface in the northern, northwestern and mid regions, but these subsurface peaks are less pronounced than in the abundance data 
(Figure 4-18). Bio-optical VPR nitrogen fixation estimates tend to be higher than the conventionally sampled estimates, particularly at the surface. Modeled and conventional nitrogen fixation profiles agree most closely in the southern region, where the VPR profiles fall within the $90 \%$ confidence intervals of the mean Luo et al. (2012) profiles at all depths. As in abundance, there is a southward increase in both amplitude and depth of nitrogen fixation in both the conventionally sampled and bio-optically modeled profiles. Mean rates in the 40-60 $\mathrm{m}$ range from the southern profile are nearly $40 \%$ of surface levels and are higher than mean surface rates in the northern profile. Deeper rates, at 60-100 m depth, are approximately an order of magnitude lower than in the 40-60 m range, contributing only a small fraction of depth-integrated nitrogen fixation.

The present bio-optical model of nitrogen fixation model tends to estimate higher rates near the surface and lower rates at depth compared to the adapted Davis and McGillicuddy (2006) model. This tendency can be explained by comparing the nitrogen-fixation-light response of the two models under mean midday conditions (Figure 4-19). The Davis and McGillicuddy (2006) model approaches its asymptotic nitrogen fixation value more quickly. Moving southward, the new model estimates increase relative to the adapted Davis and McGillicuddy (2006) estimates due to the response of the model to increasing surface irradiance. Surface irradiance does not impact the Davis and McGillicuddy (2006) model, in which nitrogen fixation is a function of the fraction of irradiance relative to the surface. In the southern region, the two models agree at depth and diverge near the surface where the adapted Davis and McGillicuddy (2006) model is low relative to the Luo et al. (2012) estimate and the OC469-OC471 estimate is high.

Application of the model for nitrogen fixation presented in this study to the Davis and McGillicuddy (2006) colony abundance profile for the wider northern region (Figure 4-18 bottom left panel) yields an integrated nitrogen fixation rate of $2.8 \mu \mathrm{mol} \mathrm{N} \mathrm{m}{ }^{-2} \mathrm{~d}^{-1}$, much lower than the $16.74 \mu \mathrm{mol} \mathrm{N} \mathrm{m}{ }^{-2} \mathrm{~d}^{-1}$ estimated by Davis and McGillicuddy (2006). However, modification of their estimate by the factor of 0.16 for 
consistency of surface nitrogen fixation rates with those measured in the present study reduces the Davis and McGillicuddy (2006) estimate to $2.7 \mu \mathrm{mol} \mathrm{N} \mathrm{m}{ }^{-2} \mathrm{~d}^{-1}$, nearly identical to the OC469-OC471 model estimate. The integrated nitrogen fixation based on profiles available in Luo et al. (2012) is slightly lower at $1.1 \mu \mathrm{mol} \mathrm{N} \mathrm{m} \mathrm{d}^{-1}$ with upper and lower bounds of approximately 0.16 and $2.7 \mu \mathrm{mol} \mathrm{N} \mathrm{m} \mathrm{N}^{-2} \mathrm{~d}^{-1}$ based on $95 \%$ confidence intervals in each depth bin. The difference of a factor of about 6 between the daily surface nitrogen fixation rates (based on diver-collected samples) used by Davis and McGillicuddy (2006) and those estimated in the present study is roughly consistent with the factor of 4 difference in nitrogen fixation reported by Orcutt et al. (2001) between diver- and net-collected samples, with some of the additional difference likely due to spatial and temporal variability. Additional methodological biases could contribute to variability in nitrogen fixation estimates. For instance, whereas the current study includes only nitrogen fixation measurements based on acetylene reduction assays, the Luo et al. (2012) database also includes nitrogen fixation measurements from ${ }^{15} \mathrm{~N}_{2}$ incubations. Mohr et al. (2010) reported that nitrogen fixation may be underestimated by the ${ }^{15} \mathrm{~N}_{2}$ incubation method due to failure of injected ${ }^{15} \mathrm{~N}_{2}$ gas bubbles to reach equilibrium with the water. The degree of underestimation depends on the incubation duration as the ${ }^{15} \mathrm{~N}_{2}$ bubble equilibrates with the water over time. However, Luo et al. (2012) report that nitrogen fixation rates from both methods distributed over a similar range. This apparent consistency between methods was verified for each of the four regions in which mean profiles were examined (Appendix A). Additional uncertainty in nitrogen fixation estimates made by acetylene reduction assay stems from from the choice of conversion ratio between acetylene reduction and nitrogen fixation. A ratio of 3 was used in the present study, whereas Luo et al. (2012) report ratios in the range of 3-4.

Similarly, modifying the Davis and McGillicuddy (2006) estimate for nitrogen fixation in the northwest subtropical Atlantic of $50.54 \mu \mathrm{mol} \mathrm{N} \mathrm{m} \mathrm{d}^{-1}$ by the factor of 0.16 produces an estimate of $8.1 \mu_{\mathrm{mol} \mathrm{N} \mathrm{m}}^{-2} \mathrm{~d}^{-1}$, only slightly lower than the OC469OC471 model estimate of $11.2 \mu \mathrm{mol} \mathrm{N} \mathrm{m}{ }^{-2} \mathrm{~d}^{-1}$. In that region, the estimate based on 
Luo et al. (2012) is 1.7 with lower and upper bounds of 0.04 and $4.0 \mu \mathrm{mol} \mathrm{N} \mathrm{m}{ }^{-2} \mathrm{~d}^{-1}$. There, as well as in the mid region, comprising the northern area sampled on OC469 and OC471 (Figure 4-18), modeled nitrogen fixation estimates based on the VPR are high compared to mean profiles from the Luo et al. (2012) data. Since the abundance estimates in these regions are not inconsistent with Luo et al. (2012), considering the level of variability in the data, it seems likely that this discrepancy is due to spatial and temporal variability in per-carbon nitrogen fixation rates and the limited spatial and temporal coverage of both the Luo et al. (2012) and VPR data.

Integrated nitrogen fixation for the southern region (Figure 4-18 bottom right panel) based on the VPR survey mean profile is estimated at $107 \mu \mathrm{mol} \mathrm{N} \mathrm{m} \mathrm{N}^{-2} \mathrm{~d}^{-1}$, or $80 \mu \mathrm{mol} \mathrm{N} \mathrm{m} \mathrm{d}^{-2}$ using the adjusted Davis and McGillicuddy (2006) model. The integrated nitrogen fixation rate based on the mean profiles from the Luo et al. (2012) data in this region is $145 \mu \mathrm{mol} \mathrm{N} \mathrm{m} \mathrm{N}^{-2} \mathrm{~d}^{-1}$, with upper and lower bounds of 64 and 228 $\mu \mathrm{mol} \mathrm{N} \mathrm{m} \mathrm{N}^{-1}$. All of these estimates fall in the lower end of the range of areal nitrogen fixation rates reported by Capone et al. (2005) in the southwestern tropical North Atlantic, reflecting the relatively low nitrogen fixation rates measured on these cruises. 
a) Depth-Integrated Trichodesmium Abundance (colonies $\mathrm{m}^{-2}$ )
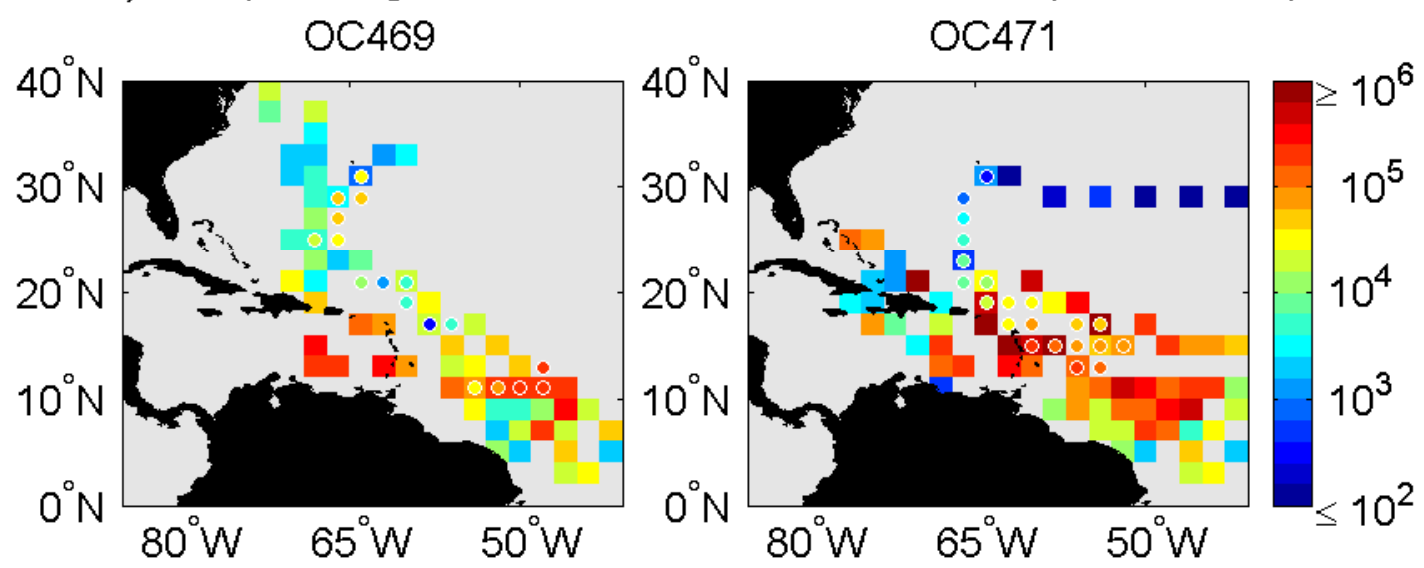

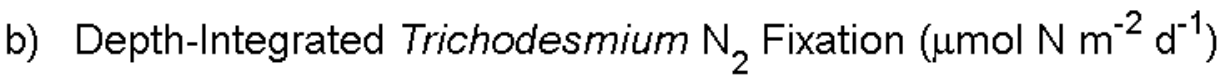
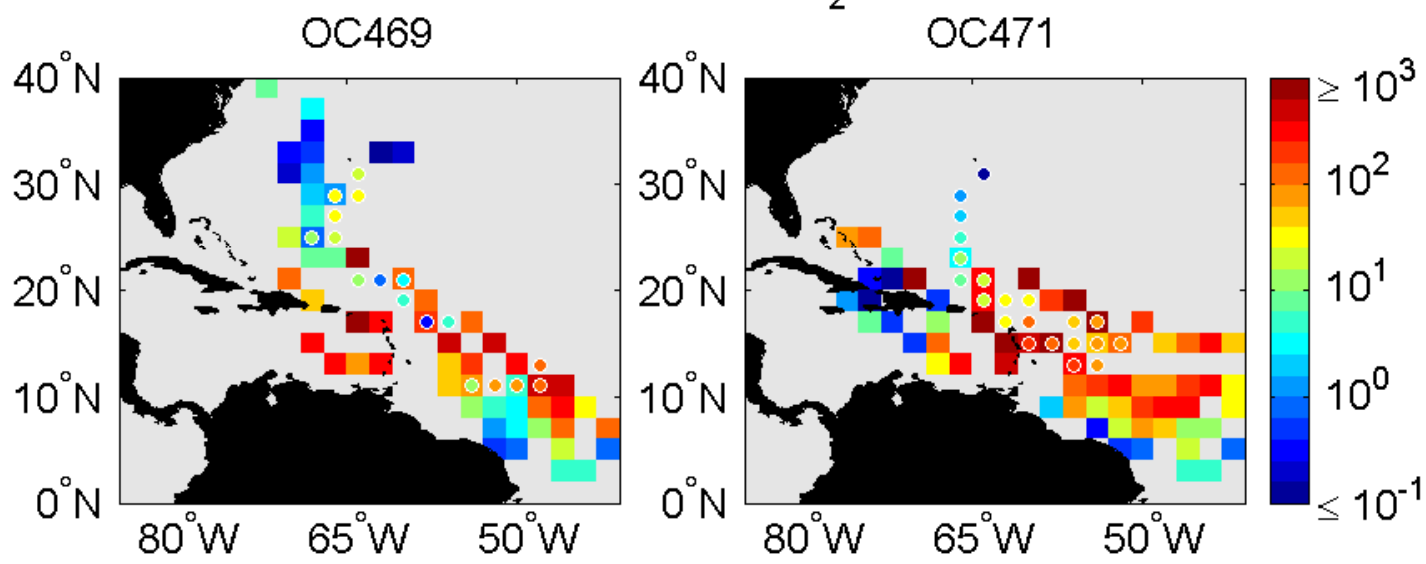

$\square$ Luo et al. (2012)

- VPR Estimate

Figure 4-17: Depth-integrated Trichodesmium a) colony abundance and b) nitrogen fixation. Circles represent VPR abundances or nitrogen fixation estimates with OC469 in the left panels and OC471 in the right panels. Squares represent data from the Luo et al. (2012) database within 90 year-days of the VPR data. All data are binned to two-degree resolution. Color mapping is by a log scale. 





Figure 4-18: Mean depth profiles of Trichodesmium abundance (upper) and Trichodesmium nitrogen fixation (lower) for several regions in the North Atlantic. The profiles are based on data from Luo et al. (2012) (squares) with 95\% confidence intervals (circles) and VPR transects. Estimates in dark blue are based on all Luo et al. (2012) data in each bin while only stations with measurements at two or more depths were included in the profile calculations (cyan). The geographical distribution of data from the Luo et al. (2012) database for each region is shown on an inset map in blue. From left to right, the regions are: northern $\left(75-8^{\circ} \mathrm{W}, 28-40^{\circ} \mathrm{N}\right)$; northwestern $(75-$ $\left.50^{\circ} \mathrm{W}, 29-40^{\circ} \mathrm{N}\right)$; mid $\left(75-50^{\circ} \mathrm{W}, 20-32^{\circ} \mathrm{N}\right)$; and southern $\left(65-50^{\circ} \mathrm{W}, 10-20^{\circ} \mathrm{N}\right)$. Also shown are nitrogen fixation rates estimated from the VPR abundances and the nitrogen fixation model developed here (magenta) as well as the adapted (multiplied by a factor of 0.16) version of the nonlinear model presented in Davis and McGillicuddy (2006) (green). In order to apply the new model to the Davis and McGillicuddy (2006) abundance profiles, the following values were assumed: surface irradiance of $38 \mu$ mol quanta $\mathrm{m}^{-2} \mathrm{~d}^{-1}$ based on mission composite SeaWiFS PAR for the region, vertical extinction coefficient of $0.05 \mathrm{~m}^{-1}$, mixed layer depth of $30 \mathrm{~m}$, and mean carbon per colony for puffs and rafts estimated from OC469 data, which was collected at nearly the same time of year as the Davis and McGillicuddy (2006) survey. In the northern and northwestern regions, the models were applied to mean abundance profiles, but in the mid and southern regions they were applied at all locations before taking the means. 


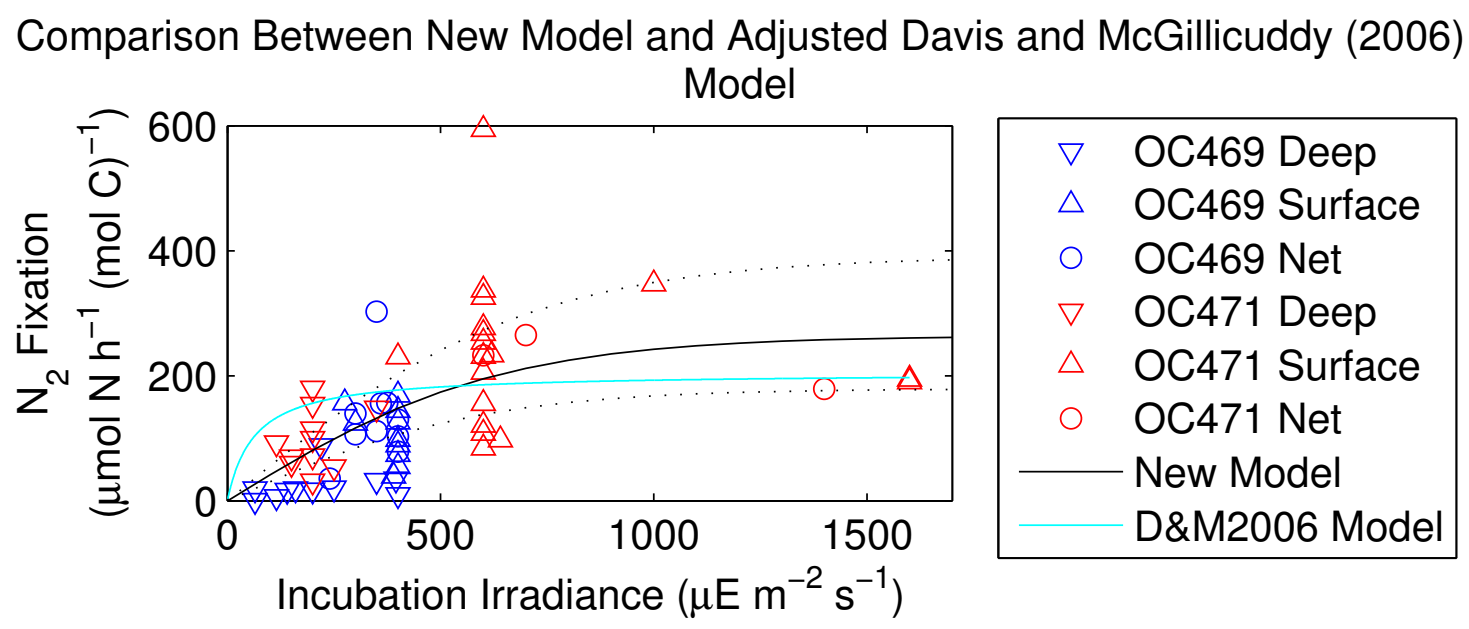

Figure 4-19: Comparison between response to light in the model developed for this study and the adapted version of the Davis and McGillicuddy (2006) model. The two model curves are overlaid on the nitrogen fixation-irradiance data used to derive the new model. In order to present the adapted Davis and McGillicuddy (2006) model in these axes, we converted from per-colony to per-carbon nitrogen fixation by dividing by the overall mean (puffs and rafts from both cruises) carbon to colony ratio of $6.182 \mathrm{e}-7 \mathrm{~mol} \mathrm{C}$ (colony) ${ }^{-1}$ and assumed a midday surface light intensity of $1600 \mu \mathrm{mol}$ quanta $\mathrm{m}^{-2} \mathrm{~s}^{-1}$.

\subsection{Conclusions}

Means and large-scale spatial trends in VPR-observed Trichodesmium colony distributions are not inconsistent with prior observations from the Luo et al. (2012) database. These results therefore do not provide evidence that conventional sampling methods have underestimated deep Trichodesmium colony populations due to disruption of deep colonies during sampling. However, the comparison cannot prove that there is no depth-bias in net-based sampling relative to VPR sampling of Trichodesmium colonies. First, the two methods were not applied to the same samples; we are only able to compare observations taken at different times in the same broad geographic areas. Second, the Luo et al. (2012) database that is the source of the "conventional" measurements does not specify in what manner each of the samples was collected, although it is clear that some are from net tows and others are from 
bottle casts. It is therefore not clear what fraction of the data comprising the conventional set are based on bottle samples, which may disrupt colonies less than net tows. Nonetheless, agreement with the conventionally sampled data in the Luo et al. (2012) database promotes confidence in the accuracy of the newer imaging-based VPR method.

The high-resolution sampling made possible by the VPR reveals covariation of Trichodesmium colony abundances with characteristics of the physical environment. Colony abundances are consistently higher within the mixed layer, suggesting that physical mixing is of primary importance in determining the depth-distribution of colonies. Colony distributions also showed relationships with the eddy field, with elevated abundances colocated with cyclones at three locations on the fall cruise and with two anticyclones, both with anomalously low salinities, on the spring cruise.

Colony abundances and depth distributions varied by morphology. Rafts were most abundant overall, and consistently peaked in abundance close to the surface. Puffs were the next most abundant and tended to have greater relative abundance deeper in the water column compared to rafts. Puff abundances tended to covary geographically with raft abundances except for a local maximum of puff colony abundance in a single location below the mixed layer in OC469 cyclone C4 and an increase in relative abundance at the southern portion of OC471. Bowties were the least abundant morphology and had distributions similar to puffs and rafts.

Nitrogen fixation was estimated along VPR transects using a simple bio-optical model relating per-carbon nitrogen fixation to irradiance. Several assumptions and approximations were made in the application of the model. Biomass estimates were made assuming a uniform carbon content per colony for puffs and rafts for each cruise. Local variations in colony size could therefore lead to error in the biomass estimate which would propagate to the nitrogen fixation calculation. Because rafts were most abundant, they were more heavily represented in nitrogen fixation experiments. Puffs such as those below the mixed layer in OC469 cyclone C4 may be adapted to thrive in different conditions and therefore exhibit different nitrogen fixation rates and re- 
sponse of nitrogen fixation to irradiance. This could lead to error, particularly in the morphology-specific nitrogen fixation rates. Additionally, the model itself cannot explain all the observed variability in per- carbon nitrogen fixation rates. It cannot reproduce variability in per-carbon nitrogen fixation due to factors other than irradiance, such as iron or phosphorus stress. Furthermore, nitrogen fixation measurements are susceptible to systematic error due to sampling methods, including underestimation common in ${ }^{15} \mathrm{~N}_{2}$ incubation methods (Mohr et al., 2010) and varying ratios used to convert acetylene reduction rates to nitrogen fixation estimates.

Within this model framework, two factors control the depth-distribution of Trichodesmium nitrogen fixation in the southwestern North Atlantic. The first is light, which decays with depth due to exponential attenuation. The second factor is the vertical distribution of Trichodesmium biomass, which is in turn controlled to a significant extent by stratification. Thus, PAR and stratification were the significant drivers of local variations in the depth of modeled nitrogen fixation. Further modeling studies could shed light on the mechanisms by which stratification affects the depth of nitrogen fixation by testing whether the relationship can be explained purely by physical mixing of colonies deeper in the water column or if the effects of mixing on nutrient supply, of iron or phosphorus, for example, may also contribute.

Colony morphology may also be a significant factor in determining the depth of nitrogen fixation, as deep puff aggregations such as that observed during OC469 contribute to deep nitrogen fixation. This effect could potentially be larger (or smaller) than estimated here if the response of nitrogen fixation to light in deep puff populations is different from that modeled. Future studies could include nitrogen fixation experiments on puff colonies collected from a similar deep aggregation. Perhaps genetic studies of the Trichodesmium clade distribution in the vicinity of the puff aggregation could shed some light on its origin.

This VPR-based study did not find deep nitrogen fixation in the tropical North Atlantic at the upper range of levels hypothesized by Davis and McGillicuddy (2006) based on nitrogen fixation rates in diver-collected samples. However, nitrogen fixation 
rates were here modeled based on rates from net-collected samples, which Davis and McGillicuddy (2006) suggested could be low due to mechanical disturbance of the colonies during sampling, based on comparisons of rates from net- and diver-collected samples by Orcutt et al. (2001). Additionally, nitrogen fixation rates may be systematically underestimated due to other aspects of standard measurement practices, such as gas injection in ${ }^{15} \mathrm{~N}_{2}$ techniques (Mohr et al., 2010). Therefore it seems more likely that any underestimation of nitrogen fixation rates by Trichodesmium may be due to deficiencies in standard techniques for the measurement of nitrogen fixation than to underestimation of abundance. However, this study did not directly compare simultaneous VPR and net sampling of colony abundance, and therefore bias in sampling by net tows cannot be ruled out definitively.

Compared to the northern region sampled by Davis and McGillicuddy (2006), nitrogen fixation profiles from the tropical Atlantic exhibited higher rates. In mean profiles encompassing all VPR sections from OC469 and OC471, 25\% of colonies were below $50 \mathrm{~m}$ depth, but these colonies contribute only $7 \%$ of total bio-optically estimated nitrogen fixation. Mean nitrogen fixation and abundance profiles were not inconsistent with mean profiles calculated from the Luo et al. (2012) dataset based on the confidence intervals. However, the Luo et al. (2012) mean profiles revealed abundance and nitrogen fixation levels of the comparable to those at the surface as deep as $50 \mathrm{~m}$. The pattern of nearly uniform Trichodesmium puff colony abundance with depth throughout the upper 120 m described by Davis and McGillicuddy (2006) may be more typical of low-abundance regions sampled in that study. The VPR observations from the present study demonstrate high abundance deep Trichodesmium populations only in specific and infrequent circumstances, consistently associated with anomalously deep mixed layers. Further study could use the VPR combined with net tows to specifically target patches of deeply mixed colonies and measure their nitrogen fixation rates to determine whether patterns associated with mean conditions apply there. 


\section{Chapter 5}

\section{Conclusions}

\subsection{Overview}

This thesis contains findings from two research cruises in the western subtropicaltropical North Atlantic conducted during fall 2010 and spring 2011. The primary data set used in these analyses was sampled using the Video Plankton Recorder (VPR), a towed underwater microscope. Other sources of data, including nitrogen fixation experiments, CTD casts, underway data, and satellite altimetry have been used to augment the analysis. This research was motivated by the question, what physical and biogeochemical factors influence the magnitude and distribution of Trichodesmium abundance and nitrogen fixation in the western North Atlantic?

Chapter 2 outlines a procedure for the extraction of abundance estimates from VPR data in situations where the taxa of interest is of low relative abundance and the size of the dataset large. By subsampling the original set of images at a lower sampling rate than that of the original data, a smaller set can be constructed of a size suitable for manual classification verification. The resulting data is consistent with the original in the mean, but additional noise is introduced at small scales. In order to reduce the effects of noise, the data can be analyzed on a coarser grid. In this way, reduced sampling rate results in reduced spatial resolution. However, this approach allows useful information to be extracted from otherwise intractable datasets. 
Chapter 3 discusses mesoscale patterns in Trichodesmium distribution observed using the VPR in fall 2010 and spring 2011. Abundances were locally elevated in cyclonic compared to anticyclonic eddies in a relatively low abundance region transected during fall 2010. Simulations of the evolution of a buoyant tracer field in the presence of cyclonic and anticyclonic eddies supported the hypothesis that wind-driven motions in eddies could lead to increased buoyant tracer concentration at the center of cyclones and decreased concentration at the center of anticyclones. However, the simulations did not reproduce the full magnitude of concentration difference present in the observations, suggesting that additional factors contributed to the observed distributions.

In spring 2011, abundance was elevated in anticyclones relative to cyclones in the southern part of the study area. This was attributed to riverine influence, likely a source of phosphorus and iron required for Trichodesmium growth. Anticyclones form in the North Brazil Current region and drift northwest, carrying Amazon plume water. In this way, the reach of the Amazon river influence is extended from the river mouth into the tropical North Atlantic.

Chapter 4 describes a bio-optical model for nitrogen fixation based on rates measured on the two cruises. The model is applied to the VPR tracks, combining abundance data with modeled nitrogen fixation rates. Vertical and geographical patterns in nitrogen fixation and abundance are compared with estimates from the database compiled by Luo et al. (2012) and found to be not inconsistent. Therefore, the findings do not support the hypothesis that net tows under-sample fragile deep colony populations observable by VPR. Whereas Davis and McGillicuddy (2006) reported differences between depth distributions of colonies sampled by VPR with those sampled by traditional methods, comparison of the same VPR data with local observations from the more extensive database of Luo et al. (2012) does not reveal inconsistency. However, the Luo et al. (2012) database contains bottle-sampled abundance data and therefore may not represent typical profiles based on net tows. Estimated nitrogen fixation rates at depth are also not inconsistent with previous measurements through- 
out the regions analyzed. In the high-abundance southern region of the study area, mean nitrogen fixation rates from the Luo et al. (2012) data as deep as $50 \mathrm{~m}$ are approximately $40 \%$ of mean surface values.

\section{$5.2 \quad$ Future Directions}

Some observations made though this work raise further questions that could be addressed through future research. Here we outline a few of these questions and possible paths for future investigation.

Methodologically, it would be useful to track down the source of the deviation between abundance measured by VPR and microscopic enumeration that was encountered at high abundance levels. This could be investigated further through statistical analysis of Trichodesmium population structure at small scales and the effects of different sampling procedures on abundance predictions. Also, it would be useful, if possible, to record the frame rate and number of dropped images during future VPR surveys. This would allow researchers to definitively determine whether bias in abundance estimates correlates with dropped images. Finally, statistical methods could be used to develop theory identifying the optimal subsampling rate in cases where the full dataset is too large to analyze.

Next we describe some of the scientific questions raised through analysis of the VPR observations. One of the distinctive features observed during the fall VPR survey was a region in which there appeared to be depth-segregation by colony morphology, with rafts peaking at the surface and puffs near the base of the mixed layer. This region was sampled with the VPR, but the nearest station was just outside its bounds. With VPR data only, questions regarding potential physiological differences between the two groups remain unanswered. For instance, do these apparently segregated populations of puffs and rafts fix nitrogen at the same rate? Are the populations genetically distinct? Are they experiencing different levels of nutrient stress? If a similar region should be identified on a future cruise, we recommend samples be 
taken and nitrogen fixation experiments performed at the location of both the puff and raft abundance maxima. It would also be interesting to follow such a region over time in order to see if the vertical distributions of the two populations evolved. The VPR is useful in this type of targeted work because it can provide information on population structure in realtime while the ship is underway.

Additionally, there are a number of related questions regarding colony morphology. Is morphology genetically determined or shaped by environmental conditions? Is there niche differentiation in Trichodesmium populations associated with colony morphology? Does vertical migration play a role? Future research in the laboratory and through genetic analysis of field samples could provide answers.

Through idealized eddy simulations, we showed that over a period of several months, wind-driven motions could produce convergence in cyclones and dilution in anticyclones. Further investigation using a comprehensive ecosystem and biogeochemical model could elucidate conditions under which the eddy/wind mechanism might produce observable patterns. Specifically, what growth conditions are required for physical aggregation to emerge as a dominant driver of colony distributions?

Further observational work could provide additional evidence for the relationship we have inferred between Trichodesmium and anticyclones containing river plume water. In this study, Trichodesmium was observed in anticyclones originating near the North Brazil Current. In other studies, elevated iron and phosphorus have been encountered in similar anticyclones. Observation of such an anticyclone and its chemical and ecological characteristics from the time of its development through the end of a Trichodesmium bloom would allow more complete evaluation of the connection. Comparative genetic analyses of Trichodesmium populations within fresh water associated with the Amazon river and in the open ocean could indicate whether these populations are distinct. This information could be used to evaluate the hypothesis that the fresh water lens originating in the Amazon plume acts as a "nursery", seeding regional Trichodesmium populations.

Future modeling work could elucidate the physical controls on the depth distribu- 
tion of Trichodesmium. It would be interesting to investigate vertical profiles induced under different buoyancy and wind-driven mixing conditions. This line of study could shed light on the related questions, what factors control carbon export associated with Trichodesmium nitrogen fixation, and what are the pathways by which this export occurs?

Finally, this work highlights the importance of further evaluation and refinement of methods used to estimate nitrogen fixation rates in Trichodesmium. Absence of disagreement between the VPR-based findings in this study and canonical net-based estimates of the depth-distribution of colony abundance in the southwestern North Atlantic suggests that if differences are present, they are not as large as the recorded spatial and temporal variability. Thus, if nitrogen fixation by Trichodesmium colonies is methodologically underestimated, the underestimation likely stems from another source. A culprit may be found in current methods used for measuring nitrogen fixation, as indicated by Mohr et al. (2010). Further investigation leading to improved accuracy in nitrogen fixation measurements could significantly further all areas of research involving rates of diazotrophic activity.

\subsection{Summary and Scientific Impact}

The primary scientific contributions of this thesis are in three areas. The first is the development and verification of a procedure for extracting abundance of relatively small populations from VPR data with high numbers of images. The second is the identification of two mechanisms by which physical drivers shape Trichodesmium distributions at the mesocale. The third is the comparison of VPR and canonical net-sampled Trichodesmium abundances in the high-abundance southwestern North Atlantic. Comparison between VPR and conventional abundance measurements in the Luo et al. (2012) database does not provide evidence of deep populations that are missing from the conventional data.

The VPR is a powerful tool for investigating mesoscale and even submesoscale 
patterns in abundance. As we have shown, with human-mediated identification, the VPR can be successfully used to estimate abundances even when the target organism represents a small fraction of total images. The co-located physical and abundance measurements provided by the VPR allow for investigation of connections between abundance and the physical environment at smaller spatial scales than can be achieved through water sampling with Niskin bottles. After successfully developing an analysis approach balancing accuracy with processing time, we employed this dataset to analyze relationships between Trichodesmium and the physical environment at the mesoscale.

Several relationships between Trichodesmium and the mesoscale eddy field have been identified in other studies. Davis and McGillicuddy (2006) observed higher Trichodesmium abundances in anticyclones in the North Atlantic subtropical gyre in fall 2003. This was later explained by down-gradient transport of biomass and phosphorus in anticyclonic eddies originating in the Gulf Stream (McGillicuddy, 2014). In the North Pacific Subtropical Gyre, Guidi et al. (2012) observed elevated Trichodesmium abundance in a frontal region associated with horizontal stirring and surface convergence.

Here, we identified two additional mechanisms. First, based on patterns observed in fall 2010, we uncovered a physical interaction between wind-driven surface motions in eddies and buoyant colonies. This mechanism produces convergence and increased abundance in cyclones and divergence and decreased abundance in anticyclones and may manifest during periods of steady growth. The second pattern, observed in spring 2011, involved increased abundance in the presence of anomalously fresh water. This freshwater originated in the Amazon River plume and may have been transported northwestward in part by translation of anticyclonic eddies formed in the vicinity of the North Brazil current. It seems likely that these eddies also transport nutritional substrates necessary for Trichodesmium growth such as iron and phosphorus. The timescales of these mechanisms are very different; patterns due to the physical aggregation mechanism emerge over a span of several months, whereas nutrient-driven 
growth is expected to occur on timescales of a few days typical of Trichodesmium doubling times. Thus, we expect patterns driven by spatial gradients in growth and mortality to dominate when those gradients are present, as might be expected under bloom conditions. However, in the absence of such gradients, the effects of physical aggregation due to wind-driven motions in eddies could emerge.

In addition to these mesoscale patterns, VPR data were useful in assessing vertical distributions of colony abundance. With addition of a bioptical model, these estimates were extended to nitrogen fixation. Estimates of nitrogen fixation were roughly consistent with estimates made in the same region and seasons using traditional sampling methods, indicating consistency between the VPR approach and more established protocols. These findings demonstrate several ways the VPR can be an effective addition to a sampling scheme, producing valuable results at scales difficult to obtain by most other methods.

We have concluded that "missing nitrogen" is unlikely to be attributable to under sampling of deep colony populations. We have also shown that the mesoscale eddy field can produce mesoscale variability in Trichodesmium abundance. This variability may be produced through multiple mechanisms, which are expected to dominate under different conditions and in different geographical locations. Thus, the primary questions motivating this research have been successfully answered. In addition, a number of intriguing observations have prompted further questions regarding the physical and biological factors influencing the abundance and activity of Trichodesmium colonies. These questions may lead to avenues of fruitful future research and discovery. 
Appendix A

Nitrogen Fixation Method

Comparison 


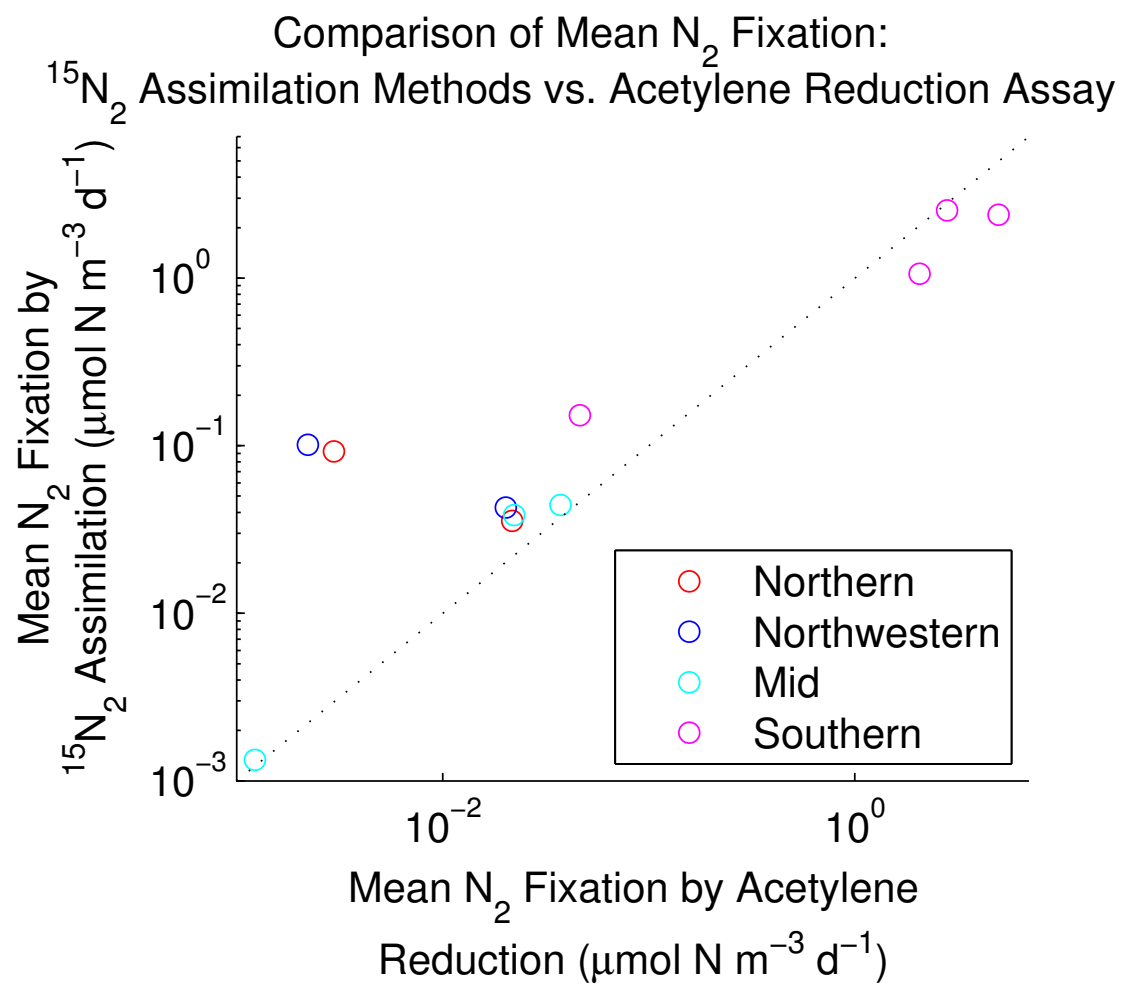

Figure A-1: Comparison between ${ }^{15} \mathrm{~N}_{2}$ incubation and acetylene reduction assay nitrogen fixation methods based on data included in the Luo et al. (2012) database. Values presented are means for each of the depths and the four geographical regions displayed in Figure 4-18. 


\section{Bibliography}

Andresen, E., J. Lohscheider, E. Šetlikova, I. Adamska, M. Šimek, and H. Küpper, 2010: Acclimation of Trichodesmium erythraeum ISM101 to high and low irradiance analysed on the physiological, biophysical and biochemical level. New Phytologist, 185, 173-188.

Batchelor, G. K., 1967: An Introduction to Fluid Dynamics. 2000th ed., Cambridge University Press.

Bergquist, B. A. and E. A. Boyle, 2006: Iron isotopes in the Amazon River system: Weathering and transport signatures. Earth and Planetary Science Letters, 248, 54-68, doi:10.1016/j.epsl.2006.05.004.

Berman-Frank, I., K. D. Bidle, L. Haramaty, and P. G. Falkowski, 2004: The demise of the marine cyanobacterium, Trichodesmium spp., via an autocatalyzed cell death pathway. Limnology and Oceanography, 49 (4), 997-1005.

Berman-Frank, I., P. Lundgren, Y.-B. Chen, H. Küpper, Z. S. Kolber, B. Bergman, and P. Falkowski, 2001: Segregation of nitrogen fixation and oxygenic photosynthesis in the marine cyanobacterium Trichodesmium. Science, 294, 1534-1537.

Berman-Frank, I., A. Quigg, Z. V. Finkel, A. J. Irwin, and L. Haramaty, 2007: Nitrogen-fixation strategies and Fe requirements in cyanobacteria. Limnology and Oceanography, 52, 2260-2269.

Breitbarth, E., A. Oschlies, and J. LaRoche, 2007: Physiological constraints on the global distribution of Trichodesmium - effect of temperature on diazotrophy. Biogeosciences, 4, 53-61.

Breitbarth, E., J. Wohlers, J. Kläs, J. LaRoche, and I. Peeken, 2008: Nitrogen fixation and growth rates of Trichodesmium IMS-101 as a function of light intensity. Marine Ecology Progress Series, 359, 25-36.

Brock, T. D., 1981: Calculating solar radiation for ecological studies. Ecol. Modeling, 14, 1-19.

Capone, D. G., J. A. Burns, J. P. Montoya, A. Subramaniam, C. Mahaffey, T. Gunderson, A. F. Michaels, and E. J. Carpenter, 2005: Nitrogen fixation by Trichodesmium 
spp.: An important source of new nitrogen to the tropical and subtropical North Atlantic Ocean. Global Biogeochemical Cycles, 19.

Capone, D. G. and J. P. Montoya, 2001: Nitrogen fixation and denitrification. Marine Microbiology, J. H. Paul, Ed., Academic Press, Methods in Microbiology, Vol. 30, 501 - 515, doi:http://dx.doi.org/10.1016/S0580-9517(01)30060-0, URL http://www.sciencedirect.com/science/article/pii/S0580951701300600.

Capone, D. G., J. P. Zehr, H. W. Paerl, B. Bergman, and E. J. Carpenter, 1997: Trichodesmium, a globally significant marine cyanobacterium. Science, 276, 12211229.

Carpenter, E. J., J. M. O’Neil, R. Dawson, D. G. Capone, P. J. A. Siddiqui, T. Roenneberg, and B. Bergman, 1993: The tropical diazotrophic phytoplankter Trichodesmium: biological characteristics of two common species. Marine Ecology Progress Series, 95, 295-304.

Carpenter, E. J., A. Subramaniam, and D. G. Capone, 2004: Biomass and primary productivity of the cyanobacterium Trichodesmium spp. in the tropical N Atlantic ocean. Deep-Sea Research I, 51, 173-203.

Chen, Y.-B., B. Dominic, S. Zani, M. T. Mellon, and J. P. Zehr, 1999: Expression of photosynthesis genes in relation to nitrogen fixation in the diazotrophic filamentous nonheterocystous cyanobacterium Trichodesmium sp. ims 101. Plant Molecular Biology, 41, 89-104.

Chen, Y.-B., J. P. Zehr, and M. Mellon, 1996: Growth and nitrogen fixation ofthe diazotrophic filamentous nonheterocystous cyanobacterium Trichodesmium sp. IMS 101 in defined media: Evidence for a circadian rhythm. J. Phycol., 32, 916-923.

Chen, Y.-L. L., S.-H. Tuo, and H.-Y. Chen, 2011: Co-occurrence and transfer of fixed nitrogen from Trichodesmium spp. to diatoms in the low-latitude Kuroshio Current in the NW Pacific. Marine Ecology Progress Series, 421, 25-38.

Codispoti, L. A., J. A. Brandes, J. P. Christensen, A. H. Devol, S. W. A. Naqvi, H. W. Paerl, and T. Yoshinari, 2001: The oceanic fixed nitrogen and nitrous oxide budgets: Moving targets as we enter the anthropocene? Scientia Marina, 65 (Suppl. 2), 85-105.

Coles, V. J., M. T. Brooks, J. Hopkins, M. R. Stukel, P. L. Yager, and R. R. Hood, 2013: The pathways and properties of the amazon river plume in the tropical north atlantic ocean. Journal of Geophysical Research: Oceans, 118, 6894-6913.

Coles, V. J., C. Wilson, and R. R. Hood, 2004: Remote sensing of new production fueled by nitrogen fixation. Geophysical Research Letters, 31, L06 301, doi: 10.1029/2003GL019018. 
Collares-Pereira, M. and A. Rabl, 1979: The average distribution of solar-radiation correlations between diffuse and hemispherical, and between daily and hourly instolation values. Sol. Energy, 22, 155-168.

Davis, C. S. and D. J. McGillicuddy, 2006: Transatlantic abundance of the $\mathrm{N}_{2}$-fixing colonial cyanobacterium Trichodesmium. Science, 312 (5779), 1517 - 1520, URL http://dx.doi.org/10.1126/science.1123570.

Davis, C. S., D. J. McGillicuddy, V. K. Kosnyrev, C. M. Petrik, N. C. Loomis, and J. A. Eaton, 2013: Distribution of Trichodesmium and other plankton in relation to physical properties across the Caribbean Sea determined by in situ optical imaging, Submitted, Marine Ecology Progress Series.

Davis, C. S., F. T. Thwaites, S. M. Gallager, and Q. Hu, 2005: A three-axis fast-tow digital video plankton recorder for rapid surveys of plankton taxa and hydrography. Limnology and Oceanography Methods, 3, 59-74.

Deutsch, C., J. L. Sarmiento, D. M. Sigman, N. Gruber, and J. P. Dunne, 2007: Spatial coupling of nitrogen inputs and losses in the ocean. Nature, 445, 163-167.

Dewar, W. K. and G. R. Flierl, 1987: Some effects of the wind on rings. Journal of Physical Oceanography, 17 (10), 1653-1667.

Dyhrman, S. T., P. D. Chappell, S. T. Haley, J. W. Moffett, E. D. Orchard, J. B. Waterbury, and E. A. Webb, 2006: Phosphonate utilization by the globally important marine diazotroph Trichodesmium. Nature, 439, 68-71.

Eberl, R. and E. J. Carpenter, 2007: Association of the copepod macrosetella gracilis with the cyanobacterium Trichodesmium spp. in the North Pacific Gyre. Marine Ecology Progress Series, 333, 205-212.

Fernández, A., B. Mouriño-Carballido, A. Bode, M. Varela, and E. Marañón, 2010: Latitudinal distribution of Trichodesmium spp. nad $\mathrm{n}_{2}$ fixation in the Atlantic Ocean. Biogeosciences, 7, 3167-3176.

Fitzsimmons, J. N., R. Zhang, and E. A. Boyle, 2013: Dissolved iron in the tropical North Atlantic Ocean. Marine Chemistry, 154, 87-99, doi: 10.1016/j.marchem.2013.05.009.

Fong, A. A., D. M. Karl, R. Lukas, R. M. Letelier, J. P. Zehr, and M. J. Church, 2008: Nitrogen fixation in an anticyclonic eddy in the oligotrophic North Pacific Ocean. The ISME Journal, 2, 663-676.

Foster, R. A., A. Subramaniam, and J. P. Zehr, 2009: Distribution and activity of diazotrophs in the Eastern Equatorial Atlantic. Environmental Microbiology, 11 (4), 741-750. 
Franks, P. J. S., 1992: Sink or swim: accumulation of biomass at fronts. Marine Ecology Progress Series, 82 (1), 1-12.

Fratantoni, D. M. and P. L. Richardson, 2006: The evolution and demise of North Brazil Current rings. Journal of Physical Oceanography, 36, 1241-1264.

Galloway, J. N., et al., 2004: Nitrogen cycles: past, present, and future. Biogeochemistry, 70, 153-226.

Goebel, N. L., C. A. Edwards, M. J. Church, and J. P. Zehr, 2007: Modeled contributions of three types of diazotrophs to nitrogen fixation at Station ALOHA. The ISME Journal, 1, 606-619.

Goebel, N. L., et al., 2010: Abundance and distribution of major groups of diazotrophic cyanobacteria and their potential contribution to $\mathrm{N}_{2}$ fixation in the tropical Atlantic Ocean. Environmental Microbiology, 12 (12), 3272-3289.

Gruber, N., 2004: The dynamics of the marine nitrogen cycle and its influence on atmospheric co2. The ocean carbon cycle and climate, M. Follows and T. Oguz, Eds., Kluwer Academic, Dordrecht, NATO ASI Series, 97-148.

Gruber, N., 2008: The marine nitrogen cycle: Overview and challenges. Nitrogen in the marine environment, D. G. Capone, D. A. Bronk, M. R. Mulholland, and E. J. Carpenter, Eds., Amsterdam, The Netherlands: Elsevier, 1-50.

Guidi, L., et al., 2012: Does eddy-eddy interaction control surface phytoplankton distribution and carbon export in the North Pacific Subtropical Gyre? Journal of Geophysical Research, 117, G02 024, doi:10.1029/2012JG001984.

Hawser, S. P., J. M. O’Neil, M. R. Roman, and G. A. Codd, 1992: Toxicity of blooms of the cyanobacterium Trichodesmium to zooplankton. Journal of Applied Phycology, 4, 79-86.

Heithoff, A., 2011: $\mathrm{N}_{2}$ fixation by subsurface populations of Trichodesmium: an important source of new nitrogen to the North Atlantic Ocean. MS Thesis, Department of Biology, Massachusetts Institute of Technology and the Woods Hole Oceanographic Institution.

Hellweger, F. L. and A. L. Gordon, 2002: Tracing Amazon River water into the Carribbean Sea. Journal of Marine Research, 60, 537-549.

Hewson, I., S. R. Govil, D. G. Capone, E. J. Carpenter, and J. A. Fuhrman, 2004: Evidence of Trichodesmium viral lysis and potential significance for biogeochemical cycling in the oligotrophic ocean. Aquati Microbial Ecology, 36, 1-8.

Hewson, I., R. S. Poretsky, S. T. Dyhrman, B. Zielinski, A. E. White, H. J. Tripp, J. P. Montoya, and J. P. Zehr, 2009: Microbial community gene expression within 
colonies of the diazotroph, Trichodesmiumns, from the Southwest Pacific Ocean. The ISME Journal, 3, 1286-1300.

Holl, C. M. and J. P. Montoya, 2008: Diazotrophic growth of the marine cyanobacterium Trichodesmium ims101 in continuous culture: effects of grwoth rate on $\mathrm{n}_{2}$-fixation rate, biomass, and c:n:p stoichiometry. J. Phycol., 44, 929-937.

Hood, R. R., A. Subramaniam, L. R. May, E. J. Carpenter, and D. G. Capone, 2002: Remote estimation of nitrogen fixation by Trichodesmium. Deep-Sea Research II, 49, $123-147$.

$\mathrm{Hu}$, Q. and C. Davis, 2006: Accurate automatic quantification of taxa-specific plankton abundance using dual classification with correction. Marine Ecology Progress Series, 306, 51-61.

Hynes, A. M., P. D. Chappell, S. T. Dyhrman, S. C. Doney, and E. A. Webb, 2009: Cross-basin comparison of phosphorus stress and nitrogen fixation in Trichodesmium. Limnology and Oceanography, 54 (4), 1438-1448.

Jassby, A. D. and T. Platt, 1975: Mathematical formulation of the relationship between photosynthesis and light for phytoplankton. Limnology and Oceanography, 21, 540-548.

Jenkins, W. and J. Goldman, 1985: Seasonal oxygen cycling and primary production in the Sargasso Sea. Journal of Marine Research, 43 (2), 465-491.

Kirk, J. T. O., 1994: Light and Photosynthesis in Aquatic Ecosystems. Cambridge University Press.

Ledwell, J. R., D. J. McGillicuddy, and L. A. Anderson, 2008: Nutrient flux into an intense deep chlorophyll layer in a mode-water eddy. Deep Sea Research Part II: Topical Studies in Oceanography, 55, 1139-1160, doi:10.1016/j.dsr2.2008.02.005.

Lenes, J. M. and C. A. Heil, 2010: A historical analysis of the potential nutrient supply from the $\mathrm{n}_{2}$ fixing marine cyanobacterium Trichodesmium spp. to Karenia brevis blooms in the eastern Gulf of Mexico. Journal of Plankton Research, 32 (10), $1421-1431$.

Letelier, R. M. and D. M. Karl, 1996: Role of Trichodesmium spp. in the productivity of the subtropical North Pacific Ocean. Marine Ecology Progress Series, 133, 263273.

Letelier, R. M. and D. M. Karl, 1998: Trichodesmium spp. physiology and nutrient fluxes in the North Pacific subtropical gyre. Aquati Microbial Ecology, 15, 265-276.

Luo, Y.-W., et al., 2012: Database of diazotrophs in global ocean: abundance, biomass and nitrogen fixation rates. Earth Syst. Sci. Data, 4, 47-73. 
Mahaffey, C., A. F. Michaels, and D. G. Capone, 2005: The conundrum of marine $\mathrm{N}_{2}$ fixation. American Journal of Science, 305, 546-595.

Mahaffey, C., R. G. Williams, G. A. Wolff, N. Mahowald, W. Anderson, and M. Woodward, 2003: Biogeochemical signatures of nitrogen fixation in the eastern North Atlantic. Geophysical Research Letters, 30 (6), 1300, doi:10.1029/2002GL016542.

Mahowald, N. M., et al., 2005: Atmospheric global dust cycle and iron inputs to the ocean. Global Biogeochemical Cycles, 19, GB4025, doi:10.1029/2004GB002402.

Martin, A. P. and K. J. Richards, 2001: Mechanisms for vertical nutrient transport within a North Atlantic mesoscale eddy. Deep-Sea Research II, 48, 757-773.

McGillicuddy, D. J., 2014: Do Trichodesmium spp. populations in the North Atlantic export most of the nitrogen they fix? Global Biogeochemical Cycles, 28, doi:10.1002/2013GB004652.

McGillicuddy Jr., D., et al., 2007: Eddy/wind interactions stimulate extraordinary mid-ocean plankton blooms. Science, 316, 1021-1026, doi:10.1126/science.1136256.

Mohr, W., T. Großkopf, D. W. R. Wallace, and J. LaRoche, 2010: Methodological underestimation of oceanic nitrogen fixation rates. PLoS ONE, 5 (9), e12 583, doi: 10.1371/journal.pone.0012583.

Monteiro, F. M., S. Dutkiewicz, and M. J. Follows, 2011: Biogeographical controls on the marine nitrogen fixers. Global Biogeochemical Cycles, 25, GB2003, doi:10.1029/2010GB003902.

Monteiro, F. M., M. J. Follows, and S. Dutkiewicz, 2010: Distribution of diverse nitrogen fixers in the global ocean. Global Biogeochemical Cycles, 24, GB3017, doi:10.1029/2009GB003731.

Mulholland, M. R., S. Floge, E. J. Carpenter, and D. G. Capone, 2002: Phosphorus dynamics in cultures and natural populations of Trichodesmium spp. Marine Ecology Progress Series, 239, 45-55.

Okubo, A., 1971: Oceanic diffusion diagrams. Deep-Sea Research, 18, 789-802.

O'Neil, J. M., 1998: The colonial cyanobacterium Trichodesmium as a physical and nutritional substrate for the harpacticoid copepod macrosetella gracilis. Journal of Plankton Research, 20 (1), 43-59.

Orchard, E. D., J. W. Ammerman, M. W. Lomas, and S. T. Dyhrman, 2010: Dissolved inorganic and organic phosphorus uptake in Trichodesmium and the microbial community: The importance of phosphorus ester in the Sargasso Sea. Limnology and Oceanography, 55 (3), 1390-1399. 
Orcutt, K. M., F. Lipschultz, K. Gundersen, R. Arimoto, A. F. Michaels, A. H. Knap, and J. R. Gallon, 2001: A seasonal study of the significance of $\mathrm{N}_{2}$ fixation by Trichodesmium spp. at the Bermuda Atlantic Time-series Study (BATS) site. Deep-Sea Research II, 48, 1583-1608.

Platt, T., C. L. Gallegos, and W. G. Harrison, 1980: Photoinhibition of photosynthesis in natural assemblages of marine phytoplankton. Journal of Marine Research, 38, 687-701.

Post, A. F., et al., 2002: Spatial and temporal distribution of Trichodesmium spp. in the stratified gulf of aqaba, red sea. Marine Ecology Progress Series, 239, 241-250.

Richardson, P. L. and G. Reverdin, 1987: Seasonal cycle of velocity in the atlantic north equatorial countercurrent as measured by surface drifters, current meters, and ship drifts. Journal of Geophysical Research, 92.

Rodier, M. and R. L. Borgne, 2010: Population and trophic dynamics of Trichodesmium thiebautii in the se lagoon of new caledonia. Comparison with t. erythraeum in the sw lagoon. Marine Pollution Bulletin, 61, 349-359.

Saino, T. and A. Hattori, 1978: Diel variation in nitrogen fixation by a marine bluegreen alga, Trichodesmium thiebautii. Deep-Sea Research, 25, 1259-1263.

Sañudo-Wilhelmy, S. A., et al., 2001: Phosphorus limitation of nitrogen fixation by Trichodesmium in the central Atlantic Ocean. Nature, 441, 66-69.

Smith, R. D., M. E. Maltrud, F. O. Bryan, and M. W. Hecht, 2000: Numerical simulation of the north atlantic ocean at 1/10 degree. Journal of Physical Oceanography, 30, 1532-1561.

Sohm, J. A. and D. G. Capone, 2010: Zonal differences in phosphorus pools, turnover and deficiency across the tropical North Atlantic Ocean. Global Biogeochemical Cycles, 24, GB2008, doi:10.1029/2008GB003414.

Sohm, J. A., C. Mahaffey, and D. G. Capone, 2008: Assessment of relative phosphorus limitation of Trichodesmium spp. in the North Pacific, North Atlantic, and the north coast of Australia. Limnology and Oceanography, 53 (6), 2495-2502.

Subramaniam, A., et al., 2008: Amazon River enhances diazotrophy and carbon sequestration in the tropical North Atlantic Ocean. PNAS, 105 (30), 10 460-10 465.

Taboada, F. G., R. G. Gil, J. Höfer, S. González, and R. Anadón, 2010: Trichodesmium spp. population structure in the eastern North Atlantic subtropical gyre. Deep-Sea Research I, 57, 65-77.

Tovar-Sanchez, A. and S. A. Sañudo-Wilhelmy, 2011: Influence of the amazon river on dissolved and intra-cellular metal concentrations in Trichodesmium colonies along 
the western boundary of the sub-tropical North Atlantic Ocean. Biogeosciences, 8, 217-225, doi:10.5194/bg-8-217-2011.

Tyrrell, T., E. Marañón, A. J. Poulton, A. R. Bowie, D. S. Harbour, and E. M. S. Woodward, 2003: Large-scale latitudinal distribution of Trichodesmium spp. in the Atlantic Ocean. Journal of Plankton Research, 25 (4), 405-416.

Villareal, T. A. and E. J. Carpenter, 2003: Buoyancy regulation and the potential for vertical migration in the oceanic cyanobacterium Trichodesmium. Microbial Ecology, 45 (1), pp. 1-10.

Walsby, A., 1992: The gas vesicles and buoyancy of Trichodesmium. Marine Pelagic Cyanobacteria: Trichodesmium and other Diazotrophs, E. Carpenter, D. Capone, and J. Rueter, Eds., Springer Netherlands, NATO ASI Series, Vol. 362, 141-161.

Walsby, A. E., 1978: The properties and buoyancy-providing role of gas vacuoles in Trichodesmium ehrenberg. Br. Phycol. J., 13, 103-116.

Ward, B. A., S. Dutkiewicz, C. M. Moore, and M. J. Follows, 2013: Iron, phosphorus, and nitrogen supply ratios define the biogeography of nitrogen fixation. Limnology and Oceanography, 58 (6), 2059-2075, doi:10.4319/lo.2013.58.6.2059.

Westberry, T., D. Siegel, and A. Subramaniam, 2005: An improved biooptical model for the remote sensing of Trichodesmium spp. blooms. Journal of Geophysical Research-Oceans, 110 (C6), doi:10.1029/2004JC002517, URL http://dx.doi.org/10.1029/2004JC002517\%7D.

Westberry, T. K. and D. A. Siegel, 2006: Spatial and temporal distribution of Trichodesmium blooms in the world's oceans. Global Biogeochemical Cycles, $20 \quad$ (4), doi:10.1029/2005GB002673, URL http://dx.doi.org/10.1029/2005GB002673\%7D.

White, A. E., Y. H. Spitz, and R. M. Letelier, 2006: Modeling carbohydrate ballasting by Trichodesmium spp. Marine Ecology Progress Series, 323, 35-45.

Wilson, S. T., Z. S. Kolber, S. Tazzi, J. P. Zehr, and D. M. Karl, 2012: Nitrogen fixation, hydrogen cycling, and electron transport kinetics in Trichodesmium erythraeum (cyanobacteria) strain IMS101. J. Phycol., 48, 595-606.

Zhong, Y., A. Bracco, and T. A. Villareal, 2012: Pattern formation at the ocean surface: Sargassum distribution and the role of the eddy field. Limnology 85 Oceanography: Fluids \& Environments, 2, 12-27. 